\title{
RNA-mediated Genomic Arrangements in Mammalian Cells
}

Sachin Kumar Gupta, Liming Luo, and Laising Yen*

Department of Pathology \& Immunology, Department of Molecular \& Cellular Biology, Dan L. Duncan Cancer Center, Baylor College of Medicine, Houston, TX 77030, USA

*Corresponding author and lead contact:

Laising Yen, Ph.D.

Associate Professor

Department of Pathology \& Immunology

Department of Molecular and Cellular Biology

Baylor College of Medicine,

Phone: +1-713-798-1180

Email: yen@bcm.edu

Key words:

Gene fusion, non-coding RNA, chimeric RNA, cancer, genomic rearrangement, transcriptional conflict, TMPRSS2-ERG, TMPRSS2-ETV1, AZI1, androgen

\section{Summary}

One of the hallmarks of cancer is the formation of oncogenic fusion genes as a result of chromosomal translocations. Fusion genes are presumed to occur prior to fusion RNA expression. However, studies have reported the presence of fusion RNAs in individuals who were negative for chromosomal translocations. These observations give rise to "the cart before the horse" hypothesis, in which fusion RNA precedes the fusion gene and guides the genomic rearrangements that ultimately result in gene fusions. Yet RNA-mediated genomic rearrangement in mammalian cells has never been demonstrated. Here we provide evidence that expression of a chimeric RNA drives formation of a specified gene fusion via genomic rearrangement in mammalian cells. The process is (1) specified by the sequence of chimeric RNA involved, (2) facilitated by physiological hormone levels, (3) permissible regardless of intra-chromosomal (TMPRSS2-ERG) or inter-chromosomal (TMPRSS2-ETV1) fusion, and (4) can occur in normal cells prior to malignant transformation. We demonstrate that, contrary to "the cart before the horse" model, it is the antisense rather than sense chimeric RNAs that effectively drive gene fusion, and that this disparity can be explained by transcriptional conflict. Furthermore, we identified an endogenous RNA AZI1 that acts as the 'initiator' RNA to induce TMPRSS2-ERG fusion. RNA-driven gene fusion demonstrated in this report provides important insight in early disease mechanism, and could have fundamental implications in the biology of mammalian genome stability, as well as gene editing technology via mechanisms native to mammalian cells. 


\section{Introduction}

Fusion genes are among the most cancer-specific molecular signatures known. They are important for understanding cancer mechanisms and developing useful clinical biomarkers and anti-cancer therapies (Mitelman et al., 2007). Fusion gene formation as a result of chromosomal translocations is presumed to occur prior to fusion RNA expression. However, several studies have reported the presence of fusion transcripts in individuals without detectable fusion genes at the genomic DNA level. For instance, the AML1-ETO fusion transcript, associated with a subtype of acute myeloid leukemia, was present in some patients who were negative for chromosomal translocations (Langabeer et al., 1997). Other fusion RNAs, such as $B C R-A B L$, $M L L-A F 4, T E L-A M L 1, P M L-R A R \alpha$, and NPM-ALK, were reported in healthy individuals (Janz et al., 2003). Although the discrepancy between the presence of fusion transcripts and the absence of fusion genes could result from detection limitations of the methodologies employed, fusion transcripts in normal cells could also arise from RNA trans-splicing in the absence of chromosomal translocations (Zaphiropoulos, 2011). Indeed, JAZF1-JJAZ1 fusion transcripts are expressed in normal human endometrial tissue and an endometrial cell line in the absence of chromosomal translocation ( $\mathrm{Li}$ et al., 2008). Furthermore, trans-splicing between JAZF1 and JJAZ1 was demonstrated to occur in vitro using cellular extracts, resulting in a fusion RNA similar to that transcribed from the JAZF1-JJAZ1 fusion gene in endometrial stromal sarcomas (Li et al., 2008). These observations raise the possibility that cellular fusion RNAs created by trans-splicing act as guide RNAs to mediate genomic rearrangements. A precedent for RNAmediated genomic arrangements is found in lower organisms such as ciliates (Fang and Landweber, 2012; Nowacki et al., 2008). Rowley and Blumenthal (Rowley and Blumenthal, 2008) coined this as "the cart before the horse" hypothesis, in that "RNA before DNA" defies the normal order of the central dogma of biology: DNA $\rightarrow$ RNA $\rightarrow$ protein (Crick, 1970). Despite important implications in biology and human cancer, RNA-mediated genomic rearrangement in mammalian cells has not been directly demonstrated. In this report, we provide the first evidence that expression of a specific chimeric RNA can lead to specified gene fusion in mammalian cells.

\section{$\underline{\text { Results }}$}

To test whether the expression of a fusion RNA in mammalian cells can lead to a specific gene fusion, the TMPRSS2-ERG fusion (Perner et al., 2006; Tomlins et al., 2005), found in 50\% of prostate cancers, was selected as a model. Both the TMPRSS2 and ERG genes are located on chromosome 21, an intra-chromosomal configuration prone to rearrangements. To recapitulate TMPRSS2-ERG fusion gene formation, we used the LNCaP prostate cancer cell line that lacks the TMPRSS2-ERG fusion (Horoszewicz et al., 1980; Tomlins et al., 2005). Furthermore, treating LNCaP cells with androgen increases the chromosomal proximity between the TMPRSS2 and ERG genes (Bastus et al., 2010; Lin et al., 2009; Mani et al., 2009), which was thought to increase the possibility of gene fusion. To test "the cart before the horse" hypothesis 
(Rowley and Blumenthal, 2008; Zaphiropoulos, 2011), we transiently expressed a short fusion RNA consisting of two exons, TMPRSS2 exon-1 joined to ERG exon-4, which is a short fragment of a full-length TMPRSS2-ERG fusion RNA that is most common in prostate cancer (Fig. 1A, upper panel). This short fusion RNA mirrors the presumptive trans-spliced fusion RNA product that is generated only in the sense orientation because the correct splice sites are absent in the antisense orientation. However, because the 'antisense' sequence should, in theory, contain the same template information for guiding genomic rearrangements, we tested both the sense and antisense short fusion RNA. Each was individually expressed using either a CMV or a U6 promoter (Fig. 1A, upper panel) and designated as 'input RNA' to distinguish them from the 'endogenous' full-length fusion RNA transcribed from the genome.

We transiently transfected LNCaP cells with either plasmid and treated the cells with dihydrotestosterone (DHT, a metabolite of testosterone) for 3 days. If the expression of an input RNA leads to a TMPRSS2-ERG gene fusion, it is expected that the endogenous full-length fusion RNAs would be transcribed from the newly induced fusion gene. Specific RT-PCR assays were designed to distingwish between endogenous full-length fusion RNAs and the input RNAs exogenously expressed from the plasmids (see Fig. S1A for primer designs). As shown in Fig. $1 \mathrm{~A}$, expression of the sense short fusion RNA resembling the trans-spliced product, either by the CMV or U6 promoter (Fig. 1A lower panel, lane 1 and 2 respectively), led to no detection of an induced endogenous fusion transcript. Expression of a longer version of sense fusion RNA consisting of four exons (TMPRSS2 exon-1 joined to ERG exon-4/5/6) also failed to induce the endogenous fusion transcript (Fig. S2). In contrast, expression of antisense short fusion RNAs induced a clear band of 721 bp (Fig. 1A lower panel, lane 3 and 4). Sanger sequencing revealed that the induced band contains TMPRSS2 exon-1 fused to ERG exons-4/5/6/7 (Fig. S3), and that the exons are joined by annotated splice sites, which would be expected of mature endogenous fusion mRNA derived from the TMPRSS2-ERG fusion gene. This induced fusion transcripts cannot possibly arise from the sequence of input RNAs as the expression plasmids used contain only TMPRSS2 exon- 1 and ERG exon-4 without the ERG exon-5/6/7 sequence. Notably, the induction was more pronounced when the antisense input RNA was driven by the U6 promoter (Fig. 1A lower panel, antisense-2, lane 4) compared to the CMV promoter (antisense-1, lane 3). These differences (antisense vs. sense, U6 vs. CMV) are not caused by differing amounts of input RNA because all input RNAs were expressed at relatively equal levels (Fig. 1A, lower panel). Transfection with a parental plasmid containing mCherry sequence (Fig. 1A, lane 5), DHT treatment without plasmid transfection (Fig. 1A, lane 6), and PCR reaction without cDNA served as RT-PCR controls (Fig. 1A, lane 8), all resulted in no endogenous fusion transcript. In addition, all experiments were performed independently at least four times and results were identical. Together, the data suggest that expression of an input RNA with chimeric sequence can lead to the induction of a specified endogenous fusion transcript in human cells. Surprisingly, the antisense, rather than the sense version of input RNA, exhibits the capacity of induction. 
Antisense input RNAs described above contain 218 nt against the entire ERG exon-4 and $78 \mathrm{nt}$ against the entire TMPRSS2 exon-1 (Fig. 1A), suggesting that $78 \mathrm{nt}$ is sufficient to specify a parental gene for gene fusion. Furthermore, because the effective input RNAs are of the 'antisense' orientation, the data imply that the input RNAs may not require an RNA junction resembling that of the TMPRSS2-ERG fusion transcript generated by splicing in the sense orientation. To further analyze the sequence requirement, we used the U6 promoter to express a series of antisense input RNAs with 75 nt complementary to ERG exon-4 joined to various segments (33, 52, 67, $82 \mathrm{nt}$ ) that are complementary to TMPRSS2 near the exon-1/intron-1 boundary (Fig. 1B). A parallel set of sense input RNAs were also tested as controls (Fig. 1C). As shown in Fig. 1B (lower panel), all antisense RNAs, with the exception of antisense-3, induced fusion transcripts even though their target regions span the exon/intron boundary. The level of induction peaked for antisense-5, which contains 52 nt designed to anneal with TMPRSS2, suggesting that this number of nucleotides might be optimal to specify a parental gene for induction. The results were confirmed using a different, but more efficient, primer pair (Fig. 1B, lower panel; primer design in Fig. S1B) followed by Sanger sequencing of the induced band (Fig. S4). In contrast to the antisense input RNA, all corresponding sense input RNAs failed to induce endogenous fusion transcripts (Fig. 1C, lower panel). This was true even when the sense input RNA was intentionally expressed at a much higher level than the antisense RNA (Fig. S5). Additional experiments using plasmids with a severed U6 promoter (Fig. S6), to eliminate input RNA expression, also confirmed that it is the antisense input RNAs expressed from plasmids, not the DNA sequence of plasmids, that induce the observed TMPRSS2-ERG fusion transcripts.

As shown in Fig. 1D, the amount of endogenous fusion transcript induced by antisense-5 (the most effective antisense input RNA) appears to correlate with the concentration of DHT used, presumably because the hormone increases the chromosomal proximity between the TMPRSS2 and ERG genes (Bastus et al., 2010; Lin et al., 2009; Mani et al., 2009). Antisense-5 was effective at DHT concentrations as low as $20 \mathrm{nM}$ as revealed by sensitive nested PCR (Fig. 1D lane 3), indicating that fusion events induced by input RNA can occur under physiologically relevant androgen conditions (Boyce et al., 2004). As a control, DHT treatment alone up to $2 \mu \mathrm{M}$ failed to induce fusion (Fig. S7). Titration of DHT showed that the induction by antisense-5 reaches the $50 \%$ of maximal level $\left(\mathrm{EC}_{50}\right)$ at $0.9 \mu \mathrm{M}$ DHT (Fig. S7). Under this standard $\mathrm{EC}_{50}$ condition, we estimated that the percentage of LNCaP cells induced by antisense- 5 to express the TMPRSS2-ERG fusion transcript is approximately 1 in $10^{3}$ or $10^{4}$ cells (see assay in Fig. S8). Together, these results demonstrated that the induction by input RNA can occur at physiologically relevant hormone levels, but does not represent a high frequency event.

Although induced fusion is infrequent, all antisense RNAs described in Fig. 1B successfully induced endogenous fusion RNA except antisense-3, which is only $30 \mathrm{nt}$ longer than antisense-5 in the arm targeting TMPRSS2 intron-1 (Fig. 1B, upper panel). To test whether its inability to induce was due to input RNA length or the specific target sequence in TMPRSS2 intron-1, we constructed a hybrid antisense (antisense-7) that shifted the 52 nt recognition window of 
antisense-5 to target the TMPRSS2 intron-1 region covered by antisense-3 (Fig. 1E). This alteration resulted in the loss of induction (Fig. 1E, lane 1 vs. 3), implying that the inability of antisense-3 to induce is not reflective of input RNA length. Rather its targeting arm may interfere with a motif important for the fusion process. BLAST alignment of the genomic DNA sequence revealed an imperfect stem (named stem A) potentially formed by the sense genomic TMPRSS2 sequence complementary to the sense genomic ERG sequence (Fig. 2A, left). We reasoned that this genomic DNA stem ( $\mathrm{Tm}=\sim 44^{\circ} \mathrm{C}$ ) could potentially stabilize a three-way junction that involves an RNA/DNA duplex formed by the antisense-5 RNA and its targeted genomic DNA in a sequence-specific manner (Fig. 2A, left). If correct, then the formation of this putative threeway junction would be disrupted by antisense-3 because its recognition sequence invades the genomic DNA stem (Fig. 2A, left). Consistent with the idea that induction requires bringing TMPRSS2 and ERG gene in close proximity, expression of antisense-5 as two separate halves (Fig. 2A right panel, antisense-5A and -5B) severed the link between TMPRSS2 (52 nt) and ERG (75 nt) sequence within the input RNA, resulting in the loss of induction (Fig. 2B, lane 1 to 3 ).

To test whether the proposed three-way junction formation could facilitate induction, we used BLAST alignment to identify several intron locations where genomic DNA stems could be formed by the sense genomic TMPRSS2 sequence paired with the sense genomic ERG sequence (stems B to G in Fig. 2C and 2D; genomic coordinates in Fig. S9; sequences flanking the stems in Fig. S10). Matching antisense input RNAs (termed antisense-B1 to G1) were then designed to facilitate the formation of a three-way junction with the possible intron stems (Fig. 2D) that would mirror the three-way junction formed by antisense-5 on stem A as postulated in Fig 2A. Because these input RNAs target the introns (Fig. 2C) and contain no exon sequence, any observed induction of endogenous fusion transcripts composed of exons cannot arise from the sequence of input RNAs or plasmids used to express them. As shown in Fig. 2E, targeting genomic DNA stem B, C, and D that exhibit higher DNA stem stability $\left(\mathrm{Tm}=40^{\circ} \mathrm{C}, 40^{\circ} \mathrm{C}\right.$, and $44^{\circ} \mathrm{C}$, respectively) by the corresponding antisense input RNAs clearly induced fusion transcripts (Fig. 2E, lanes 2 to 4). In contrast, targeting less a stable stem $\mathrm{E}, \mathrm{F}$, and $\mathrm{G}\left(\mathrm{Tm}=30^{\circ} \mathrm{C}, 24^{\circ} \mathrm{C}\right.$, and $16^{\circ} \mathrm{C}$, respectively) failed to induce fusion transcripts (Fig. 2E, lanes 5 to 7). To disrupt the three-way junction invovling stem B, C, and D, six additional antisense RNAs (antisense-B2, B3, C2, C3, D2, and D3) were designed with one side of their recognition sequence altered to invade each of the respective genomic DNA stems on the TMPRSS2 side or the ERG side (Fig. S11). These modifications were chosen to mirror the interference on stem A by antisense-3. Similar antisense RNAs were also designed to invade stem A (Fig. S12). In all cases, invasion of the genomic DNA stems by the modified input RNAs resulted in the complete loss of induction (Fig. $2 \mathrm{~F}$ ). While these results by no means necessitate that a three-way junction is required for fusion transcript induction, they nevertheless suggest that such transiently stabilized structures may 'facilitate' the process and could have important implications in developing gene editing technologies via mechanisms native to mammalian cells. Consistent with earlier observations, the corresponding sense version of the effective antisense input RNAs (sense-B1, C1, D1) all failed to induce fusion transcripts (Fig. 2G, lanes 2 to 4 ). 
The fact that antisense input RNAs, but not their sense counterparts, induce fusion transcripts, raises the possibility that the former act as a docking station to mediate trans-splicing between endogenous sense TMPRSS2 and ERG pre-mRNAs. Because that the antisense, but not the sense input RNAs, are complimentary to both sense TMPRSS2 and ERG pre-mRNAs, they can basepare with both parental pre-mRNAs, thus resulting in spliced fusion transcripts without the requirement of genomic rearrangement. This mechanism, however, is unlikely as the major contributor to the observed induction for the following reasons. First, although TMPRSS2 is expressed in LNCaP cells (Fig. 3A, upper panel), endogenous ERG mRNA is not detected in LNCaP cells (Tomlins et al., 2005) in the presence or absence of DHT or before and after transfection of antisense-5 (Fig. 3A, middle and lower panel with different primer pairs). In fact, parental ERG mRNA was not detected even using three rounds of nested RT-PCR using various primer sets (Fig. S13). Therefore prior to and during induction, no or an insufficient number of parental ERG mRNAs are available in LNCaP cells as raw material for trans-splicing to account for the level of induced fusion transcript. Second, after initial transient transfection and DHT treatment for 3 days, we continued to propagate and enrich the induced LNCaP population for 52 days in the absence of DHT (experimental procedures described in Fig. S14). As shown in the lower panel of Fig. 3B, antisense-5 RNA transiently expressed by plasmids was degraded and completely absent beyond day 17. In contrast, the induced fusion transcript was continuously expressed and enriched up to day 52 in the absence of antisense input RNA and DHT (Fig. 3B upper panel), indicating the persistent nature of the induced fusion product. Taken together, these results strongly suggest that the induced expression of the TMPRSS2-ERG fusion transcript is the consequence of gene fusion at the DNA level, which has a permanent nature. This is in contrast to the result of induced trans-splicing at the RNA level mediated by antisense input RNA, which is transient and requires the continuous presence of input RNAs.

To provide definite evidence of gene fusion via genomic rearrangement, we used genomic PCR to identify the genomic breakpoint induced by antisense- 5 in the enriched LNCaP population (primer designs in Fig. S15A and Fig. 3C). As shown in Fig. 3D, the un-rearranged wildtype TMPRSS2 and ERG alleles were amplified by gene-specific primer pair A/B and C/D both in untransfected cells (Fig. 3D, lane 1 and 2) and enriched LNCaP cells (Fig. 3D, lane 4 and 5). In contrast, a genomic fusion band of $\sim 862$ bp amplified by fusion-specific primer pair A/D was present only in the enriched LNCaP population (Fig. 3D, lane 6) and absent in untransfected LNCaP cells (lane 3). Sanger sequencing of the excised fusion band (Fig. 3D, lane 6) revealed the exact genomic breakpoint located within TMPRSS2 intron-1 (chr21:41502038, GRCh38/hg38) and ERG intron-3 (chr21:38501207, GRCh38/hg38) (Fig. 3E; full-length Sanger sequence shown in S16). Intriguingly, within TMPRSS2 intron-1 the induced breakpoint lies within an Alu, a transposable element known to contribute to genomic arrangements (Rudiger et al., 1995). In ERG intron-3, the breakpoint resides in a hot spot clustered with genomic breakpoints previously identified in prostate cancer patients (Fig. S15B) (Weier et al., 2013). There is no obvious sequence homology between TMPRSS2 and ERG at the genomic breakpoint except for a three nucleotide 'CTG' microhomology (Fig 3E and S16), suggesting that this gene 
fusion may be mediated by non-homologous break repair mechanisms (Lieber, 2010; Zhang et al., 2009).

To test whether antisense input RNA can cause TMPRSS2-ERG fusion in non-malignant cells prior to cancerous transformation, we performed experiments using immortalized normal prostate epithelium cells (PNT1A), that express very low levels of androgen receptors (CollBastus et al., 2015). As shown in the lower panel of Fig. 3F, prolonged expression of antisense-5 for 12 days induced fusion transcripts (Sanger sequencing confirmation in Fig. S17). This induction was not due to prolonged exposure to DHT because continuous treatment of $0.9 \mu \mathrm{M}$ DHT alone for up to 2 months resulted in no detectable fusion transcripts in PNT1A cells (Fig. 3F, lane 8). Thus, our results indicate that the induction of TMPRSS2-ERG fusion by antisense input RNA can occur in normal prostate epithelial cells prior to malignant transformation and is not restricted to the pathological cellular context of malignant cells.

To test whether an input RNA can specify a pair of genes to undergo fusion other than TMPRSS2-ERG in a sequence-specific manner, we designed a series of input RNAs to induce TMPRSS2-ETV1, an inter-chromosomal fusion gene found in approximately $1 \%$ of prostate cancers (Rubin et al., 2011; Tomlins et al., 2005). Eight antisense RNAs (Fig. S18) were designed to target different chosen regions in the introns where three-way junctions potentially can be forged between the genomic DNA and input RNAs (Fig. S18 and S19). Again, because these input RNAs target introns and contain no exon sequence, it rules out the possibility that induced endogenous fusion transcripts composed of exons arise from the sequence of input RNAs or the plasmids. As shown in Fig. 4A, targeting stem TMPRSS2-ETV1-A, which has the highest genomic DNA stem stability $\left(\mathrm{Tm}=72^{\circ} \mathrm{C}\right)$ among this group, led to clear induction of the TMPRSS2-ETV1 fusion transcript (Fig. 4A, lane 1). Sanger sequencing validated that the induced transcript contains TMPRSS2 exon-1 joined with ETV1 exon-3 (uc003ssw.4) by annotated splice sites (Fig. S20). Similar to earlier observations, targeting with sense versions of input RNAs (Fig. 4B, lane 1 vs. 2), or using antisense input RNAs designed to form three-way junctions with lower genomic DNA stem stabilities (Fig. 4A, lanes 2 to 8; Fig. S18), resulted in no detectable induction. Furthermore, the input RNA designed to target TMPRSS2 and ETV1 induced TMPRSS2-ETV1 fusion but not TMPRSS2-ERG fusion (Fig. 4C, lane 2). Conversely, antisense-5 targeting TMPRSS2 and ERG induced TMPRSS2-ERG fusion but not TMPRSS2-ETV1 fusion (Fig. 4C, lane 1), indicating that fusion formation is specified by the sequence of input RNA and not secondary effects such as global genomic stability.

To verify that TMPRSS2-ETV1 as a second example of induced fusion that is indeed the consequence of genomic translocation, we propagated and enriched the induced LNCaP population for 47 days after the initial transfection of input RNA and DHT treatment (experimental procedures same as described for TMPRSS2-ERG enrichment in Fig. S14). The transiently expressed antisense input RNA had been degraded and was absent by day 47 (Fig. 
4D, lane 1 vs. 2). The induced TMPRSS2-ETV1 fusion transcript, however, was continuously expressed beyond day 47 (Fig. 4D, lane 2). Once again this observation indicated that the sustained expression of an induced fusion does not require the continuous presence of input RNA. Moreover, genomic PCR assays identified three distinct genomic breakpoints between TMPRSS2 and ETV1 gene (labeled as $\mathrm{x}, \mathrm{y}, \mathrm{z}$ in Fig. 4E) that were present only in the enriched LNCaP population but absent in untransfected LNCaP cells (Fig. 4E, lane 3 vs. 6, lane 9 vs. 12). Similar to earlier observations, no obvious sequence homology between TMPRSS2 and ETV1 was observed at the genomic breakpoints except for a few nt of microhomology (Fig. 4F, and Fig. S22 to S24), indicating that the gene fusion is mediated by non-homologous break repair mechanisms (Lieber, 2010; Zhang et al., 2009).

Unlike TMPRSS2 and ERG that are located near each other on the same chromosome, TMPRSS2 and ETV1 are located on different chromosomes. Thus, gene fusion as a result of chromosomal translocation could be confirmed unequivocally by evidence of chromosomal co-localization of the latter pair. Using probes specific to TMPRSS2 and ETV1, we performed fluorescence in situ hybridization (FISH) followed by deconvolution microscopic imaging of 3301 cells from the enriched LNCaP cell population and 620 cells from the control untransfected LNCaP population. Analyses of constructed 3D images showed that approximately $0.9 \%$ of the enriched population (30 out of 3301 cells) were positive for co-localization of TMPRSS2 and ETV1 gene in the cellular nucleus (Fig. 4G, examples of constructed 3D images are shown as movies in S25). In contrast, none of the cells from the untransfected population showed co-localized FISH signals as determined by the same 3D image criteria. These results, together with the genomic breakpoints identified by genomic PCR at single base resolution (Fig. 4E \& 4F), provide strong evidence that the induced expression of the TMPRSS2-ETV1 fusion transcript represents the consequence of gene fusion caused by chromosomal translocation.

One important observation emerging from our study is that all sense input RNAs failed to induce gene fusion. In particular, of the ten antisense input RNAs that were demonstrated to induce fusion (Fig. 1A, 1B, 2E, 4A), all of their corresponding sense counterparts failed to induce fusion (Fig. 1A, 1C, 2G, 4B). This specificity was oberserved despite the fact that sense input RNAs in theory can anneal to the same genomic sites targeted by their antisense counterparts and form similarly stable DNA/RNA hybrids. To test whether the disparity between antisense and sense is due to transcriptional activity of parental genes, we expressed the input RNAs by U6 (a pol-III promoter) for one day, followed by $\alpha$-amanitin-mediated inhibition of pol-II transcription for various time periods to shut down parental gene transcription. $\alpha$-amanitin was then removed to resume cellular transcription and the induction by sense vs. antisense input RNA were compared. As shown in Fig. 5 upper panel, the corresponding sense input RNAs that previously failed to induce fusion began to induce TMPRSS2-ERG after 12 hours of $\alpha$-amanitin treatment (lane 9 and 10), and TMPRSS2-ETV1 fusion (lane 20) after 24 hours of $\alpha$-amanitin treatment. This latent induction is not a property of general cellular toxicity of $\alpha$-amanitin because the toxicity caused 
by the same treatment actually reduced the induction by antisense input RNAs (lane 1 vs. 5 for TMPRSS2-ERG, lane 11 vs. 15 for TMPRSS2-ETV1). Furthermore, the input RNAs designed to target TMPRSS2 and ERG, regardless of their sense or antisense nature, induced TMPRSS2-ERG fusion but not that of TMPRSS2-ETV1 (lane 1 to 10). Conversely, input RNAs targeting TMPRSS2 and ETV1, regardless of their directional orientation, induced TMPRSS2-ETV1 fusion but not TMPRSS2-ERG fusion (lane 11 to 20). Additional control experiments using a parental plasmid vector lacking the input RNA sequences, DHT treatment without plasmid transfection, and PCR reactions without cDNA, all induced no endogenous fusion transcript under the same $\alpha$ amanitin treatment (Fig. 5, lower panel). The specificity exhibited by these experiments argues against general toxicity effects. The results suggest that the antisense versus sense disparity is largely due to transcriptional conflict.

With the plausibility of RNA-mediated gene fusion established, we then sought evidence that specific endogenous cellular RNAs can act as the 'initiator' to induce TMPRSS2-ERG fusion, which is found in $\sim 50 \%$ of prostate cancers. To identify candidate cellular initiator RNAs, we analyzed an available mRNA-seq database consisting of prostate tumors and matched benign tissues (Kannan et al., 2011). However, we found no evidence of perfect endogenous antisense chimeric RNAs in which the TMPRSS2 sequence was joined to any ERG sequence by discernable $5^{\prime}$ and $3^{\prime}$ splice sites in the antisense orientation. This suggests that if endogenous initiator RNAs do exist, they might arise from unrelated genomic sources that coincidentally resemble an imperfect chimeric RNA antisense to both TMPRSS2 and ERG. In a detailed study to be presented elsewhere (manuscript in preparation), we have performed thermodynamic calculations of RNA/DNA hybrids to identify cellular RNAs with partial sequence complementarity to the TMPRSS2 and ERG genes. We identified that AZI1 mRNA (also known as CEP131) (Aoto et al., 1997; Aoto et al., 1995) could form high affinity RNA/DNA hybrids with TMPRSS2 and ERG genomic sequences. As shown in Fig. 6A, overexpressing full-length AZI1 mRNA (3619 nt, uc002jzn.1) induced the TMPRSS2-ERG fusion transcript in LNCaP cells. The induction was observed at a physiologically relevant concentration (40 nM) of DHT (Fig. 6A, lane 4). Furthermore, expression of exon16-17 of AZI1, a short 220 nt segment containing an imperfect sequence antisense to TMPRSS2 and ERG, was sufficient to induce TMPRSS2-ERG fusion (Fig. 6B, lane 2). This result suggests that the induction of gene fusion is mediated by an RNA sequence that resides in exon16-17 and requires no AZI1 protein. Consistent with previous observations that sense input RNAs are ineffective for the fusion process, the expression of exon16-17 in the antiparallel orientation also failed to induce TMPRSS2-ERG fusion (Fig. 6B, lane 3). 


\section{$\underline{\text { Discussion }}$}

In summary, this report provides the first evidence that expression of a chimeric RNA can drive the formation of gene fusions in mammalian cells. Hence, we propose that "the cart before the horse" hypothesis concerning fusion gene causation is mechanistically plausible. Our data support a model (shown in Fig. 7) where the initiator RNA with chimeric sequence invades chromosomal DNA to stabilize a transient RNA/DNA duplex using DNA sequences located in two distant genes. Resolution of such an RNA/DNA duplex by DNA repair mechanisms might yield the final gene fusion through recombination in regions prone to DNA breaks (Fig. 7A). Such events were rare in the initial population of transfected cells ( 1 in $10^{3}$ or $10^{4}$ cells occurred within 3 days). However, the necessary machinery is clearly present in normal prostate epithelial cells prior to malignant transformation. If the resulting gene fusion (such as TMPRSS2-ERG) provides a growth advantage, a single affected cell among billions of cells in a normal prostate tissue may proliferate abnormally and eventually contribute to cancer formation. Identifying such initiator RNAs might provide novel insights into early disease mechanisms, as well as the discovery of new preventive and therapeutic strategies to combat cancer.

Contrary to the previous "cart before the horse" model (Rowley and Blumenthal, 2008; Zaphiropoulos, 2011), our results do not support the postulation that a sense fusion mRNA derived from trans-splicing between two pre-mRNAs effectively directs gene fusion. Expressing sense input RNAs mirroring the trans-spliced mRNA failed to induce fusion in LNCaP cells (Fig. 1A and S2). Of ten antisense RNAs that were demonstrated to be capable of inducing fusion (Fig. 1A, 1B, 2E, 4A, 6B), all of their corresponding sense RNAs failed to induce fusion (Fig. 1A, 1C, 2G, 4B, 6B). This occurred even though the sense RNAs could, in theory, anneal to the same genomic sites targeted by their antisense counterparts and form similar DNA/RNA hybrids when paired with the antisense strand of genomic DNA. As demonstrated in Fig. 5, this antisense versus sense disparity can be explained by transcriptional conflict (Fig. 7B and 7C). Because the TMPRSS2 promoter is highly active in LNCaP cells, sense chimeric RNAs forming DNA/RNA hybrids with antisense strands of genomic DNA (the template strand used for transcription) would be frequently "bumped" off and unable to stabilize the structures required for initiating genomic arrangements. The mechanistic basis for the antisense versus sense RNA, and whether these phenomena can be generalized, remain to be investigated.

Our results also do not support the hypotheses that antisense input RNAs, acting as a docking station, mediate trans-splicing by base-pairing with both endogenous sense parental pre-mRNAs, or by bringing the parental genes in close proximity thus facilitating trans-splicing of parental pre-mRNAs transcribed from two genomic loci. Both mechanisms would require the continuous presence of antisense input RNAs to sustain the expression of induced fusion transcripts. Yet we showed that the induced fusion expression has a permanent nature and requires no continuous presence of input RNAs (Fig. 3B and Fig. 4D). Furthermore, in the case of TMPRSS2-ERG there is no detectable ERG parental RNA as raw material (Fig. 3A) to account for the trans-splicing 
models. Moreover, sense input RNAs, which are not complimentary to the sense parental premRNAs thus cannot act as their docking station, are able to induce the fusion transcripts after a brief period of transcriptional inhibition, again arguing against the docking model. On the contrary, the genomic breakpoints identified by genomic PCR and chromosomal co-localization provided by FISH, strongly support that the induced expression of fusion transcript is largely the consequence of gene fusion resulting from chromosomal translocation. While prior works have shown that infrequent TMPRSS2-ERG fusions can by induced through genotoxic stress such as gamma radiation in the presence of DHT that increases double-stranded DNA breaks (Lin et al., 2009; Mani et al., 2009), such mechanisms of general genotoxicity fail to account for the specificity of gene fusion partners found in cancer. This report is the first to demonstrate RNAmediated gene fusion in mammalian cells, and provides an RNA-driven mechanism that can account for the 'specificity' of gene fusion partners that were selected to undergo gene fusion in early disease stages. The results may represent a pathological example in a broad spectrum of potential RNA-mediated genome rearrangements and could have fundamental implications in the biology of mammalian genome stability, as well as gene editing technology via mechanisms native to mammalian cells.

\section{Author contributions}

SKG and LL conducted the experiments, SKG and LY designed the experiments, SKG and LY conceived the project and wrote the manuscript.

\section{Acknowledgments}

We thank Richard Kelley, Michael Ittmann, Richard Sifers and Tom Cooper for critical suggestions, Christine Shiang for cloning AZI1 ex16-17, Olga Dakhova, Jianghua Wang, and Michael Ittmann for providing the cell lines. Sachin Kumar Gupta has been supported by CPRIT training grant RP160283. Laising Yen has been supported by Duncan Cancer Center Pilot Grant, CPRIT HIHRRA RP160795, and NIH R01EB013584. We would also like to thank Radhika Dandekar, Fabio Stossi, and Michael Mancini for assisting with microscopy, with the support by the Integrated Microscopy Core at Baylor College of Medicine with funding from the NIH (DK56338, and CA125123), CPRIT (RP150578) the Dan L. Duncan Comprehensive Cancer Center, and the John S. Dunn Gulf Coast Consortium for Chemical Genomics. 


\section{$\underline{\text { References }}$}

Aoto, H., Miyake, Y., Nakamura, M., and Tajima, S. (1997). Genomic organization of the mouse AZ1 gene that encodes the protein localized to preacrosomes of spermatids. Genomics 40, 138141.

Aoto, H., Tsuchida, J., Nishina, Y., Nishimune, Y., Asano, A., and Tajima, S. (1995). Isolation of a novel cDNA that encodes a protein localized to the pre-acrosome region of spermatids. Eur $\mathrm{J}$ Biochem 234, 8-15.

Bastus, N.C., Boyd, L.K., Mao, X., Stankiewicz, E., Kudahetti, S.C., Oliver, R.T., Berney, D.M., and Lu, Y.J. (2010). Androgen-induced TMPRSS2:ERG fusion in nonmalignant prostate epithelial cells. Cancer Res 70, 9544-9548.

Boyce, M.J., Baisley, K.J., Clark, E.V., and Warrington, S.J. (2004). Are published normal ranges of serum testosterone too high? Results of a cross-sectional survey of serum testosterone and luteinizing hormone in healthy men. in BJU Int, pp. 881-885.

Coll-Bastus, N., Mao, X., Young, B.D., Sheer, D., and Lu, Y.J. (2015). DNA replicationdependent induction of gene proximity by androgen. Hum Mol Genet 24, 963-971.

Crick, F. (1970). Central dogma of molecular biology. Nature 227, 561-563.

Fang, W., and Landweber, L.F. (2012). RNA-mediated genome rearrangement: hypotheses and evidence. Bioessays 35, 84-87.

Horoszewicz, J.S., Leong, S.S., Chu, T.M., Wajsman, Z.L., Friedman, M., Papsidero, L., Kim, U., Chai, L.S., Kakati, S., Arya, S.K., et al. (1980). The LNCaP cell line--a new model for studies on human prostatic carcinoma. Prog Clin Biol Res 37, 115-132.

Janz, S., Potter, M., and Rabkin, C.S. (2003). Lymphoma- and leukemia-associated chromosomal translocations in healthy individuals. Genes Chromosomes Cancer 36, 211-223.

Kannan, K., Wang, L., Wang, J., Ittmann, M.M., Li, W., and Yen, L. (2011). Recurrent chimeric RNAs enriched in human prostate cancer identified by deep sequencing. Proc Natl Acad Sci U S A $108,9172-9177$.

Kimsey, I., and Al-Hashimi, H.M. (2014). Increasing occurrences and functional roles for high energy purine-pyrimidine base-pairs in nucleic acids. Curr Opin Struct Biol 24, 72-80.

Langabeer, S.E., Walker, H., Rogers, J.R., Burnett, A.K., Wheatley, K., Swirsky, D., Goldstone, A.H., and Linch, D.C. (1997). Incidence of AML1/ETO fusion transcripts in patients entered into the MRC AML trials. MRC Adult Leukaemia Working Party. Br J Haematol 99, 925-928.

Li, H., Wang, J., Mor, G., and Sklar, J. (2008). A neoplastic gene fusion mimics trans-splicing of RNAs in normal human cells. Science 321, 1357-1361.

Lieber, M.R. (2010). The mechanism of double-strand DNA break repair by the nonhomologous DNA end-joining pathway. Annu Rev Biochem 79, 181-211.

Lin, C., Yang, L., Tanasa, B., Hutt, K., Ju, B.G., Ohgi, K., Zhang, J., Rose, D.W., Fu, X.D., Glass, C.K., et al. (2009). Nuclear receptor-induced chromosomal proximity and DNA breaks underlie specific translocations in cancer. Cell 139, 1069-1083. 
Mani, R.S., Tomlins, S.A., Callahan, K., Ghosh, A., Nyati, M.K., Varambally, S., Palanisamy, N., and Chinnaiyan, A.M. (2009). Induced chromosomal proximity and gene fusions in prostate cancer. Science 326, 1230.

Mitelman, F., Johansson, B., and Mertens, F. (2007). The impact of translocations and gene fusions on cancer causation. Nat Rev Cancer 7, 233-245.

Nowacki, M., Vijayan, V., Zhou, Y., Schotanus, K., Doak, T.G., and Landweber, L.F. (2008). RNA-mediated epigenetic programming of a genome-rearrangement pathway. Nature 451, 153158.

Perner, S., Demichelis, F., Beroukhim, R., Schmidt, F.H., Mosquera, J.M., Setlur, S., Tchinda, J., Tomlins, S.A., Hofer, M.D., Pienta, K.G., et al. (2006). TMPRSS2:ERG fusion-associated deletions provide insight into the heterogeneity of prostate cancer. Cancer Res 66, 8337-8341.

Rowley, J.D., and Blumenthal, T. (2008). Medicine. The cart before the horse. Science 321, 1302-1304.

Rubin, M.A., Maher, C.A., and Chinnaiyan, A.M. (2011). Common gene rearrangements in prostate cancer. J Clin Oncol 29, 3659-3668.

Rudiger, N.S., Gregersen, N., and Kielland-Brandt, M.C. (1995). One short well conserved region of Alu-sequences is involved in human gene rearrangements and has homology with prokaryotic chi. Nucleic Acids Res 23, 256-260.

Tomlins, S.A., Rhodes, D.R., Perner, S., Dhanasekaran, S.M., Mehra, R., Sun, X.W., Varambally, S., Cao, X., Tchinda, J., Kuefer, R., et al. (2005). Recurrent fusion of TMPRSS2 and ETS transcription factor genes in prostate cancer. Science 310, 644-648.

Watson, J.D., and Crick, F.H. (1953). Genetical implications of the structure of deoxyribonucleic acid. Nature 171, 964-967.

Weier, C., Haffner, M.C., Mosbruger, T., Esopi, D.M., Hicks, J., Zheng, Q., Fedor, H., Isaacs, W.B., De Marzo, A.M., Nelson, W.G., et al. (2013). Nucleotide resolution analysis of TMPRSS2 and ERG rearrangements in prostate cancer. J Pathol 230, 174-183.

Zaphiropoulos, P.G. (2011). Trans-splicing in Higher Eukaryotes: Implications for Cancer Development? Front Genet 2, 92.

Zhang, F., Carvalho, C.M., and Lupski, J.R. (2009). Complex human chromosomal and genomic rearrangements. Trends Genet 25, 298-307. 


\section{Figure 1}

A.

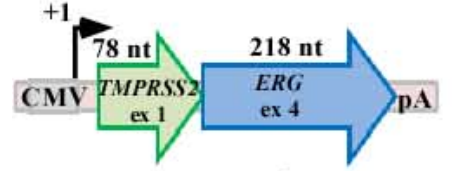

sense-1

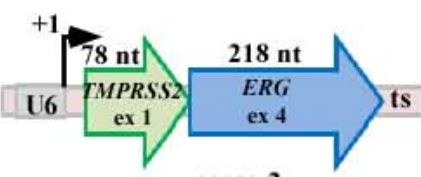

sense-2
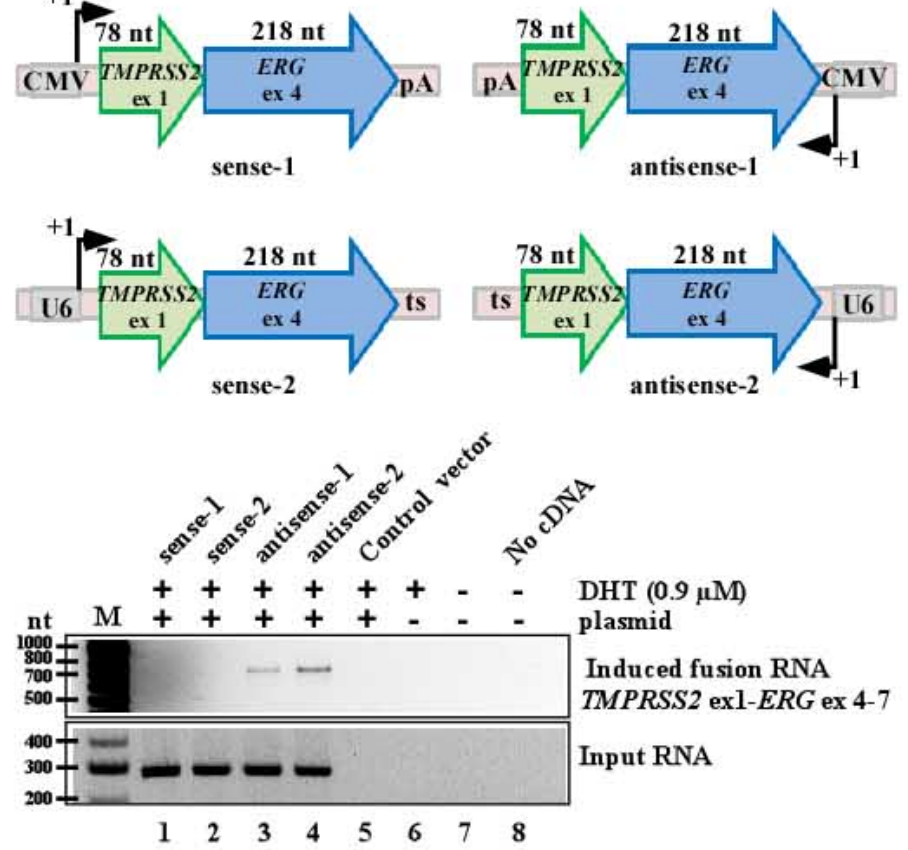

C.

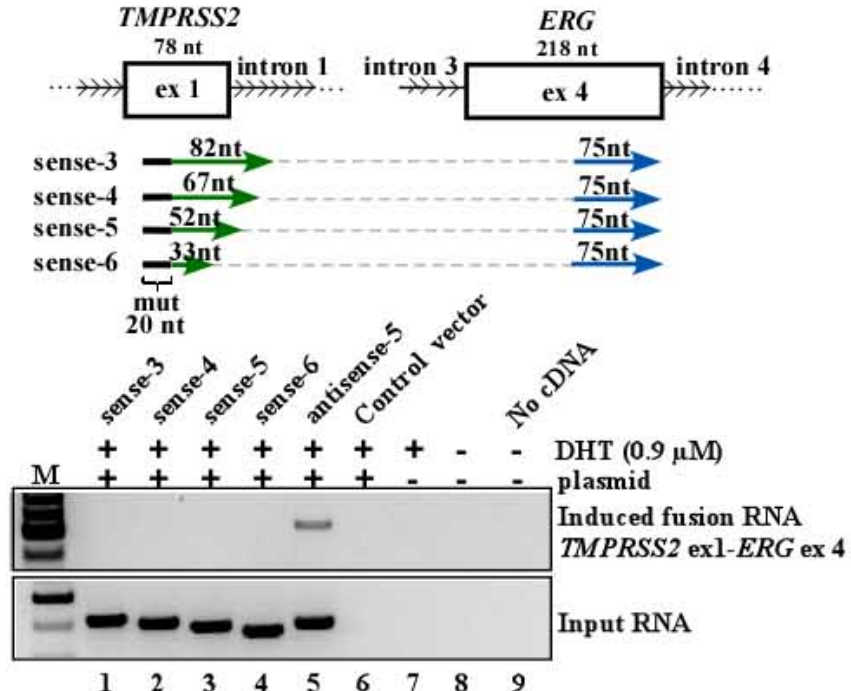

E.

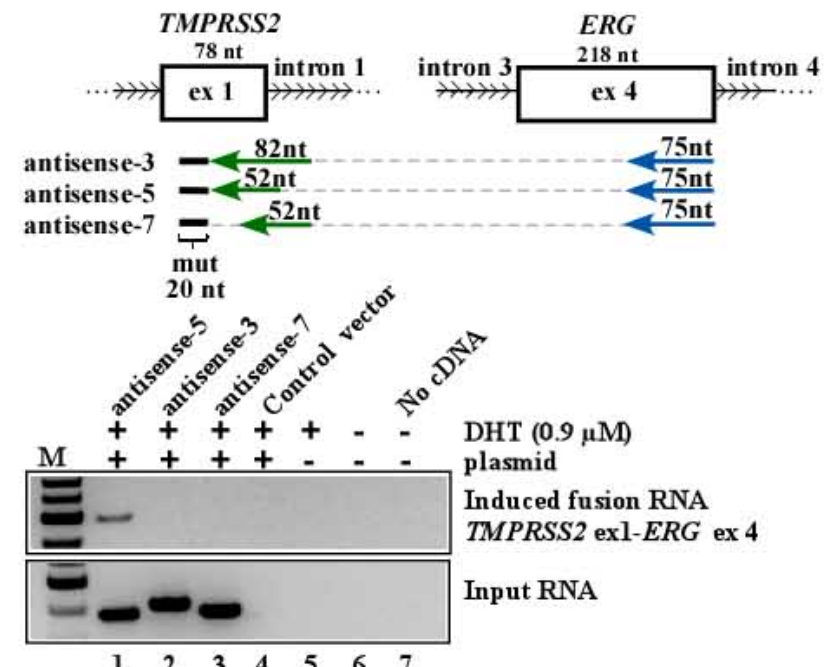

B.

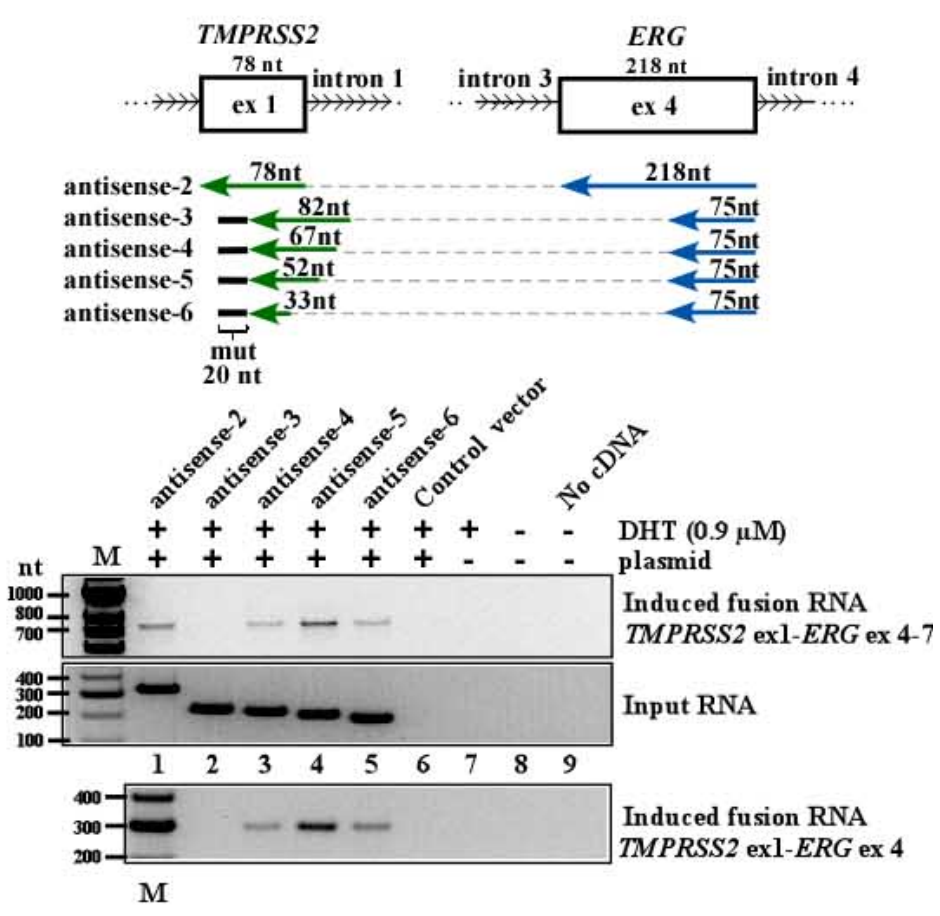

D.

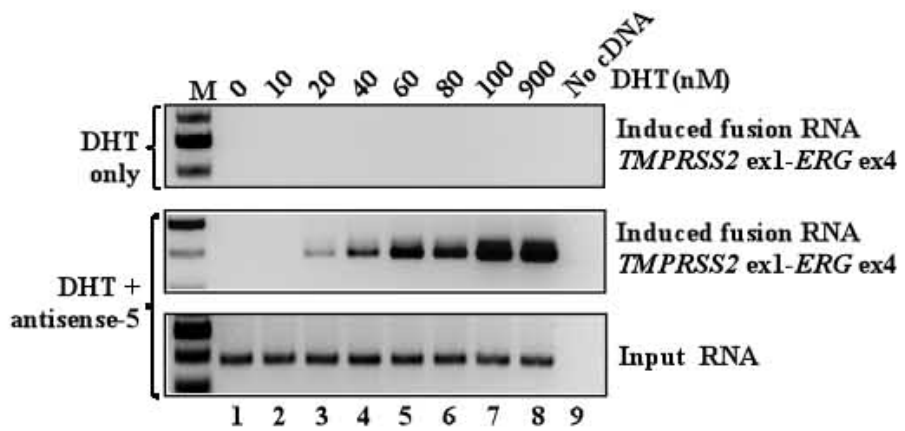


Fig 1. Exogenously expressed input chimeric RNAs induce the expression of endogenous fusion transcripts.

A. Upper: Schematics of the designed input RNAs containing complete TMPRSS2 exon-1 (78 nt, uc002yzj.3) and ERG exon-4 (218 nt, uc021wjd.1), expressed in the sense or antisense orientation from the CMV or U6 promoters. pA: poly-A signal. ts: transcriptional stop “TTTTTT” for U6 promoter. Lower: RT-PCR detection of induced fusion transcript (top gel) and input RNA (bottom gel). Parental plasmid vector containing mCherry sequence, DHT treatment without plasmid transfection, and PCR reaction without cDNA served as the controls (land 5, 6, 8). B. Length and positional effect of antisense input RNAs. Upper: Antisense input RNAs with 75 nt (blue) targeting ERG exon-4 and varying lengths (82, 67, 52, and 33 nt, green) targeting TMPRSS2. Dashed line links ERG and TMPRSS2 sequence within the input RNA and contains no sequence. A 20-nt mutation (black line) was introduced to the input RNAs to discern expressed input RNAs from the induced fusion transcript (see Fig. S1B). Lower: RT-PCR detection of induced fusion transcript (top gel), input RNA (middle gel), or detection of induced fusion transcript using a different primer pair (bottom gel). C. Corresponding sense input RNAs all failed to induce the fusion transcript. D. Induction by antisense-5 occurred at physiologically relevant DHT concentrations as low as $20 \mathrm{nM}$. Three-rounds of nested PCR were performed to reveal the lowest amount of DHT required. E. Antisense-5 led to clear induction while antisense3 and 7 did not, indicating that it is not the length of input RNAs but targeted regions that is critical. 
bioRxiv preprint doi: https://doi.org/10.1101/151241; this version posted June 16,2017 . The copyright holder for this preprint (which was not certified by peer review) is the author/funder, who has granted bioRxiv a license to display the preprint in perpetuity. It is made available under

\section{Figure 2}

A.

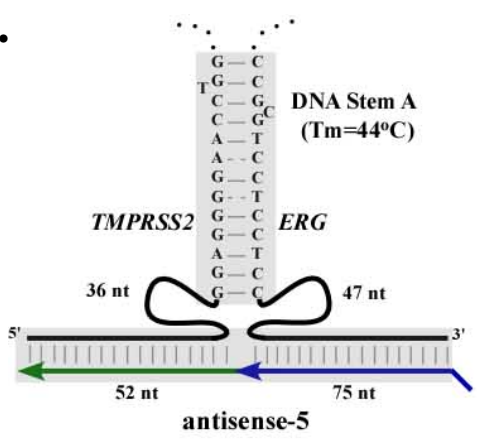

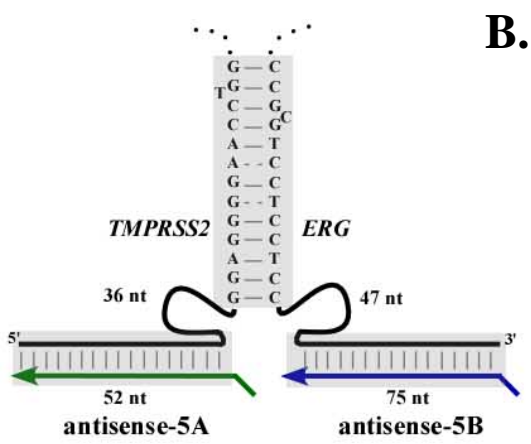

antisense-5B

B.

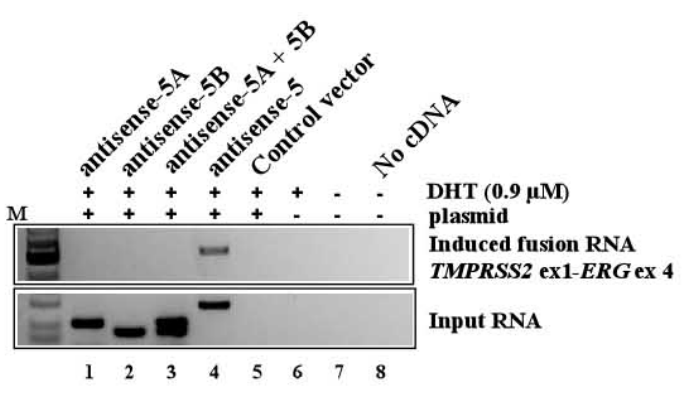

C.

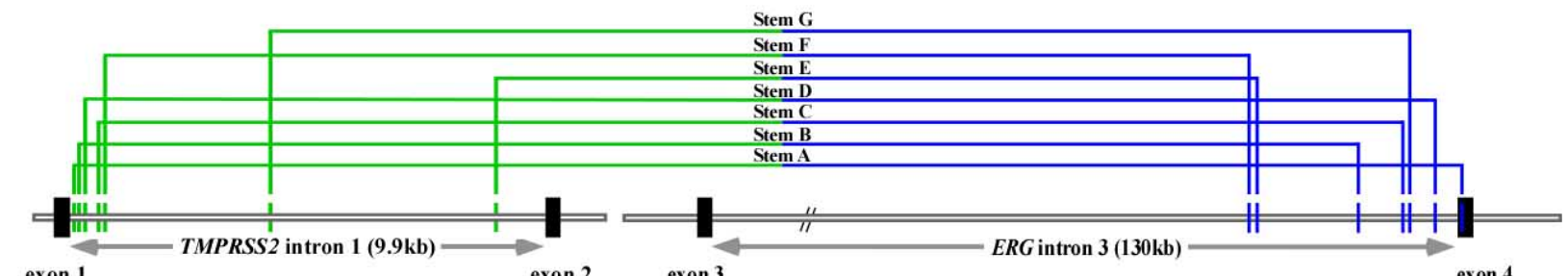
exon 1

exon 2

D.

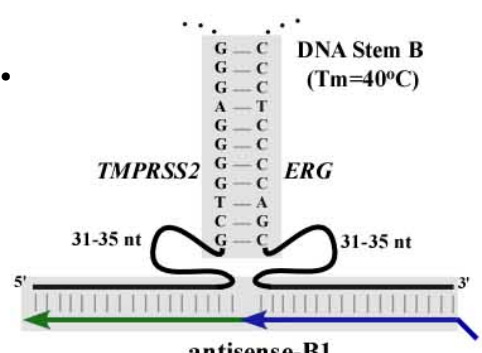

antisense-B1

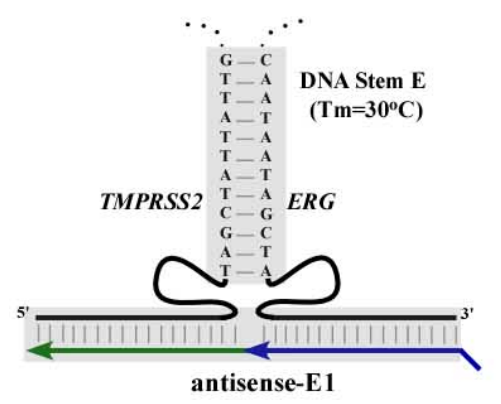

E.

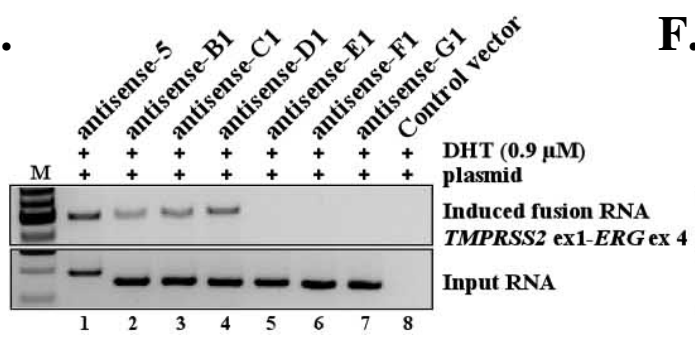

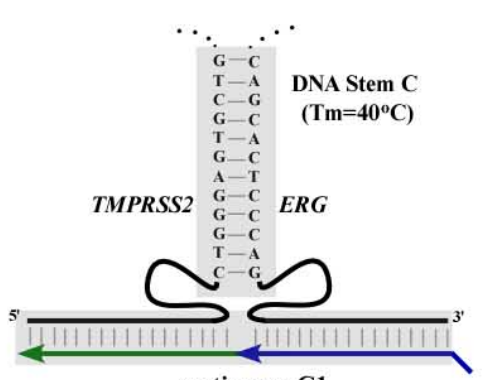

antisense-C1

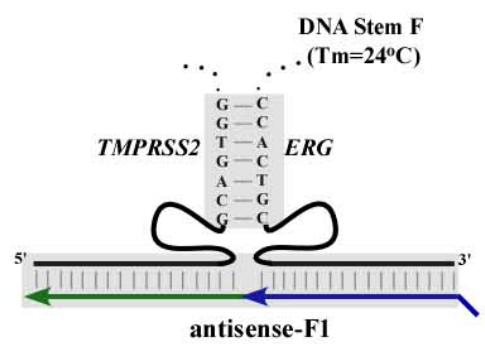

F.

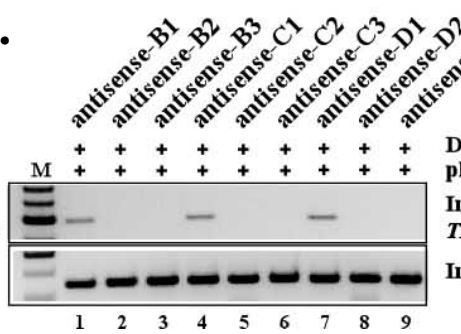

exon 4

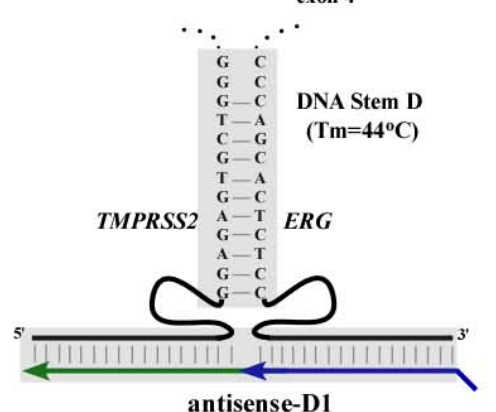

antisense-D1

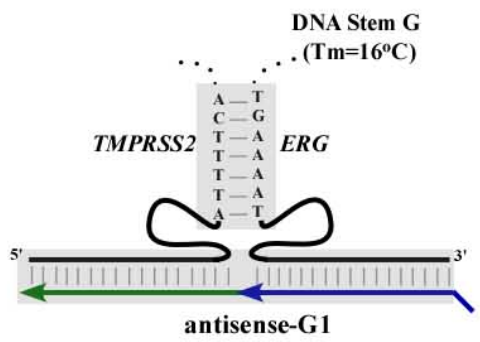

G.

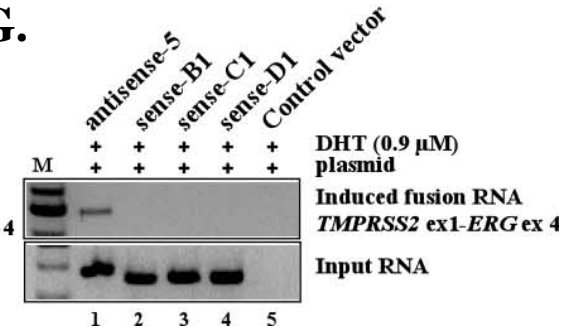


Fig 2. Formation of a three-way junction may facilitate fusion induction.

A. Left: Schematics of three-way junction that could be formed between genomic DNA (black) and antisense-5 input RNA (green/blue). The sense genomic strands of both TMPRSS2 and ERG genes are on the minus strand of chromosome 21, separated by $3 \mathrm{Mb}$. Short lines in shaded regions represent base-pairings. Imperfect stem A includes a high energy $\mathrm{G} \cdot \mathrm{T}$ and $\mathrm{A} \cdot \mathrm{C}$ wobble pair known to have Watson-Crick-like geometry in a DNA double helix (Kimsey and AlHashimi, 2014; Watson and Crick, 1953). A spacer region of $36 \mathrm{nt}$ and $47 \mathrm{nt}$ separate stem A from the regions targeted by antisense-5 input RNA. Right: Expressing antisense-5 as two separate halves that severed the link between TMPRSS2 (52 nt) and ERG (75 nt) sequence within the input RNA. B. RT-PCR assays of fusion transcripts showed that the severed input RNAs resulted in the loss of induction. C. Locations of putative stems A to F identified by BLAST analyses. Genomic coordinates are listed in Fig. S9. D. The putative three-way junction formed between the indicated genomic DNA stem B to G (black) and designed antisense input RNA (green/blue). E. Targeting genomic DNA stem B, C, and D that exhibit higher DNA stem stability $\left(\mathrm{Tm}=40^{\circ} \mathrm{C}, 40^{\circ} \mathrm{C}\right.$, and $44^{\circ} \mathrm{C}$, respectively) by the corresponding antisense input RNAs induced fusion transcripts (lanes 2 to 4). In contrast, targeting less stable stem E, F, and G (Tm= $30^{\circ} \mathrm{C}, 24^{\circ} \mathrm{C}$, and $16^{\circ} \mathrm{C}$, respectively) failed to induce fusion transcripts (lanes 5 to 7 ). $\mathbf{F}$. Antisense input RNAs designed to invade each of the respective genomic DNA stem B, C, and D resulted in the loss of induction. G. Corresponding sense input RNAs targeting stem B, C, and D failed to induce fusion transcripts (lanes 2 to 4 ). 
bioRxiv preprint doi: https://doi.org/10.1101/151241; this version posted June 16, 2017. The copyright holder for this preprint (which was not certified by peer review) is the author/funder, who has granted bioRxiv a license to display the preprint in perpetuity. It is made available under

\section{$\underline{\text { Figure } 3}$} aCC-BY 4.0 International license.

A.

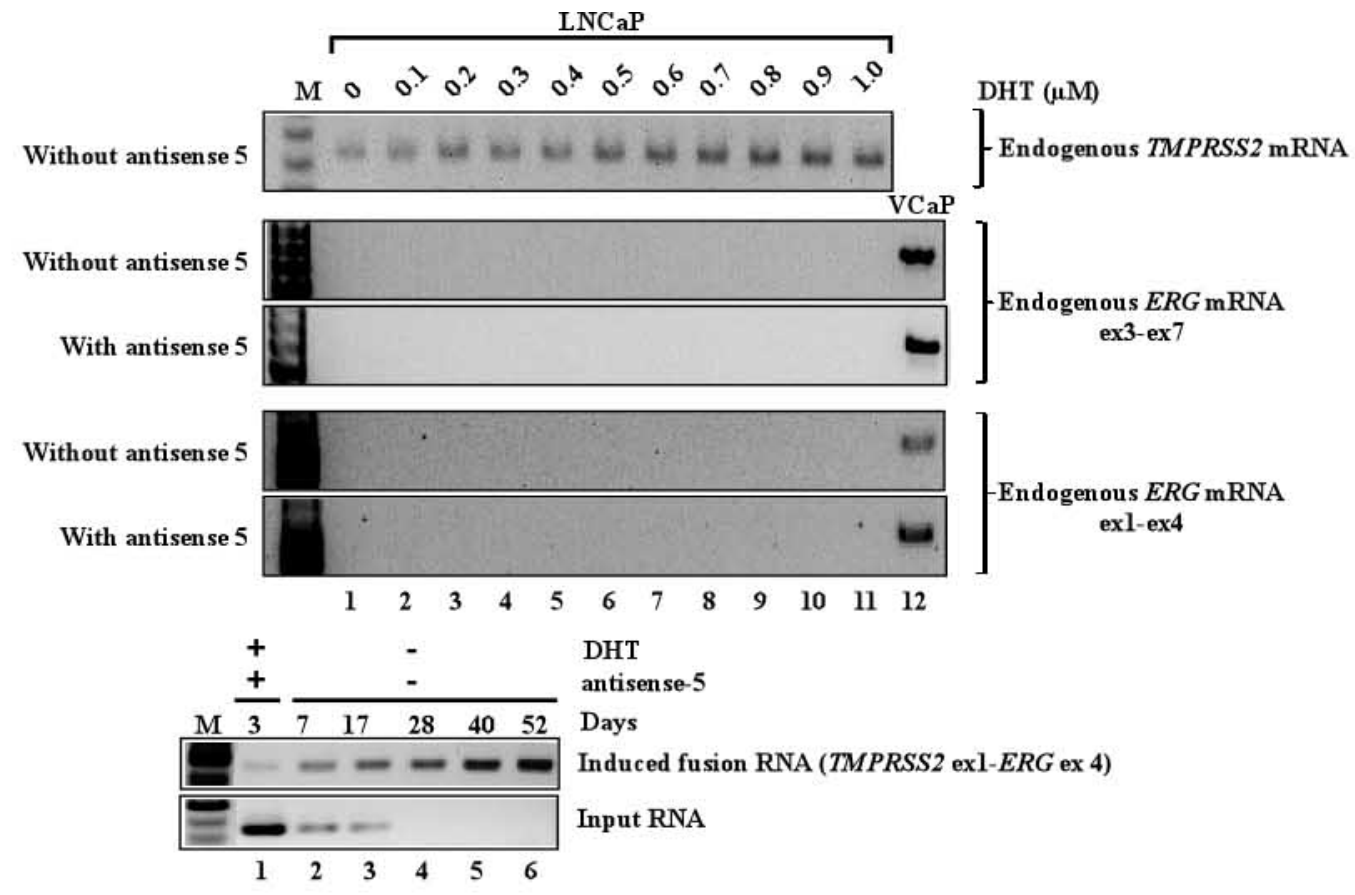

C.

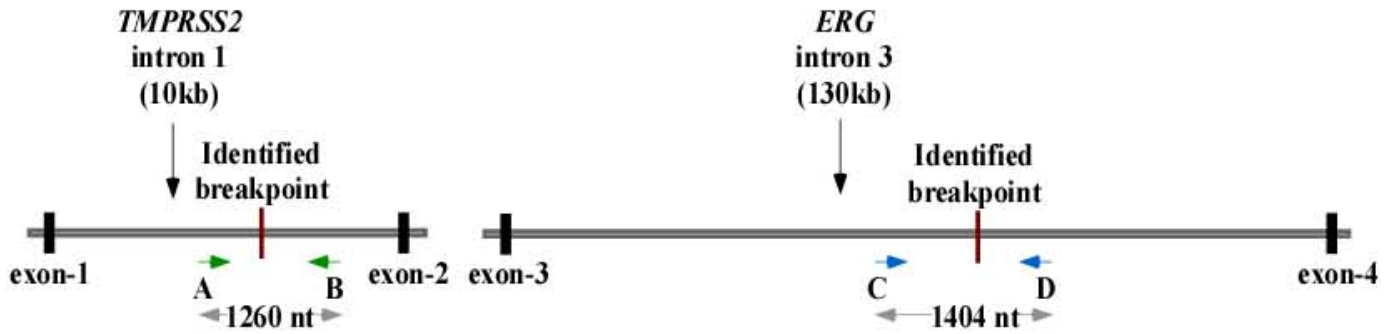

D.

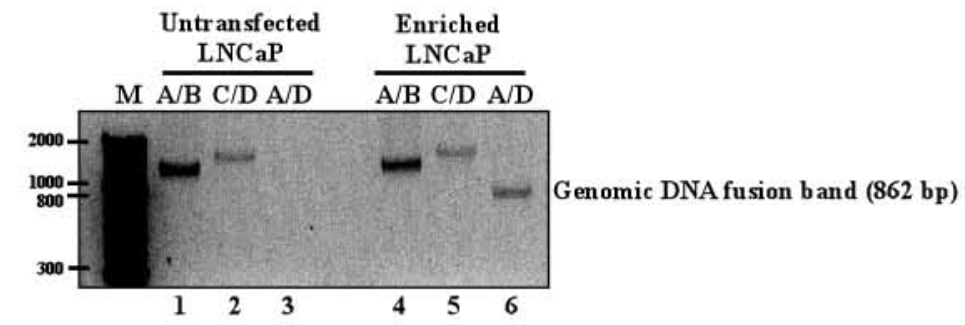

E.

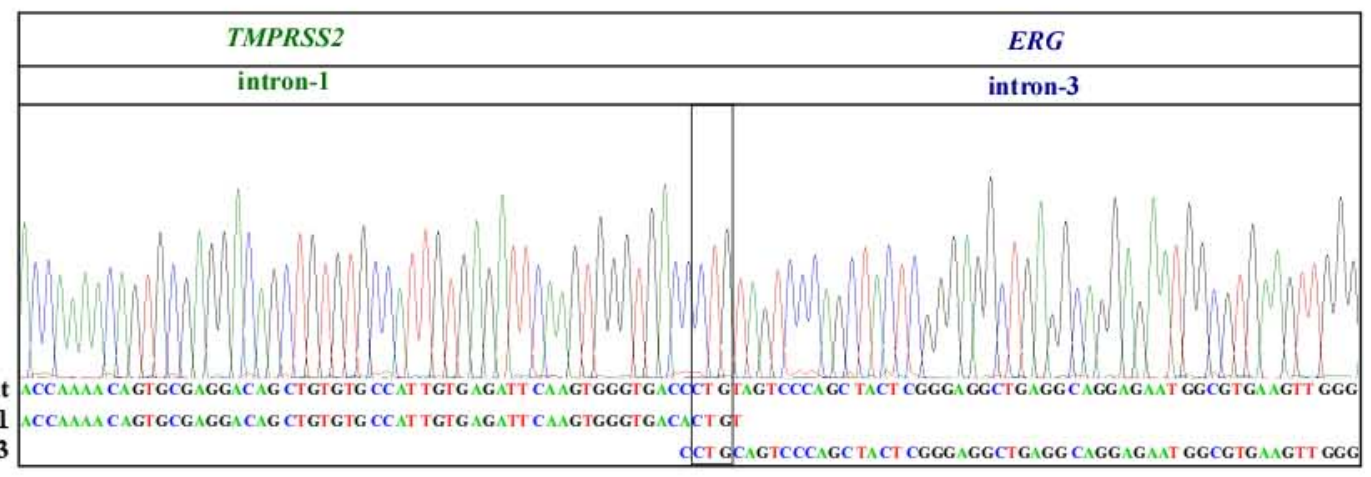

F.

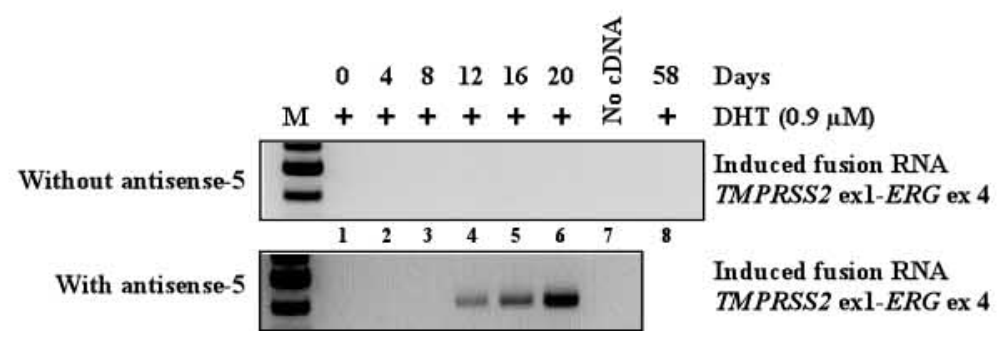




\section{Fig 3. Induced TMPRSS2-ERG fusion is the result of genomic arrangements}

A. Upper: RT-PCR shows that LNCaP cells express TMPRSS2 mRNA, and that the expression is upregulated by DHT. Primers used are specific to TMPRSS2 exon-2 and exon-4. Middle and lower: ERG mRNA however was not detected in LNCaP cells under a wide range of DHT in the presence or absence of antisense-5 (lanes 1 to 11). Assays were performed using two independent primer pairs, one specific to exon-3 and -7 (middle panel), the other to exon-1 and 4 (lower panel), both capable of detecting ERG mRNA in VCaP cells (lane 12). These primer pairs were chosen because they selectively amplified endogenous ERG mRNA but not the induced TMPRSS2-ERG fusion transcript which has ERG exon-3 to exon-12. B. RT-PCR shows the transient nature of input RNA (lower panel) and the persistent nature of the induced fusion transcript (upper panel) in the enriched LNCaP population up to 52 days post initial treatment (see Fig. S14 for enrichment procedure). C. Schematics of identified genomic breakpoints and the primers used to amplify the breakpoints. The approximate location of genomic breakpoints were first determined using a primer set targeting TMPRSS2 intron-1 multiplexed with a primer set targeting ERG intron-3 (see Fig. S15A). The region near the breakpoint was then further examined using primers A, B, C, and D. D. The un-rearranged wildtype TMPRSS2 and ERG alleles were revealed by primer pair A/B ( 1404 bp) and C/D ( 1260 bp) respectively (lane 1, 2, $4,5)$. The genomic fusion band of 862 bp amplified by fusion-specific primer pair A/D was present only in the enriched LNCaP population (lane 6) and absent in untransfected LNCaP cells (lane 3). E. Sanger sequencing of the fusion band showed a 500bp segment of TMPRSS2 intron1 fused to 362 bp of ERG intron-3. The genomic breakpoint contains a 'CTG' microhomology (boxed). The full-length Sanger sequence is shown in S16. F. Prolonged expression of antisense5 for 12 days induced the TMPRSS2-ERG fusion transcript in PNT1A cells as detected by threeround nested PCR. The results indicate that the induction of TMPRSS2-ERG fusion by antisense input RNA can occur in normal prostate epithelial cells prior to malignant transformation. 


\section{Figure 4} aCC-BY 4.0 International license.
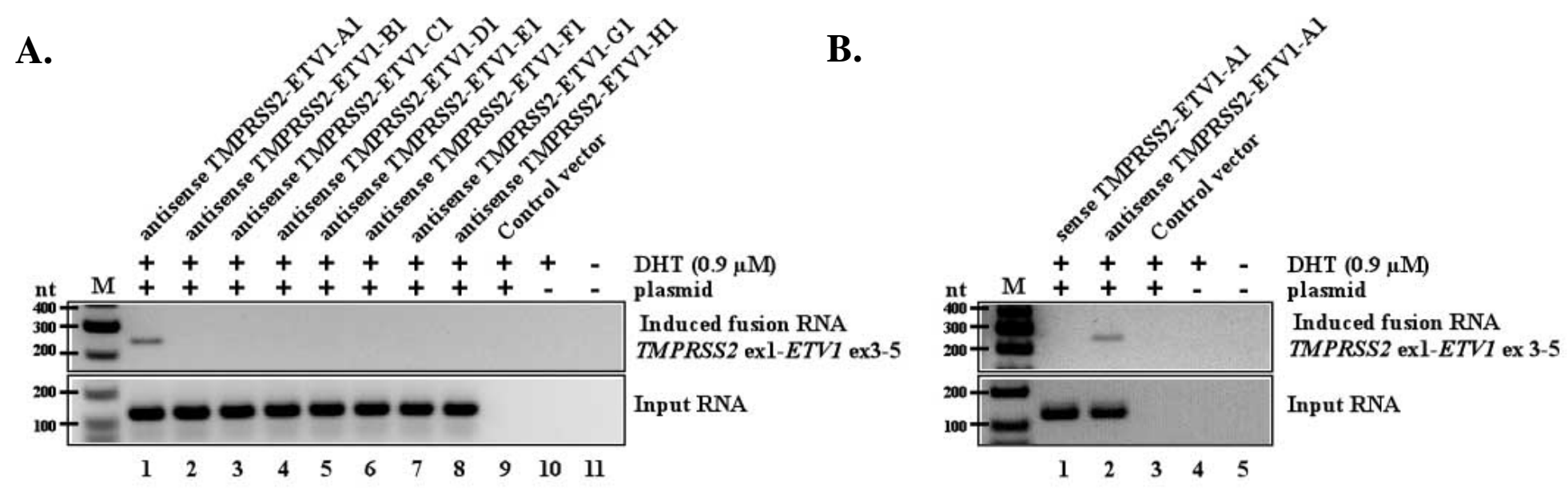

C.

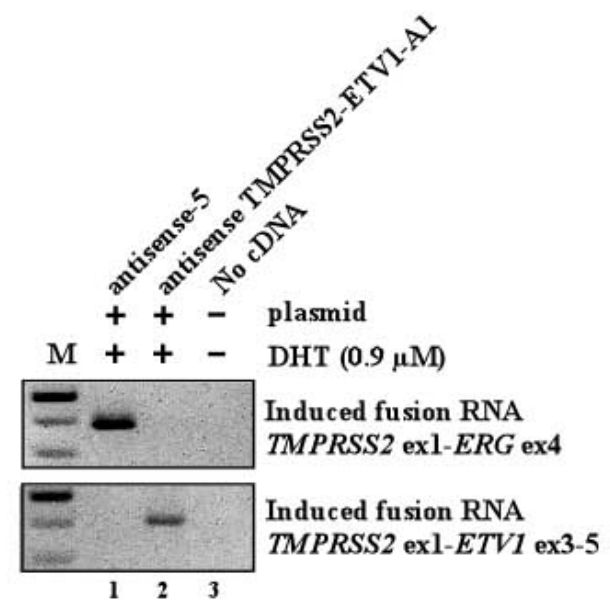

D.

\section{TMPRSS2}

E.

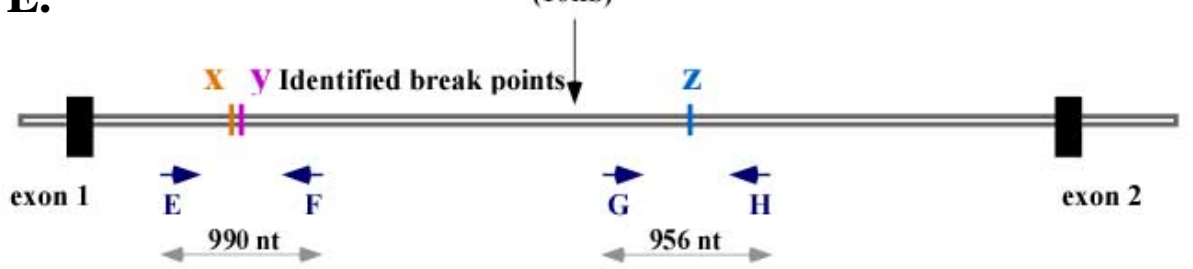

ETVI

intron 2

(0.8kb)
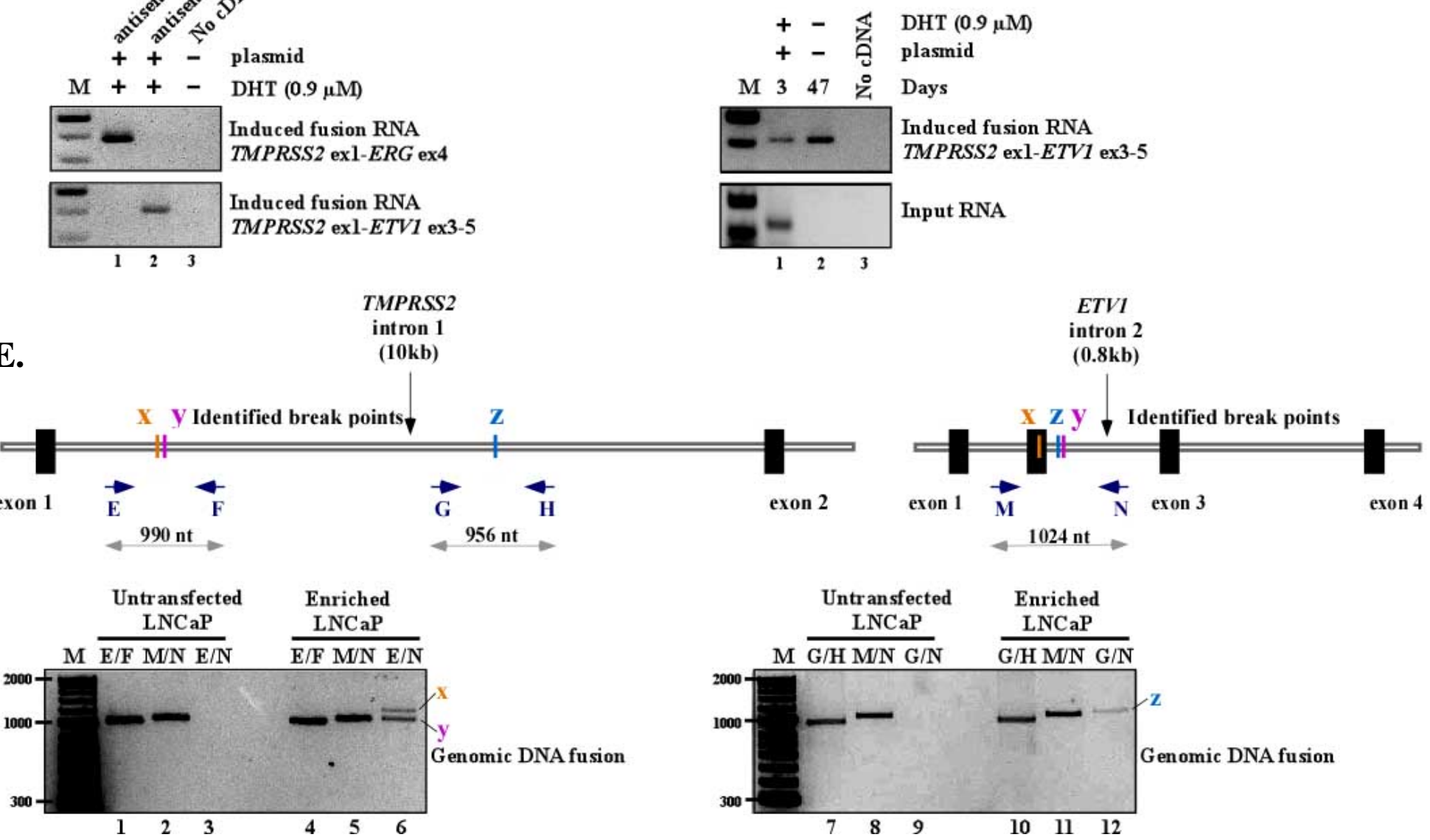
bioRxiv preprint doi: https://doi.org/10.1101/151241; this version posted June 16, 2017. The copyright holder for this preprint (which was not certified by peer review) is the author/funder, who has granted bioRxiv a license to display the preprint in perpetuity. It is made available under

\section{Figure 4 continued} aCC-BY 4.0 International license.

F.
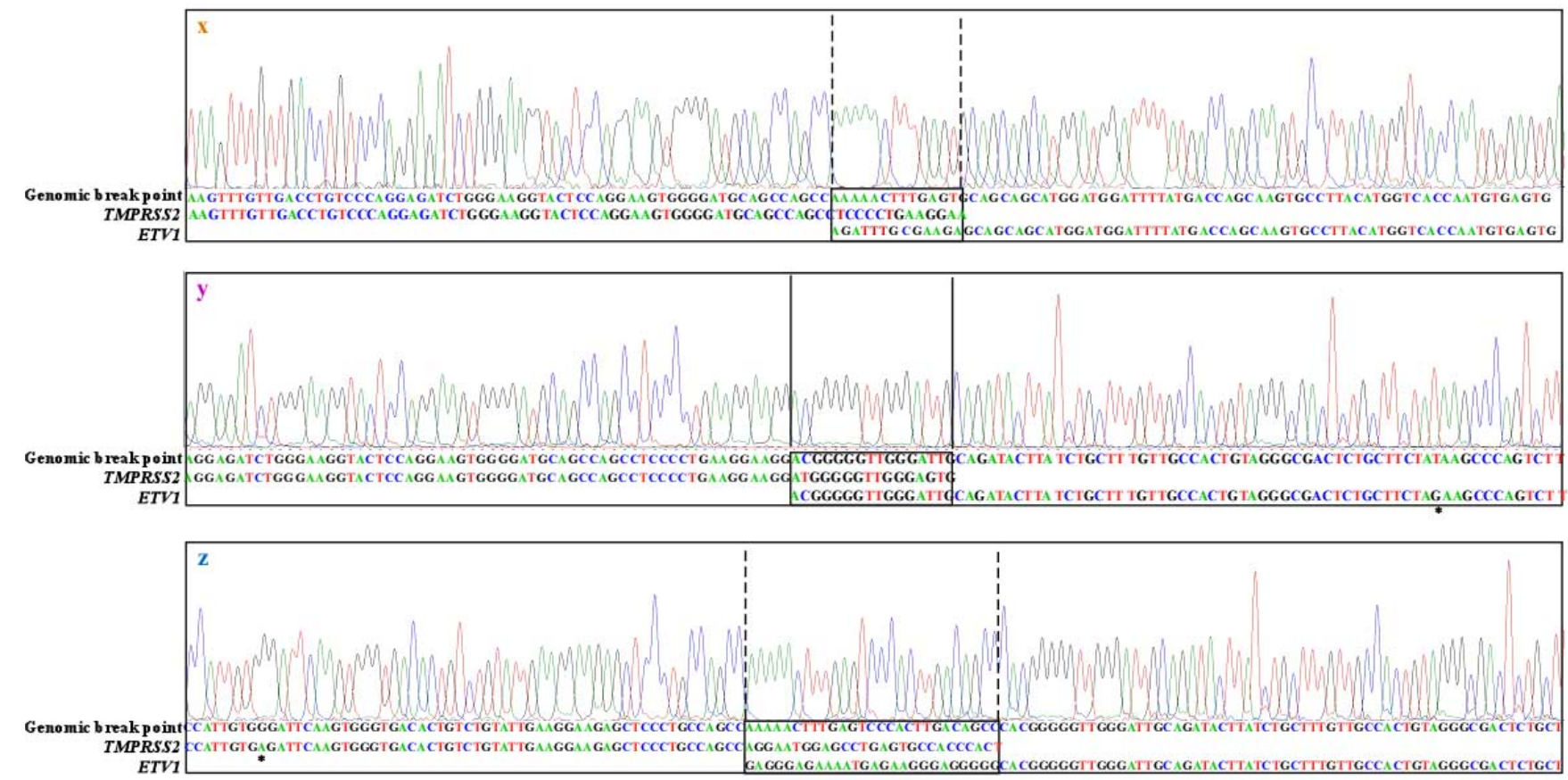

G.
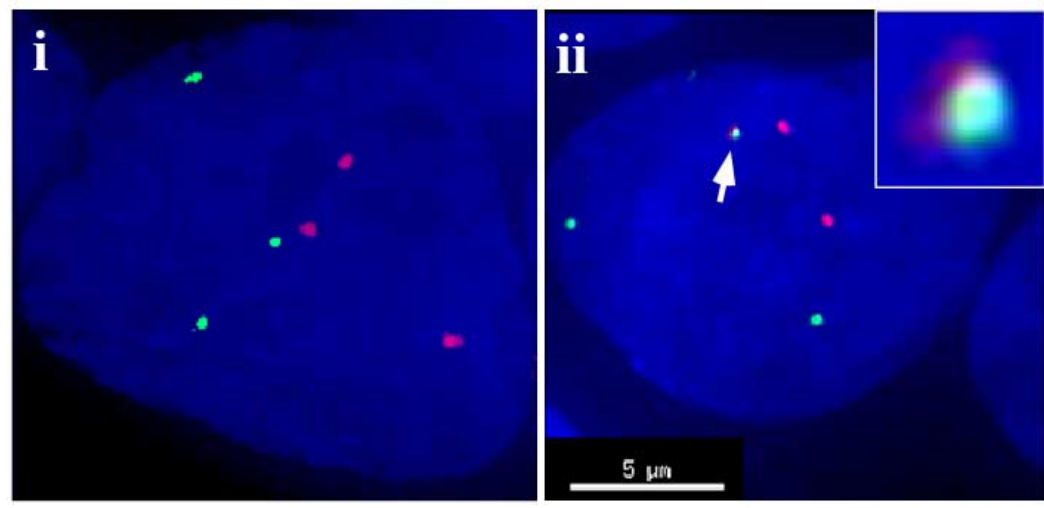

\begin{tabular}{|c|c|c|}
\hline & $\begin{array}{c}\text { Untransfeted } \\
\text { LNCaP cells }\end{array}$ & $\begin{array}{c}\text { Enriched } \\
\text { LNCaP population }\end{array}$ \\
\hline $\begin{array}{c}\text { Total No. of } \\
\text { imaged cells }\end{array}$ & 620 & 3301 \\
\hline $\begin{array}{c}\text { No. of cells } \\
\text { positive for } \\
\text { fusion genes }\end{array}$ & 0 & 30 \\
\hline$\%$ & 0 & 0.91 \\
\hline
\end{tabular}


Fig 4. RNA-mediated inter-chromosomal gene fusion between TMPRSS2 and ETV1.

A. RT-PCR shows that only the antisense RNA designed to target stem TMPRSS2-ETV1-A, which has the highest stem stability $\left(\mathrm{Tm}=72^{\circ} \mathrm{C}\right)$, led to induced fusion transcript (lane 1). Antisense RNAs targeting locations with lower genomic DNA stem stabilities (lanes 2 to 8), resulted in no detectable induction. B. The corresponding sense input RNA targeting the same TMPRSS2-ETV1-A stem failed to induce fusion transcript (lane 1 vs. 2). C. Gene fusion is specified by the sequence of input RNA used. The input RNA targeting TMPRSS2 and ETV1 induced TMPRSS2-ETV1 fusion but not TMPRSS2-ERG fusion (lane 2). Conversely, antisense-5 targeting TMPRSS2 and ERG induced TMPRSS2-ERG fusion but not TMPRSS2-ETV1 fusion (lane 1). D. RT-PCR shows the transient nature of input RNA which was present at day 3 but not day 47 post initial treatment (lower panel, lane 1 vs. 2), and the persistent nature of the induced fusion transcript (upper panel) up to 47 days post initial treatment in the enriched LNCaP population. E. Schematics of three identified genomic breakpoints marked as $\mathrm{x}, \mathrm{y}$, and z. The approximate location of genomic breakpoints were first determined using a primer set targeting TMPRSS2 intron-1 multiplexed with a primer set targeting ETV1 intron-2 (see Fig. S21). The unrearranged wildtype TMPRSS2 allele was then revealed by primer pair E/F (990 bp; lane 1 and 4) and G/H (956 bp; lane 7 and 10), and the un-rearranged wildtype ETV1 allele by primer pair M/N (1024 bp; lane 2, 5, 8, 11). The genomic fusion band x (1150 bp) and y (1044 bp) amplified by fusion-specific primer pair E/N, and z (1043 bp) amplified by primer pair G/N, were present only in the induced and enriched LNCaP population but absent in untransfected LNCaP cells (lane 6 vs. 3, and lane 6 vs. 12). F. Sanger sequencing of the $\mathrm{x}, \mathrm{y}$, and $\mathrm{z}$ fusion band identified the exact genomic breakpoints. Region of microhomology at the breakpoints are boxed by solid lines, and indels by dash lines. The full-length Sanger sequences are shown in Fig. S22 to S24. G. FISH combined with 3D image reconstruction confirmed the gene fusion between TMPRSS2 with ETV1 in the enriched population. FISH probes against TMPRSS2 gene (red) located on chromosome 21 and against ETV1 gene (green) located on chromosome 7 were used to reveal gene fusion based on the co-localized FISH signals in reconstructed 3D images. Examples of FISH signal in an untransfected cell (i) and a cell carrying induced TMPRSS2-ETV1 gene fusion (ii) are shown. Arrow points to the co-localized FISH signals indicative of TMPRSS2-ETV1 fusion, which is shown at a higher magnification in the inlet. The table on the right shows the population analysis based on the 3D construction of FISH images. Approximately $0.9 \%$ of the enriched population (30 out of 3301 cells) was positive for TMPRSS2-ETV1 fusion gene based on the co-localized FISH signals. In contrast, none of the cells from the untransfected population ( 0 out 620 cells) showed co-localized FISH signals. 
bioRxiv preprint doi: https://doi.org/10.1101/151241; this version posted June 16,2017 . The copyright holder for this preprint (which was not certified by peer review) is the author/funder, who has granted bioRxiv a license to display the preprint in perpetuity. It is made available under aCC-BY 4.0 International license.

\section{$\underline{\text { Figure } 5}$}

TMPRSS2-ERG $[$ 1st round

TMPRSS2-ETV1 $[3$ rd round
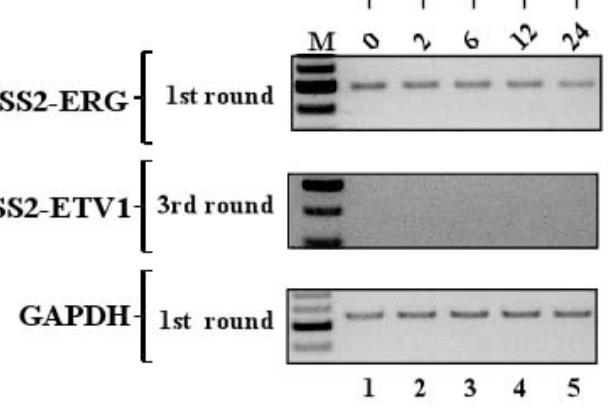
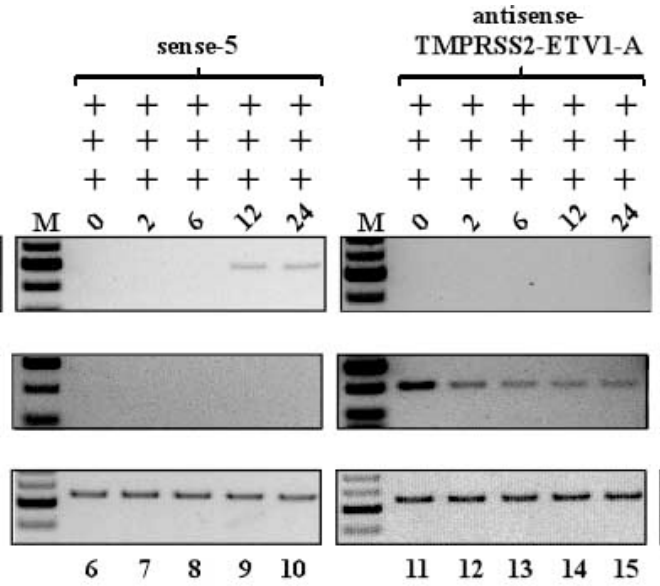
TMPRSS2-ETV1-A

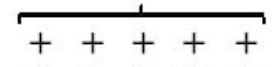

$+++++$

$+++++$
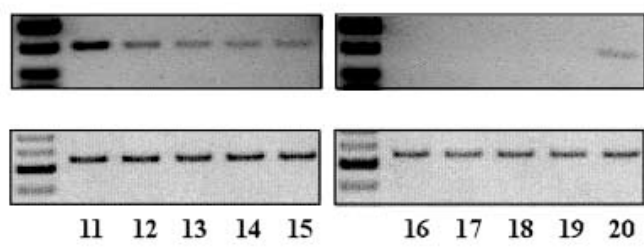
TMPRSS2-ETV1-A
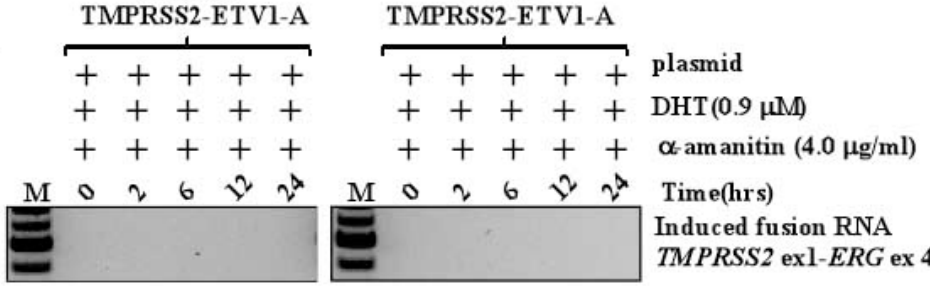

Time(hrs)

Induced fusion RNA

TMPRSS 2 exl-ERG ex 4

Induced fusion RNA

TMPRSS2 exl-ETV1 ex 3-5

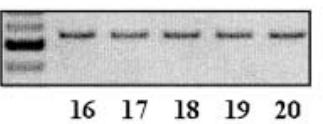

Parental plasmid control

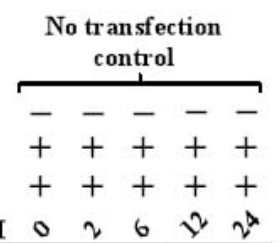

TMPRSS2-ERG $[$ lst round

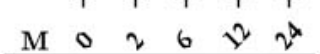

TMPRSS2-ETV1 $[$ 3rd round
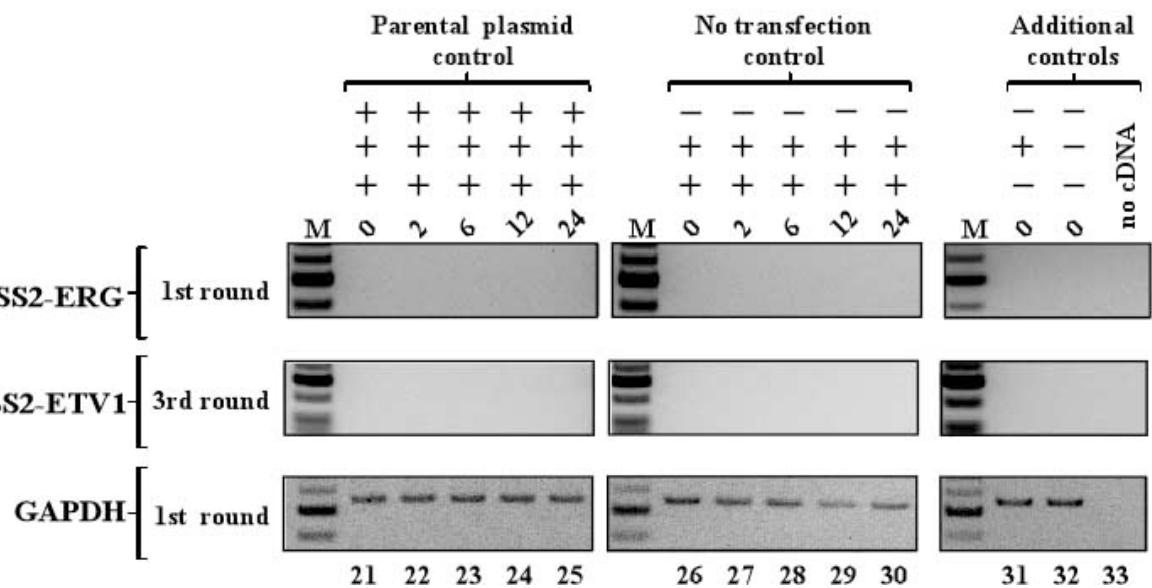

plasmid

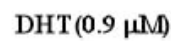

$\alpha$-amanitin $(4.0 \mu \mathrm{g} / \mathrm{ml})$

Time(hrs)

Induced fusion RNA

TMPRSS 2 exl-ERG ex 4

Induced fusion RNA

TMPRSS2 exl-ETV1 ex 3-5

$\begin{array}{lll}31 & 32 & 33\end{array}$ 
Fig 5. The disparity between antisense versus sense input RNA is due to transcriptional conflict.

The input RNAs were expressed by U6 (a pol-III promoter) for one day, followed by $\alpha$-amanitinmediated inhibition of pol-II transcription for various time periods $(0,2,6,12$ and 24 hrs) to shut down parental gene transcription. $\alpha$-amanitin was then removed to resume cellular transcription and the induction by sense vs. antisense input RNA were compared. The corresponding sense input RNAs that previously failed to induce fusion, began to induce TMPRSS2-ERG (lane 9, 10) after 12 hours of $\alpha$-amanitin treatment, and TMPRSS2-ETV1 fusion (lane 20) after 24 hours of $\alpha$ amanitin treatment, respectively. The results indicate that blocking the parental gene transcription by $\alpha$-amanitin for a longer period (12-24 hrs for TMPRSS2-ERG and 24 hrs for TMPRSS2-ETV1) allows sense input RNA to induce gene fusion. Input RNAs used for the experiment: Antisense-5 vs. sense-5 for inducing TMPRSS2-ERG (upper panel, left two columns); antisense vs. sense TMPRSS2-ETV1-A for inducing TMPRSS2-ETV1 (upper panel, right two columns). As controls, transfecting cells with a parental plasmid containing no input RNA sequences (lower panel, left column), cells without transfection (lower panel, center column), and DHT and $\alpha$-amanitin controls (lower panel, right column), also failed to induce fusion. In addition, GAPDH is used as internal control for the amount of RNA in each lane. 


\section{$\underline{\text { Figure } 6}$} aCC-BY 4.0 International license.

A.

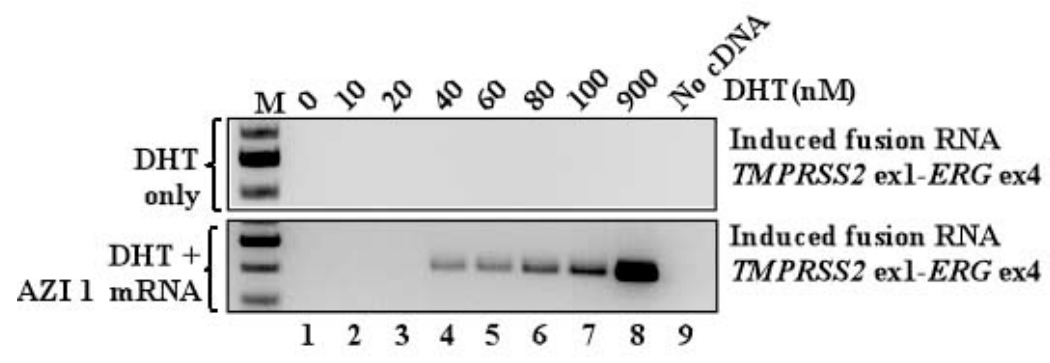

C.

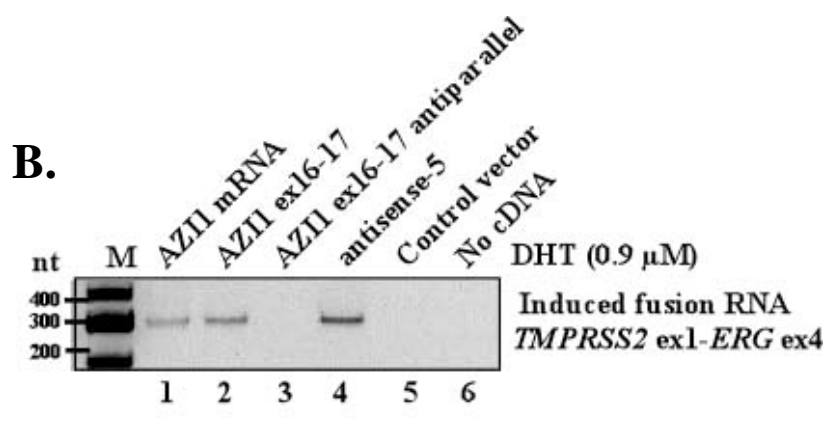

DHT ( $\mu \mathrm{M})$ 
Fig 6. Specific endogenous RNA acts as the 'initiator' RNA to mediate gene fusion.

A. Expression of full-length AZI1 mRNA for 3 days induced TMPRSS2-ERG fusion. The induction occurred at physiologically relevant concentrations of DHT as low as $40 \mathrm{nM}$ (lane 4). Three-rounds of nested PCR were performed to reveal the lowest amount of DHT that permits AZI1-mediated fusion induction. B. Expression of AZI1 exon 16-17 (lane 2), but not its antiparallel sequence (lane 3), led to TMPRSS2-ERG fusion. Inductions by full-length AZI1 (lane 1) and by antisense-5 (lane 4) were used as positive controls. C. Endogenous AZI1 RNA is expressed in LNCaP cells at low level and the expression is upregulated by DHT treatment. 


\section{Figure-7}

A.
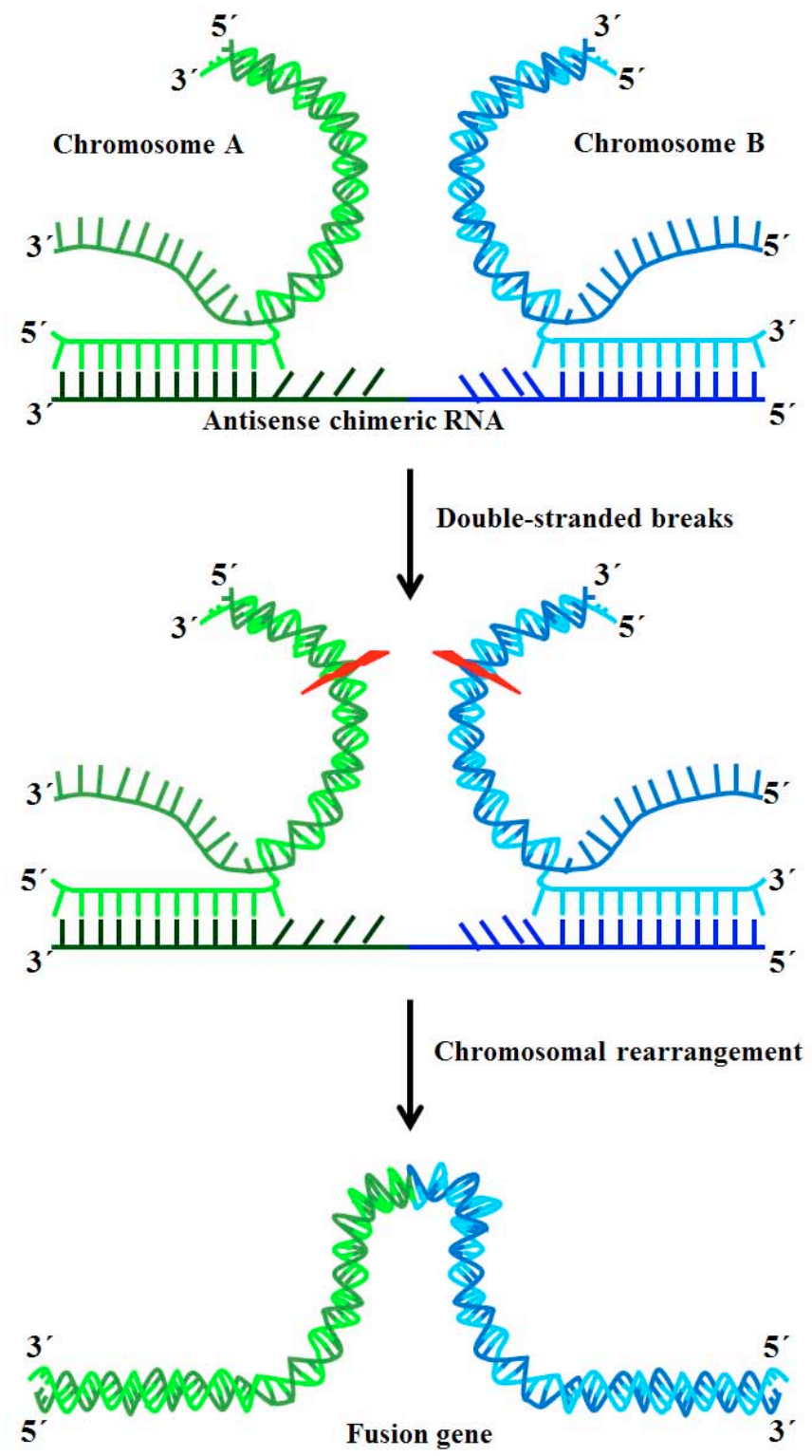

B.

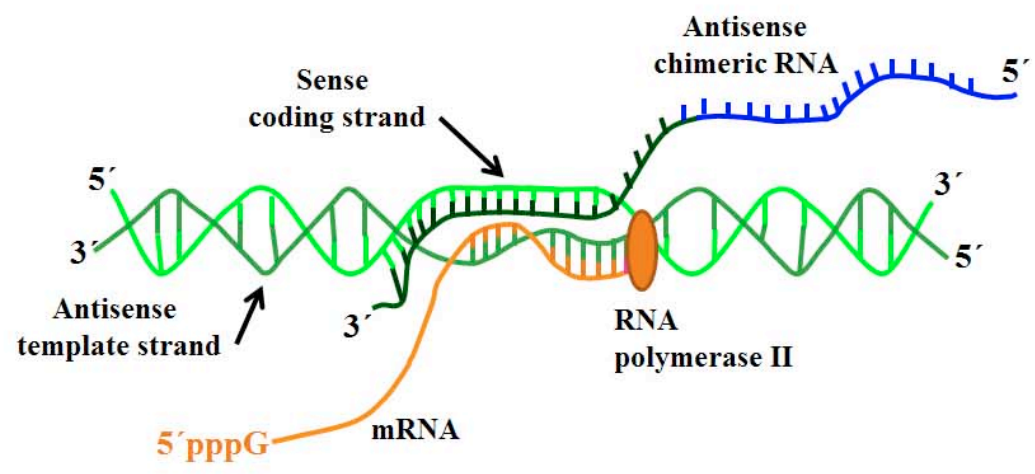

C.

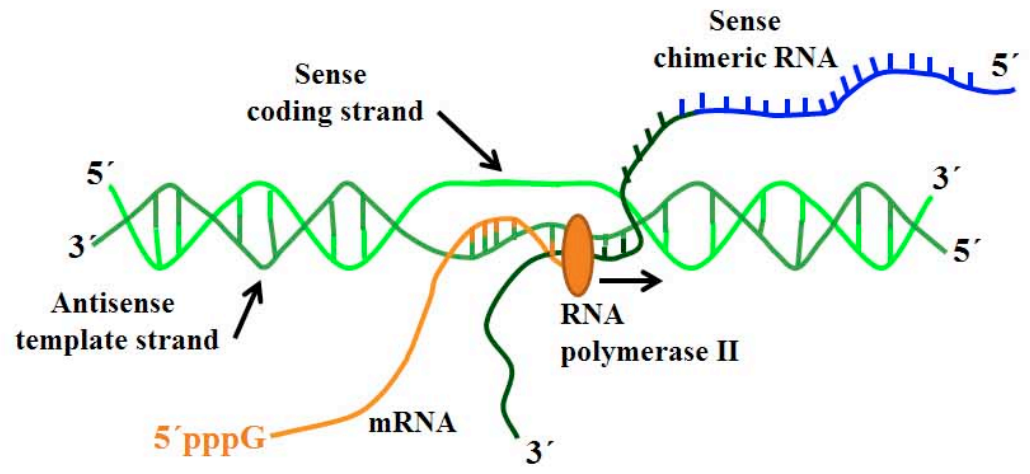


Fig 7. A model of RNA-mediated gene fusion in mammalian cells.

A. Our data support a model where the initiator RNA with chimeric sequence invades chromosomal DNA to stabilize a transient RNA/DNA duplex using DNA sequences located in two distant genes. Resolution of such an RNA/DNA duplex by DNA break/repair mechanisms might yield the final gene fusion through recombination in regions prone to DNA breaks. Such events however are infrequent ( 1 in $10^{3}$ or $10^{4}$ cells in the experimental cell population), and are dependent of the presence of hormone DHT in the case of LNCaP cells. B and C. The data also indicate that it is the antisense rather than sense chimeric RNAs that effectively drive gene fusion, and that this disparity can be explained by transcriptional conflict produced by the transcriptional activity of parental genes. The sense chimeric RNAs forming DNA/RNA hybrids with antisense strands of genomic DNA (the template strand used for transcription) are likely be frequently "bumped" off by RNA polymerase and unable to stabilize the structures required for initiating genomic arrangements. The proposed RNA-driven model may provide a mechanism that can 'specify' gene fusion partners in early disease stages, and could have fundamental implications in the biology of mammalian genome stability, as well as gene editing technology via mechanisms native to mammalian cells. 


\section{Supplementary Information}

\section{RNA-mediated Genomic Arrangements in Mammalian Cells}

Sachin Kumar Gupta, Liming Luo, and Laising Yen*

Department of Pathology \& Immunology, Department of Molecular and Cellular Biology, Dan

L. Duncan Cancer Center, Baylor College of Medicine, Houston, TX 77030, USA 
A.

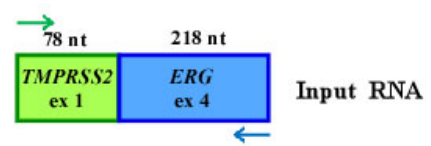

\begin{tabular}{|c|c|c|c|c|c|c|c|c|}
\hline $78 \mathrm{nt}$ & $218 \mathrm{nt}$ & $152 \mathrm{nt}$ & $204 \mathrm{nt}$ & $81 \mathrm{nt}$ & $72 \mathrm{nt}$ & $69 \mathrm{nt}$ & $388 \mathrm{nt}$ & \\
\hline $\begin{array}{c}\text { TMPRSS2 } 2 \\
\text { ex } 1\end{array}$ & $\begin{array}{c}E R G \\
\text { ex } 4\end{array}$ & $\begin{array}{c}E R G \\
\text { ex } 5\end{array}$ & $\begin{array}{l}E R G \\
\text { ex } 6\end{array}$ & $\begin{array}{c}E R G \\
\text { ex } 7\end{array}$ & $\begin{array}{c}E R G \\
\text { ex } 8\end{array}$ & $\begin{array}{r}E R G \\
\text { ex } 9\end{array}$ & $\begin{array}{l}E R G \\
\text { ex } 12\end{array}$ & Induced fusion RNA \\
\hline
\end{tabular}

B.

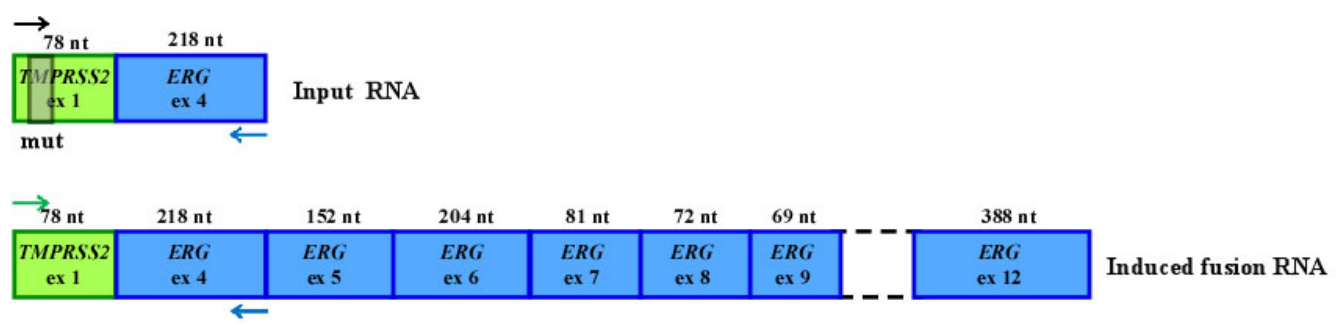

5' GAGTAGGCCGAGCTAAGCAGGAGGGGAGGCGAGGCGAGGGCG......... Wild type TMPRSS2 exon1

5' GAGTAGGCGCGAGCTTCCATCTCTCACTCCTGTCTGGCGGAGGGCG.......... Mutated TMPRSS2 exon1

Fig. S1. Primer design used to distinguish induced endogenous fusion RNAs from exogenous input RNAs.

A. The endogenous full-length TMPRSS2-ERG fusion RNA most commonly found in prostate cancer consisting of TMPRSS2 exon 1 (78 nt, uc002yzj.3) spliced to ERG exons 4-12 (1289 nt, uc021wjd.1). In Fig. 1A in the main text, the exogenous input RNAs expressed from plasmids consists of TMPRSS2 exon-1 (78 nt) fused to ERG exon-4 (218 nt). The RT-PCR results shown in Fig. $1 \mathrm{~A}$ and $1 \mathrm{~B}$ (middle panel) in the main text were performed using a forward primer on TMPRSS2 exon-1 paired with a reverse primer on ERG exon-7, thereby specifically amplifying the endogenous fusion RNA transcribed from the TMPRSS2-ERG fusion gene, but not from transfected plasmids or input RNAs because both lack ERG exon-7. B. Because RNA-induced fusion is a low frequency event, we developed a more efficient PCR method to detect induced endogenous fusion RNA using a primer pair targeting TMPRSS2 exon-1 and ERG exon-4. To distinguish induced endogenous fusion RNAs from exogenous input RNAs, mutations were introduced at nucleotide positions 16-35 of the input RNAs (gray box in TMPRSS2 exon-1), allowing specific amplifications of induced endogenous fusion RNAs or exogenous input RNA using specific forward primers. The lower panel shows the wild type TMPRSS2 exon-1 sequence in green. The mutated area in input RNA is shown in black and underlined. The primer used to specifically recognize induced fusion RNA is shown as the green arrow and was used in most of the figures in the main text. The primer for input RNA is denoted by the black arrow and was used in Fig. 1B, 1C, 1D, 1E, Fig. 2B, and Fig. 3B in the main text. 
A.
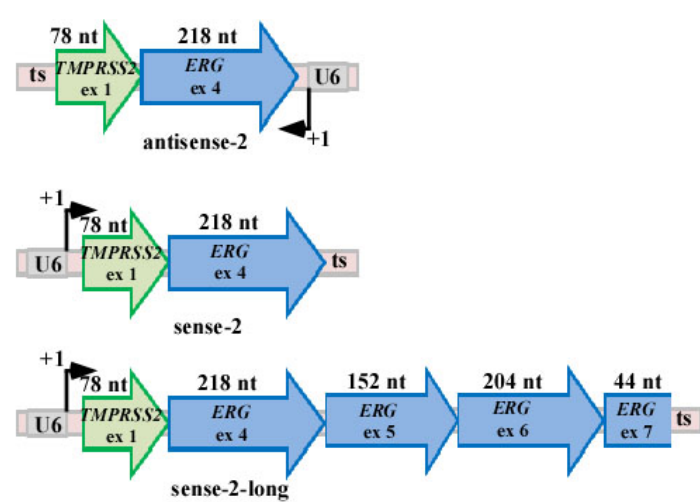

B.

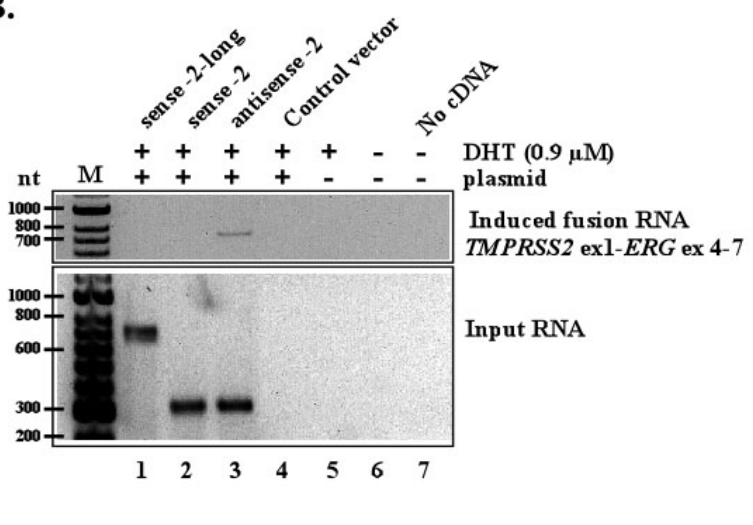

Fig. S2. Sense input RNAs that mimic trans-spliced products failed to induce fusion transcripts.

A. Schematics of the designed input RNAs consisting of TMPRSS2 (uc002yzj.3) and ERG (uc021wjd.1) exons. Upper panels: antisense-2 and sense-2 input RNA consisting of TMPRSS2 exon-1 (78 nt) and ERG exon-4 (216 nt). Lower panel: sense-2-long RNA consisting of TMPRSS2 exon-1 (78 nt) with ERG exons-4, -5, -6 and partial -7 (618 nt). ts: transcriptional stop "TTTTTT" for the U6 promoter. B. RT-PCR detection of induced fusion transcript (top gel) and input RNA (bottom gel). For induced fusion transcript, RT-PCR was performed using a forward primer on TMPRSS2 exon-1 paired with a reverse primer on the 3' end of ERG exon-7 (see Fig. S1A), thereby specifically amplifying the endogenous fusion RNA transcribed from the TMPRSS2-ERG fusion gene, but not from transfected plasmids or input RNAs because both lack the 3' end of ERG exon-7. Control vector lacking input RNA sequence (lane 4), DHT treatment only (lane 5), and PCR reactions lacking cDNA (lane 7) were used as negative controls. Antisense RNA is capable of inducing the fusion transcript (lane 3), whereas sense RNAs failed to induce fusion transcripts regardless of their lengths (lanes 1 and 2). 
bioRxiv preprint doi: https://doi.org/10.1101/151241: this version posted June 16, 2017. The copyright holder for this preprint (which was not certified by peer review) is the author/funder, who has granted bioRxiv a license to display the preprint in perpetuity. It is made available under aCC-BY 4.0 International license.
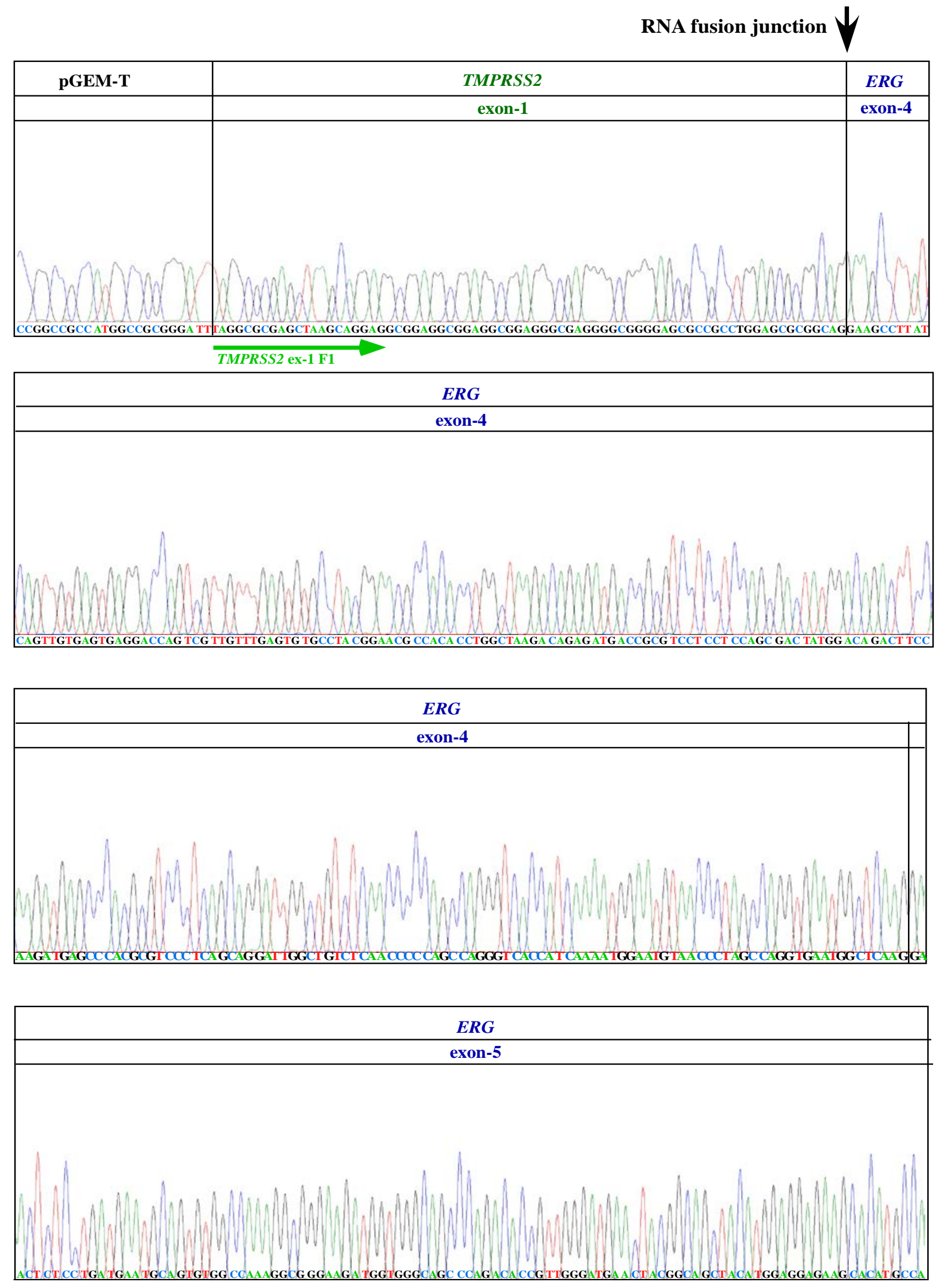
bioRxiv preprint doi: https://doi.org/10.1101/151241; this version posted June 16, 2017. The copyright holder for this preprint (which was not certified by peer review) is the author/funder, who has granted bioRxiv a license to display the preprint in perpetuity. It is made available under aCC-BY 4.0 International license.
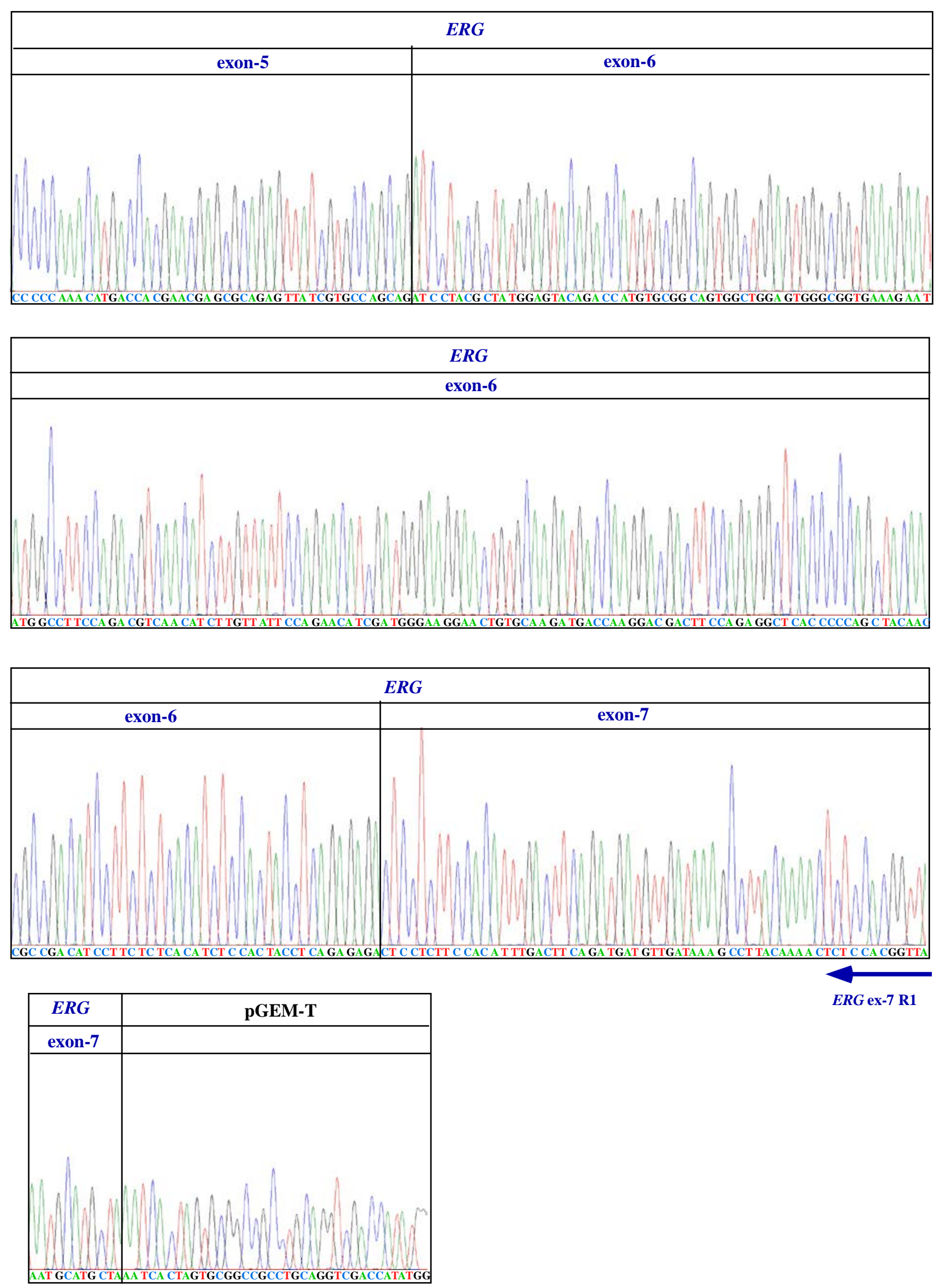

ERG ex-7 R1

ERG ex-7 R1 
Fig. S3. Sanger sequencing confirmation of the induced TMPRSS2-ERG fusion transcript in LNCaP cells mediated by antisense- 2 input RNA.

Induced fusion transcripts were converted to cDNA using oligo dT primers and PCR was performed using primers targeting TMPRSS2 exon-1 and ERG exon-7 as described in Fig. S1. The RT-PCR assay relies on the primer targeting ERG exon-7 to specifically amplify the induced fusion transcript but not the input RNA, which lacks ERG exon-7 sequence. The locations of the primers used to amplify the PCR product are shown as the green and blue arrows for TMPRSS2 exon-1 and ERG exon-7, respectively. The RNA fusion junction between TMPRSS2 exon-1 and ERG exon-4 is indicated by the black arrow. The pGEM-T plasmid was used for cloning the cDNA inserts. Sanger sequencing chromatograms confirmed that the induced fusion transcript contains TMPRSS2 exon-1 fused to ERG exons-4, $-5,-6$, and -7 , and that these exons were joined by splicing at the annotated splice sites as would be expected of mature endogenous TMPRSS2ERG fusion mRNA. This result confirms that expression of an input RNA can lead to specific fusion transcript in human cells. 
bioRxiv preprint doi: https://doi.org/101101/151241. this version posted June 16,2017. The copyright holder for this preprint (which was not certified by peer review) is the author/funder, who has granted bioRxiv a license to display the preprint in perpetuity. It is made available under aCC-BY 4.0 International license.
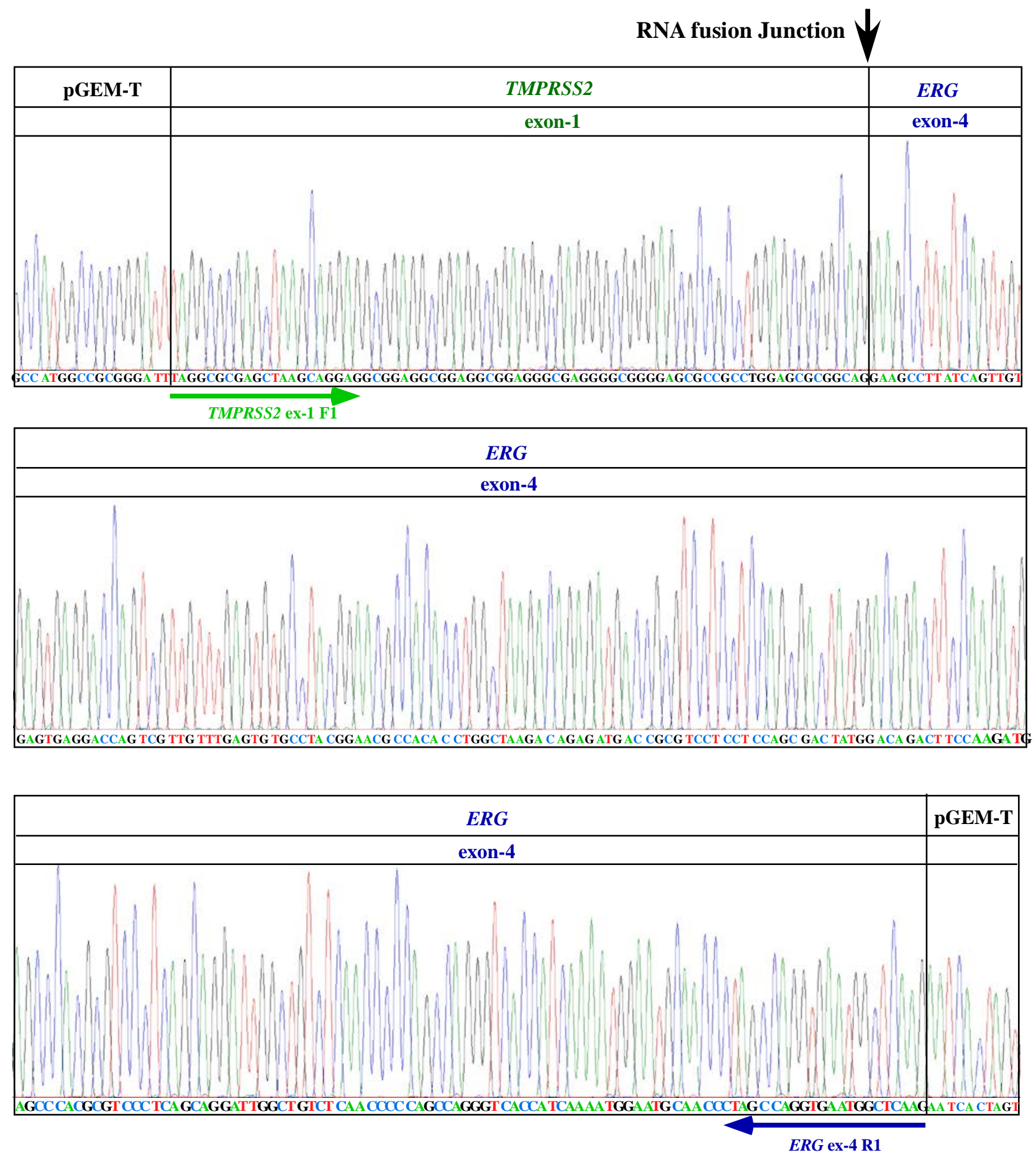

Fig. S4. Sanger sequencing confirmation of the induced TMPRSS2-ERG fusion transcripts mediated by antisense-5 input RNA and amplified using an optimized primer pair.

Induced fusion transcripts were converted to cDNA using oligo dT primers. RT-PCR was performed with primers targeting TMPRSS2 exon-1 and ERG exon-4, which led to higher 
amplification efficiency. This RT-PCR assay relies on the primer targeting TMPRSS2 exon-1 to specifically amplify the induced fusion transcript but not the input RNA, which contains the mutated region described in Fig S1B. The locations of the primers are shown as green and blue arrows for TMPRSS2 exon-1 and ERG exon-4, respectively. The RNA fusion junction between TMPRSS2 exon-1 and ERG exon-4 is indicated by the black arrow. The pGEM-T plasmid was used for cloning the cDNA inserts. Sanger sequencing chromatograms confirmed that the induced fusion transcript contained TMPRSS2 exon-1 fused to ERG exon-4, as would be expected of mature endogenous TMPRSS2-ERG fusion mRNA. We developed this primer pair to optimize the efficiency of detecting low levels of induced endogenous TMPRSS2-ERG fusion mRNA, and these primers were used for most of the RT-PCR assays. 

aCC-BY 4.0 International license.
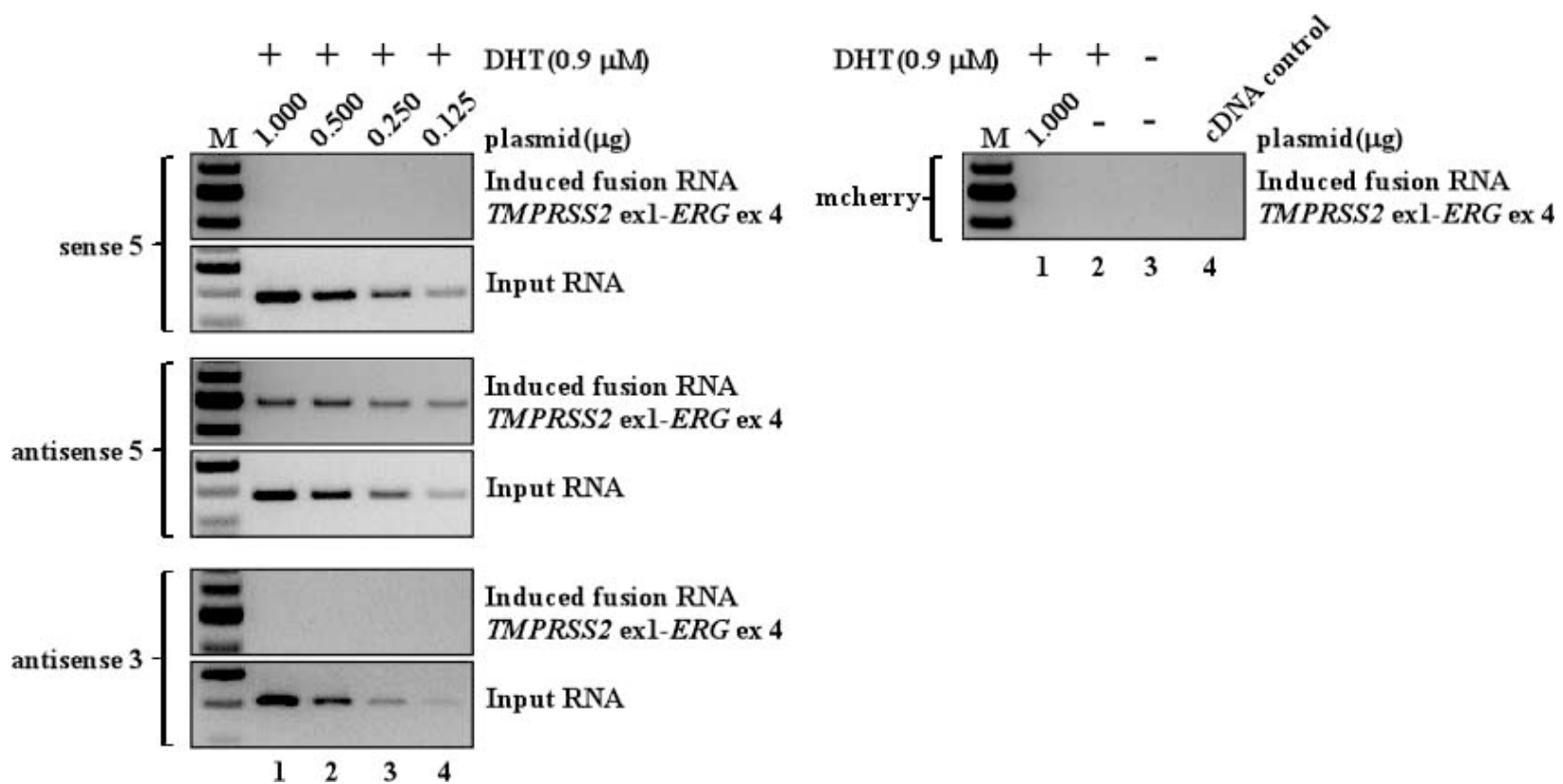

Fig. S5. Antisense-5 but not its corresponding sense-5 induces TMPRSS2-ERG gene-fusion.

Different amount of plasmids $(1.000 \mu \mathrm{g}, 0.500 \mu \mathrm{g}, 0.250 \mu \mathrm{g}$ and $0.125 \mu \mathrm{g})$ expressing either sense5 or antisense- 5 were transfected to produce different amount of corresponding input RNAs in LNCaP cells. To maintain transfection efficiency, mCherry control plasmid was added to each transfection to make the final amount of plasmid to $1.0 \mu \mathrm{g}$. Induction was done for three days in the presence of $0.9 \mu \mathrm{M}$ DHT. RT-PCR was performed to detect the levels of both induced TMPRSS2-ERG transcript and input RNA. RT-PCR results shows that even very low amount of antisense-5 input RNA is sufficient to induce TMPRSS2-ERG gene fusion (middle panel, lane 4) whereas very high level of sense-5 input RNA fails to induce TMPRSS2-ERG gene fusion (upper panel, lane 1). Thus, it is not the amount of the input RNA but the orientation of the input RNA (antisense vs. sense) that is important for fusion induction. Antisense-3, which is unable to induce fusion, was used as a negative control. mCherry alone was also transfected as a negative control. Additional controls (right panel): a vector expressing mCherry lacking input RNA sequence (lane 1), DHT treatment only (lane 2), and PCR reaction lacking cDNA (lane 4). 
A.
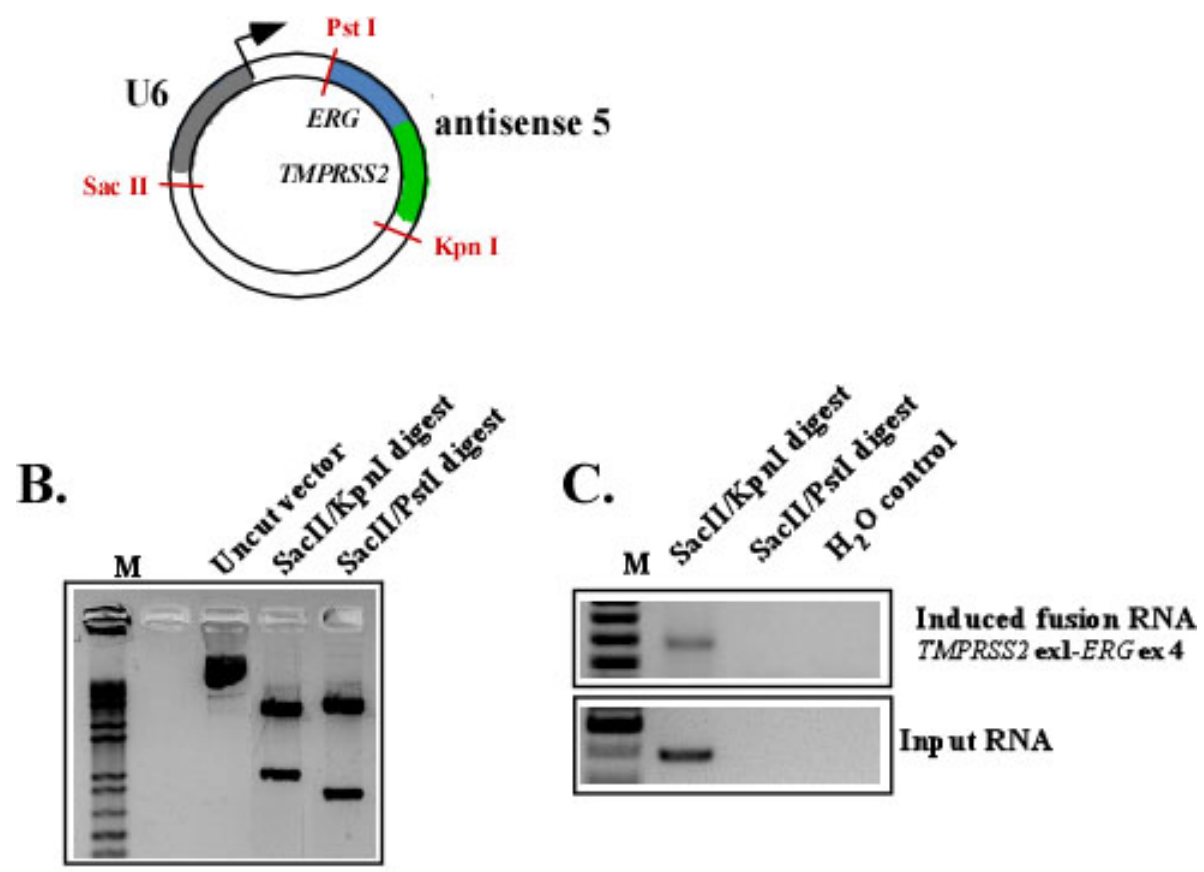

Digested DNA fragments

Fig. S6. Expressed input RNA, but not the DNA plasmid, induces fusion transcript.

A. Schematic of the expression plasmid containing a U6 promoter and antisense-5 sequence. Unique restriction sites are shown in red. The arrow indicates the transcriptional start. The plasmid was digested by SacII/KpnI, leaving U6 with antisense-5, or with SacII/PstI, separating U6 from antisense-5. B. Confirmation of complete digestion was visualized by agarose gel. Uncut plasmid vector was used as control. C. Induction of TMPRSS2-ERG fusion after transfection of digested plasmid fragments. The TMPRSS2-ERG fusion transcript was induced only when the U6 promoter was attached to antisense-5 sequence, indicating that it is the input RNA expressed by the U6 promoter, not the transfected DNA plasmid per se, that leads to fusion induction. 


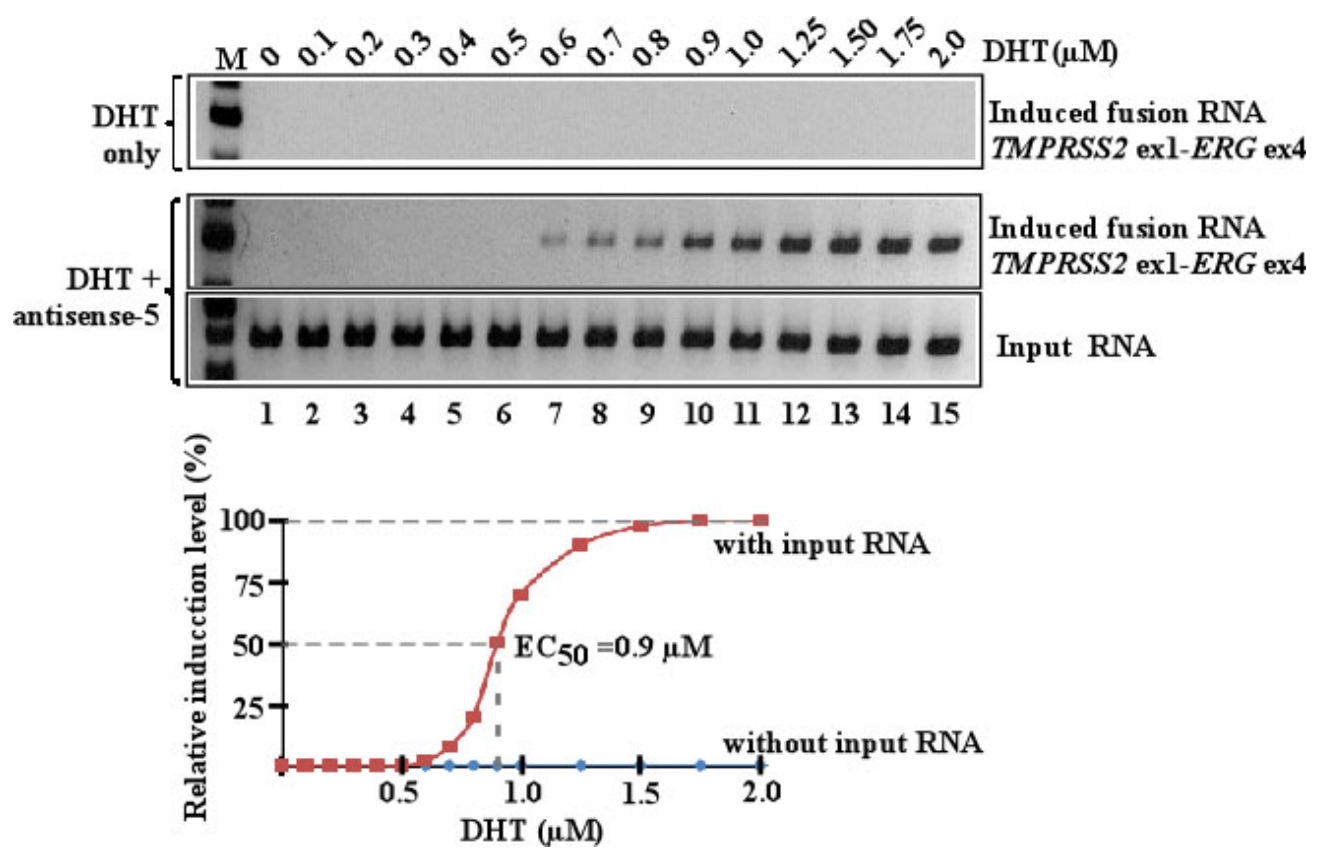

Fig. S7. DHT dosage effect in facilitating RNA-mediated gene fusion as assayed by one round RT-PCR.

To determine the DHT concentrations that facilitate RNA-mediated gene fusion detectable by one round RT-PCR, we generated a dose-response curve using LNCaP cells transfected with antisense-5 and treated with different DHT concentrations for 3 days. Relative RT-PCR intensities of induced fusion transcript bands as generated by one round RT-PCR were plotted against DHT concentrations. In the presence of antisense-5, fusion induction is detectable at $\sim 0.6$ $\mu \mathrm{M}$ DHT, reaches its maximum at $1.5 \mu \mathrm{M}$ DHT, with the effective concentration that yields $50 \%$ of maximal induction $\left(\mathrm{EC}_{50}\right)$ at $\sim 0.9 \mu \mathrm{M}$. In the absence of antisense-5, no induction was observed in the entire DHT range tested up to $2.0 \mu \mathrm{M}$ (upper panel), indicating that DHT alone is ineffective in inducing TMPRSS2-ERG fusion. To streamline our experimental procedures, we used $0.9 \mu \mathrm{M}$ DHT (the $\mathrm{EC}_{50}$ concentration) for 3 days followed by one round of RT-PCR as our standard assay in the main text. However, it is important to note that all key experiments were also performed under physiologically relevant DHT concentrations $(<100 \mathrm{nM})$, and in those cases three-rounds of nested PCR were used to reveal the lowest amount of DHT required. As shown in Fig. 1D and Fig. 6A in the main text, gene fusion events induced by antisense-5 occurred at physiologically relevant DHT concentrations as low as $20 \mathrm{nM}$, and gene fusion events induced by endogenous AZI1 mRNA occurred at as low as $40 \mathrm{nM}$ DHT. 


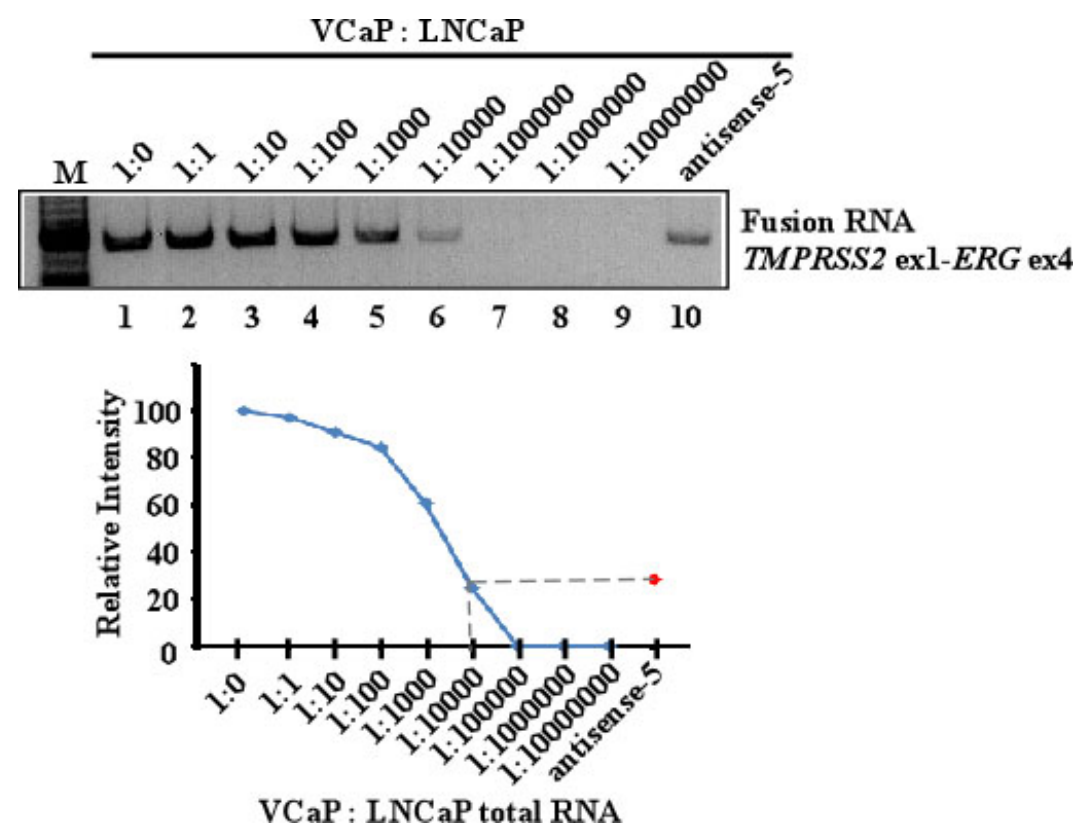

Fig. S8. Estimation of the percentage of LNCaP cells transfected with antisense-5 that carry the induced TMPRSS2-ERG fusion.

To estimate the percentage of LNCaP cells that underwent TMPRSS2-ERG gene fusion mediated by antisense- 5 at $0.9 \mu \mathrm{M}$ DHT (the $\mathrm{EC}_{50}$ ), we mixed various amounts of total RNA from VCaP cells that express TMPRSS2-ERG (Mertz et al., 2007; Teles Alves et al., 2013) with the total RNA from untransfected LNCaP cells in ratios of 1:1 up to 1:107. A standard dilution curve was determined using the relative RT-PCR intensity of the TMPRSS2-ERG fusion transcript. The relative intensity of induction by antisense- 5 in LNCaP cells, as assayed under the same RT-PCR conditions, is shown as a red dot that corresponds to an equivalent dilution of $1: 10^{3}$ to $1: 10^{4}$, suggesting that approximately 1 in $10^{3}$ or $10^{4}$ LNCaP cells is positive of TMPRSS2-ERG fusion. 


\begin{tabular}{|c|c|c|c|}
\hline & TMPRSS2 (-strand) & $E R G$ (-strand) & Tm \\
\hline \multicolumn{4}{|l|}{ STEMS } \\
\hline A & chr21:41508022-41508035 & chr21:38445526-38445539 & $44^{\circ} \mathrm{C}$ \\
\hline B & $\operatorname{chr} 21: 41508030-41508040$ & chr21:38463727-38463737 & $40^{\circ} \mathrm{C}$ \\
\hline $\mathrm{C}$ & $\operatorname{chr} 21: 41507658-41507669$ & $\operatorname{chr} 21: 38457001-38457012$ & $40^{\circ} \mathrm{C}$ \\
\hline D & $\operatorname{chr} 21: 41507876-41507888$ & chr21:38451230-38451242 & $44^{\circ} \mathrm{C}$ \\
\hline E & chr21:41499266-41499277 & chr21:38481641-38481652 & $30^{\circ} \mathrm{C}$ \\
\hline F & chr21:41507207-41507213 & chr21:38482731-38482737 & $24^{\circ} \mathrm{C}$ \\
\hline G & chr21:41503846-41503852 & chr21:38455751-38455757 & $16^{\circ} \mathrm{C}$ \\
\hline \multicolumn{4}{|l|}{ EXONS } \\
\hline TMPRSS 2 exon-1 & $\operatorname{chr} 21: 41508081-41508158$ & & \\
\hline TMPRSS2 exon-2 & chr21:41498119-41498189 & & \\
\hline$E R G$ exon-3 & & $\operatorname{chr} 21: 38575662-38575747$ & \\
\hline$E R G$ exon- 4 & & chr21:38445404-38445621 & \\
\hline
\end{tabular}

Fig. S9. Genome coordinates and Tm for the putative genomic stems described in Fig. 2C and $2 \mathrm{D}$ of the main text.

Genome coordinates are based on the GRCh38/hg38 version. Coordinates of the exons (TMPRSS2 exon-1 and -2; ERG exon-3 and -4) are also shown. 


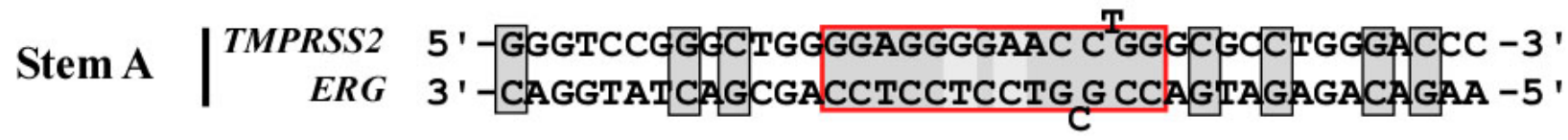

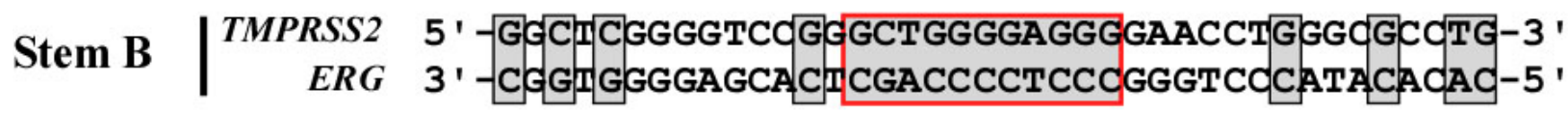

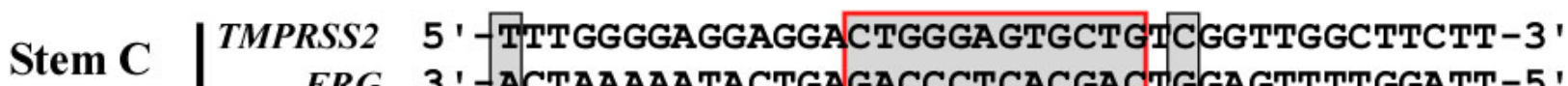
ERG 3 '-ACTAAAAATACTGAGACCCTCACGACTGGAGTTTTGGATT-5'

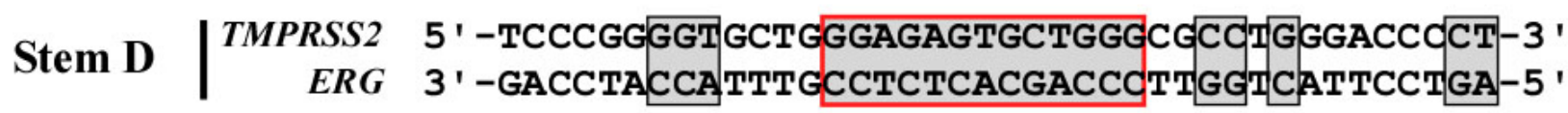

Stem E | TMPRSS2 5'-GTTGACGGCATCTCTAGCTATTATTGTTAACTGTTCACAA-3' ERG 3'-ATGTTTTTGTTTGCATCGATAATAACTTTTACAATACGAT-5 '

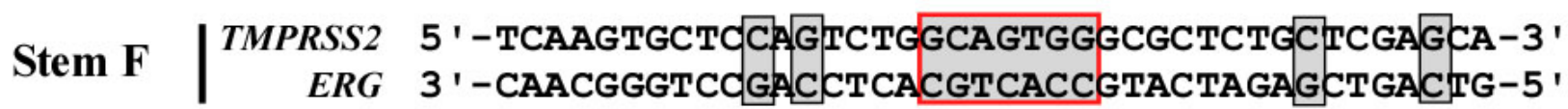

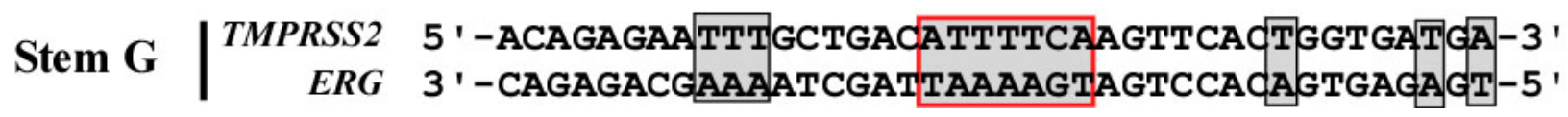

Fig. S10. Complementary base pairing of the genomic DNA sequences comprising the stems and their flanking sequences.

The sense strand of genomic sequences from TMPRSS2 and ERG that form the putative stems (in red boxes) with various degrees of stability as identified by BLAST analyses. Regions showing perfect complementary base pairing (A-T and G-C) are shaded in gray. 


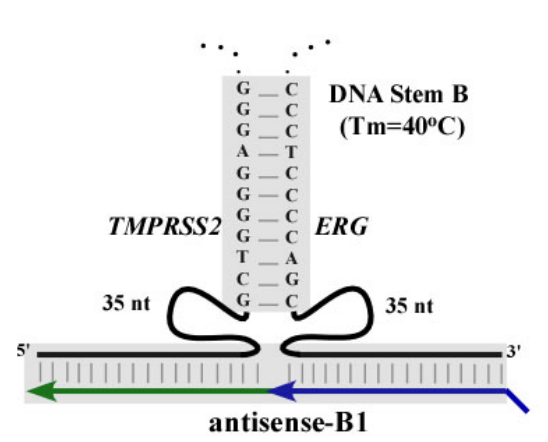

antisense-B1

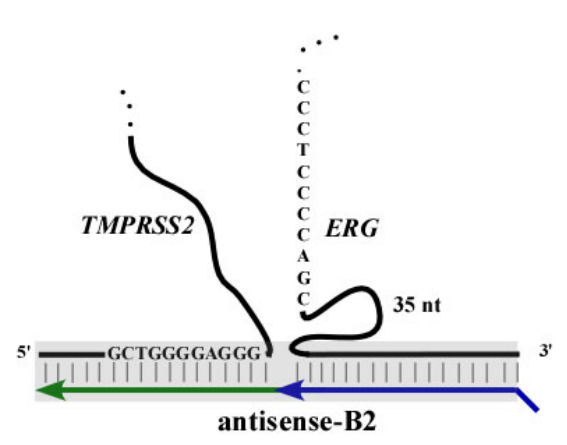

antisense-B2
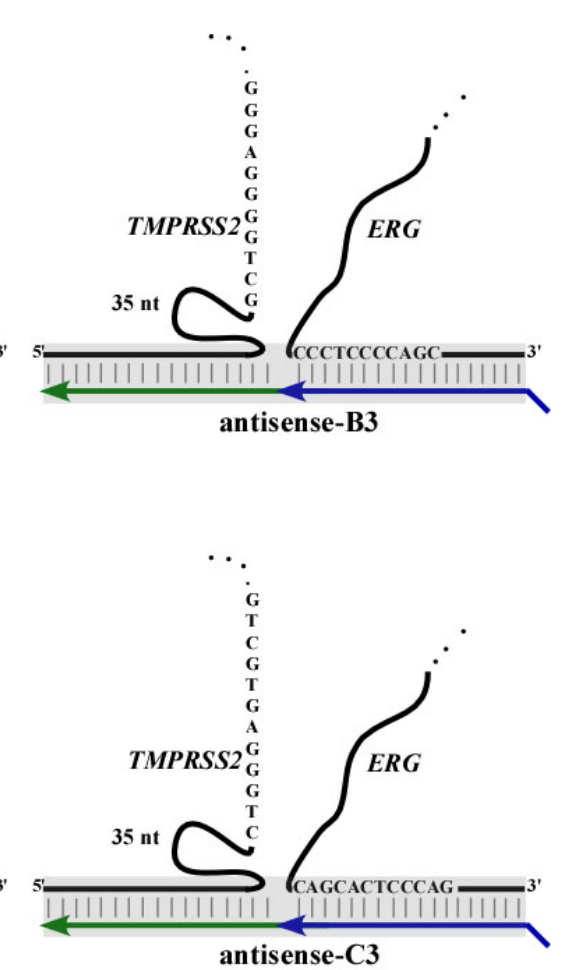

antisense-C2
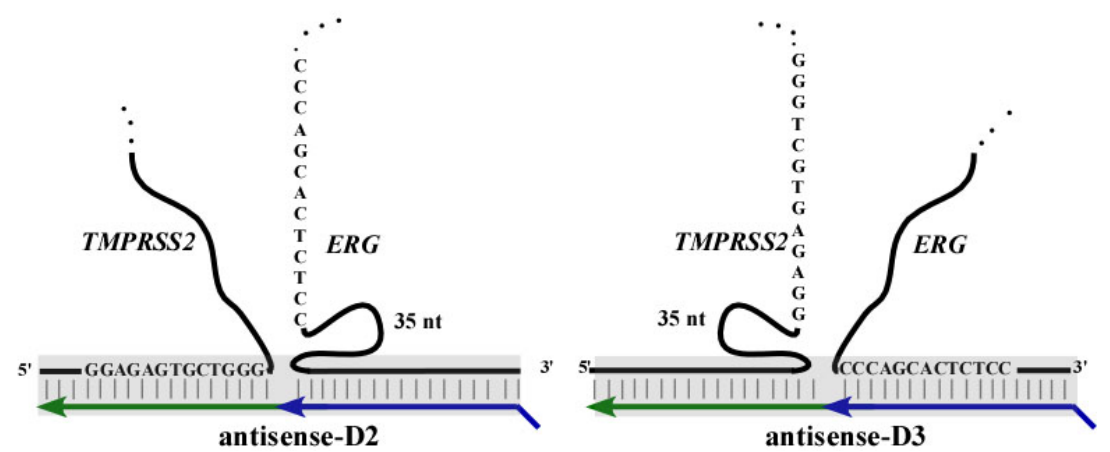

Fig. S11. Antisense input RNAs designed to disrupt the genomic stems B, C, and D.

Stem B (upper row). Stem C (middle row). Stem D (lower row). Left column: Illustrations show the three-way junction formed by genomic DNA and corresponding antisense input RNA (antisense-B1, C1, and D1). Middle column: Disruption of stems via the TMPRSS2 side using tailor-made input RNAs (antisense-B2, C2, and D2) that directly hybridize to the TMPRSS2 stem sequence. Right column: Disruption of stems via the $E R G$ side using tailor-made input RNAs (antisense-B3, C3, and D3) that directly hybridize to the ERG stem sequence. Shaded regions indicate base pairing. The TMPRSS2 portion of the input RNA is shown in green and the ERG portion is in blue. 
A.

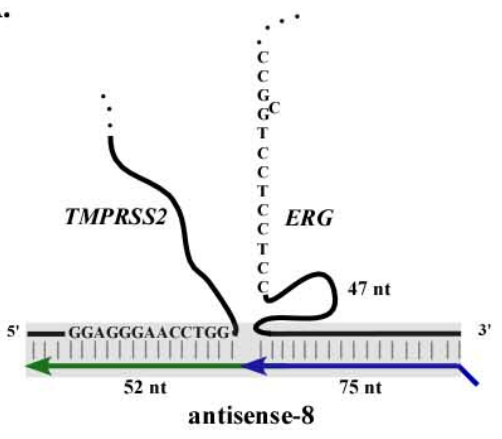

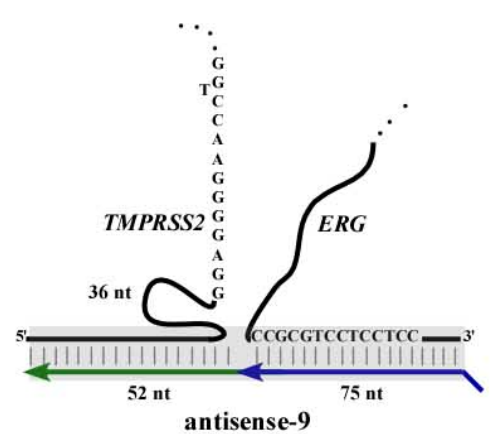

B.

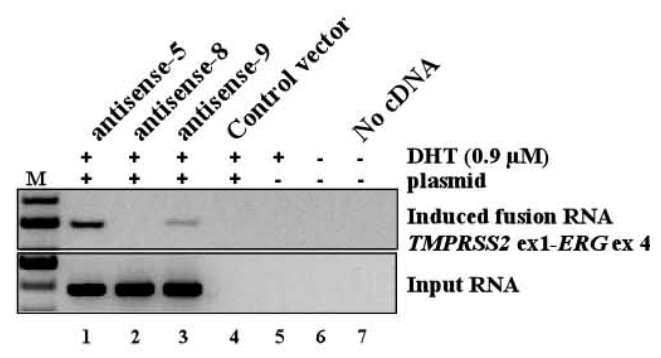

Fig. S12. Antisense input RNAs designed to disrupt the genomic stems A.

A. Disruption of the putative genomic stem using tailor-made input RNAs that directly hybridize to one side of the stem A. B. RT-PCR assays of fusion transcripts induced by the input RNAs illustrated in A. Interfering with three-way junction formation eliminated (lane 2) or significantly reduced the induction (lane 3). Controls include: vector lacking input RNA sequence (lane 4), DHT treatment only (lane 5), and PCR reaction lacking cDNA (lane 7). 
A.

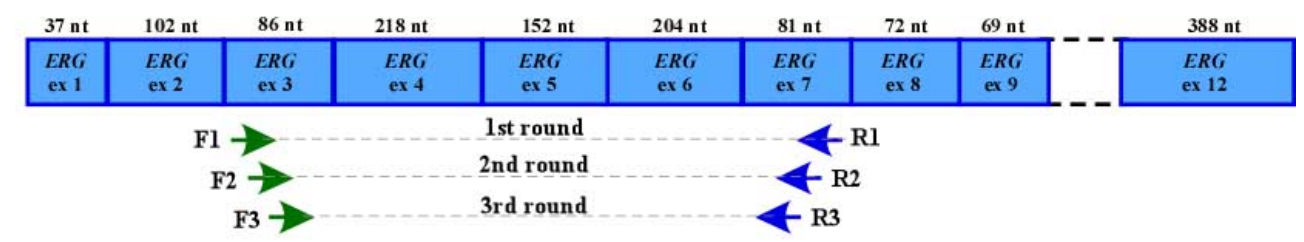

B.

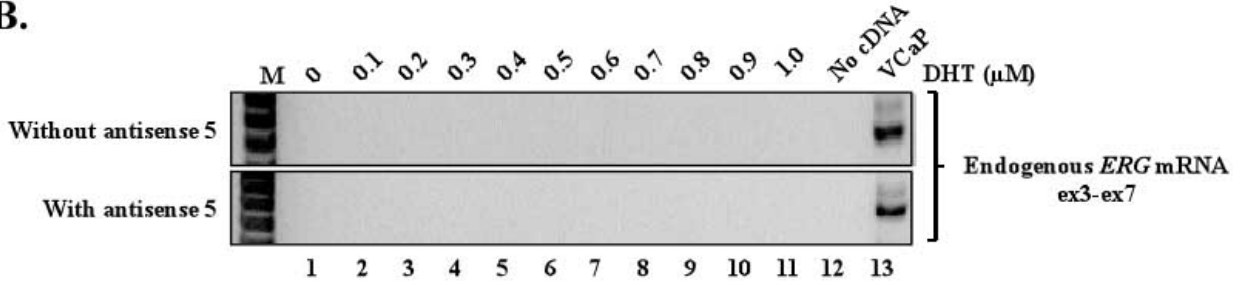

C.
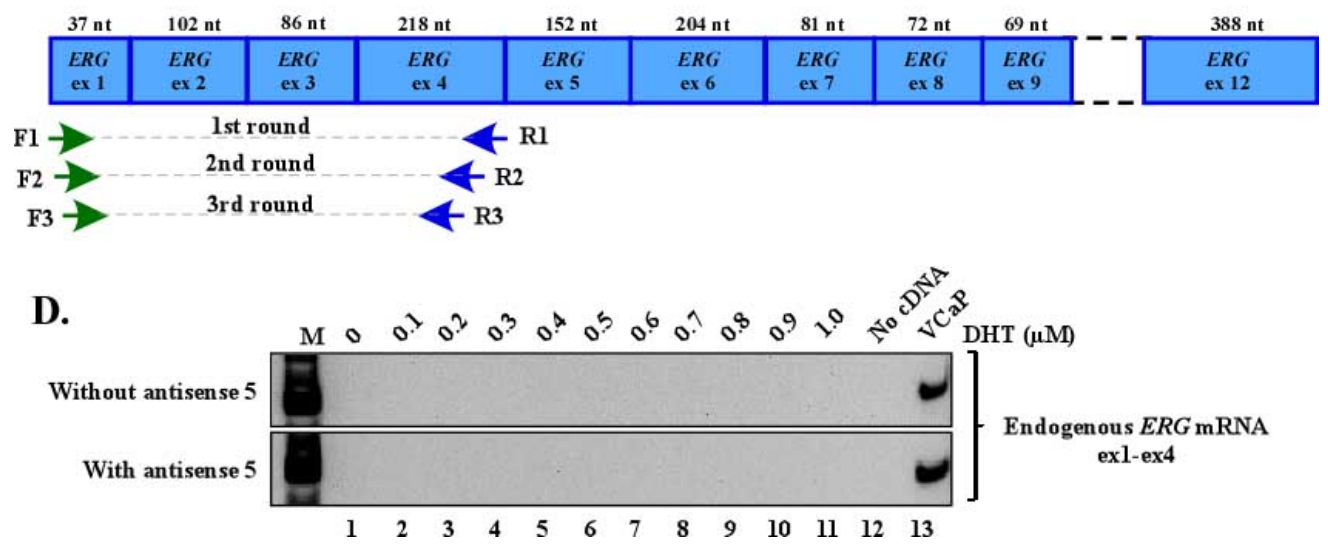

\section{Fig. S13. Endogenous ERG mRNA is not detected in LNCaP cells by three-rounds of nested RT-PCR.}

A. Nested primer pairs used to detect low levels of endogenous ERG mRNA from LNCaP cells. Forward primers for ERG exon-3 are shown in green whereas reverse primers for ERG exon-7 are shown in blue (primer sequences are listed in the Materials and Methods). B. Endogenous ERG mRNA was not detected in LNCaP cells in the presence or absence of DHT or antisense-5 (lane 1 to 11 ) by three-rounds of nested RT-PCR. cDNA prepared from VCaP cells which express ERG mRNA was used as positive control (lane 13). C and D. Similarly, using forward primers on ERG exon-1 and reverse primers on ERG exon-4 also failed to detect endogenous ERG mRNA by three-rounds of nested RT-PCR (lane 1 to 11, primer sequences are listed in the Materials and Methods). These primer sets were chosen because they specifically amply endogenous ERG mRNA but not TMPRSS2-ERG fusion transcript that contains ERG exon-3 to exon-12. 


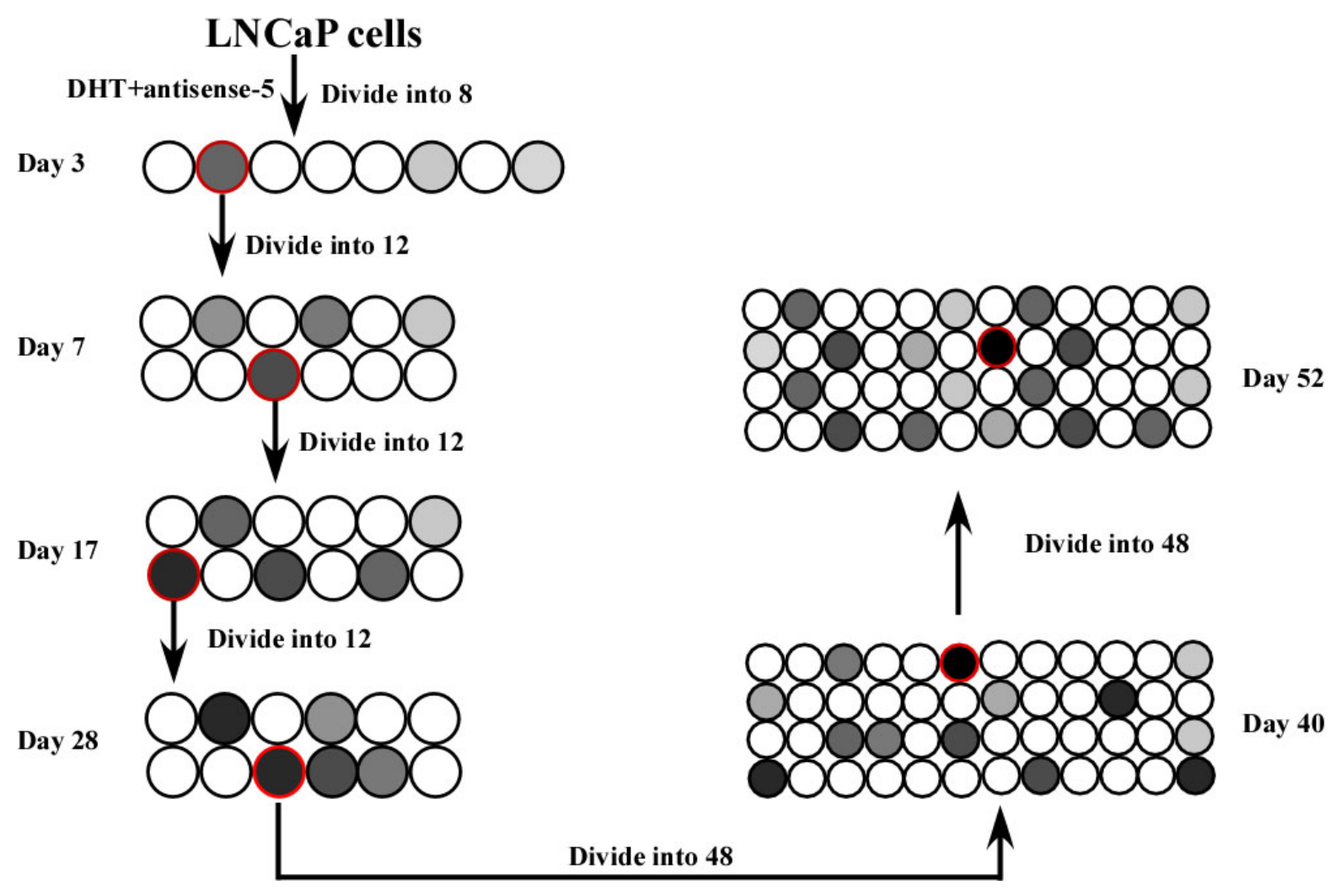

Fig. S14. Procedure used to propagate and enrich the induced LNCaP population for 52 days in the absence of DHT.

LNCaP cells were transfected on day 1 with antisense-5 and incubated for 3 days with DHT. Cells were then divided into sub-populations. Half of the cells from each sub-population were harvested for RT-PCR assays to detect the induced fusion transcript whereas the other half was used to propagate the population. The sub-population containing the highest intensity of induced fusion transcripts (circled in red) was selected for continuous propagation for the next round of division and RT-PCR assays. The process was continued for 52 days. RT-PCR was performed to detect both induced fusion transcripts and antisense input RNA during each division as shown in Fig. 3B. 
A.

Locations of 44 forward primers in TMPRSS2 intron-1

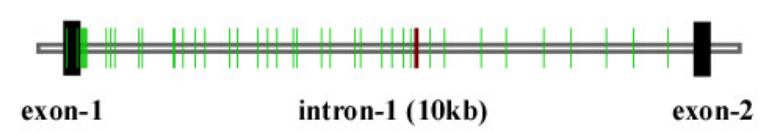

Locations of 112 reverse primers in ERG intron-3

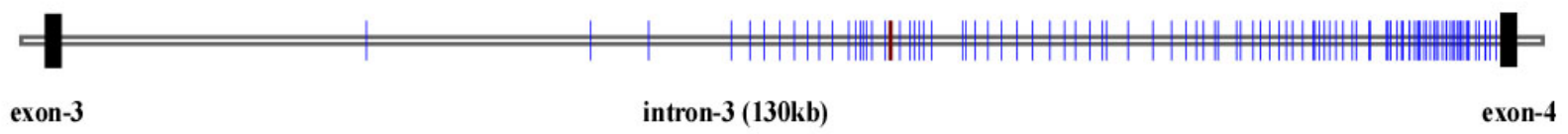

B.

Identified genomic breakpoints within TMPRSS2 intron-1

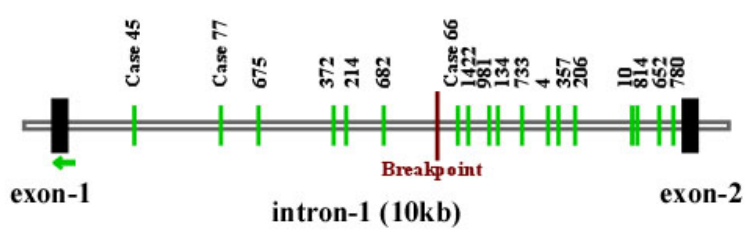

Identified genomic breakpoints with in $E R G$ intron-3

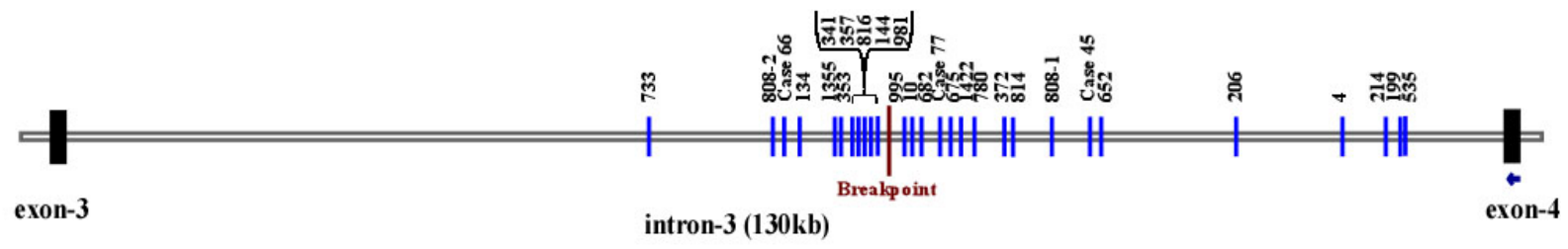

Fig. S15. Locations targeted by primers for mapping the genomic breakpoint and locations of identified TMPRSS2-ERG genomic breakpoints.

A. The locations of 44 forward primers (green) spacing across TMPRSS2 intron-1 ( 10 kb) and 112 reverse primers (blue) spacing across $E R G$ intron-3 ( 130 kb). ERG intron-3 is significantly larger than that of TMPRSS2 intron-1. Each vertical line represents a target location by a primer or a set of nested primers. The primers targeting ERG intron-3 are designed to concentrate in the region near exon-4 and in the hot spot regions previously identified from prostate cancer patients. The specific primers that amplify the genomic breakpoint shown in Fig. 3D in the main text are labeled as red. B. Locations of identified TMPRSS2-ERG genomic breakpoints. 
Locations of previously identified TMPRSS2-ERG genomic breakpoints from prostate cancer patients (Weier et al.) are shown in green in TMPRSS2 intron-1 and blue in ERG intron-3. The number on top of each location is the ID number of a patient. The genomic breakpoint identified in our study is shown in red. Short arrows indicate sites targeted by antisense- 5 . 
bioRxiv preprint doi: https://doi.org/10.1101/151241: this version posted June 16, 2017. The copyright holder for this preprint (which was not certified by peer review) is the author/funder, who has granted bioRxiv a license to display the preprint in perpetuity. It is made available under aCC-BY 4.0 International license.
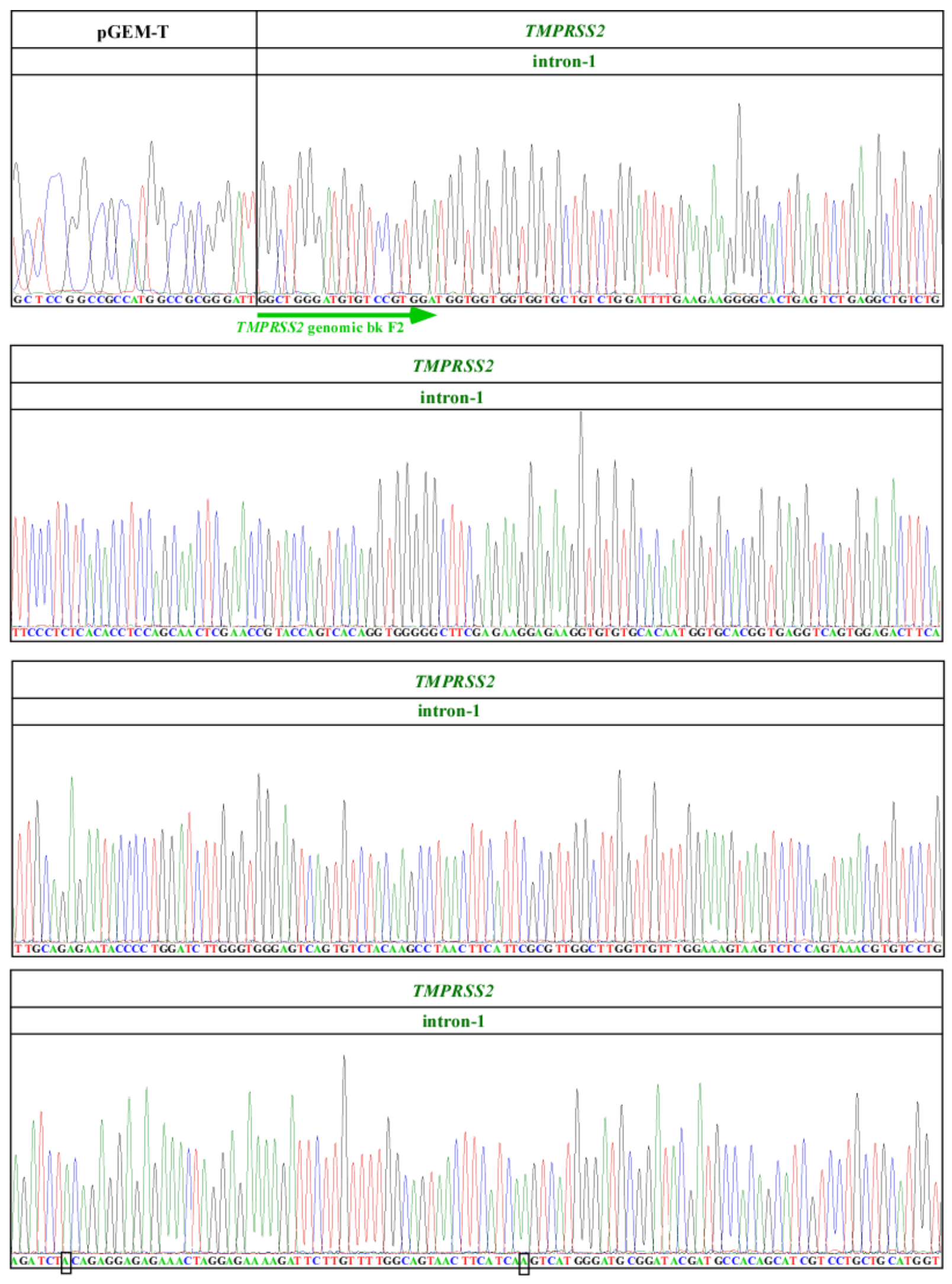
bioRxiv preprint doi: https://doi.org/10.1101/151241: this version posted June 16, 2017. The copyright holder for this preprint (which was not certified by peer review) is the author/funder, who has granted bioRxiv a license to display the preprint in perpetuity. It is made available under aCC-BY 4.0 International license.
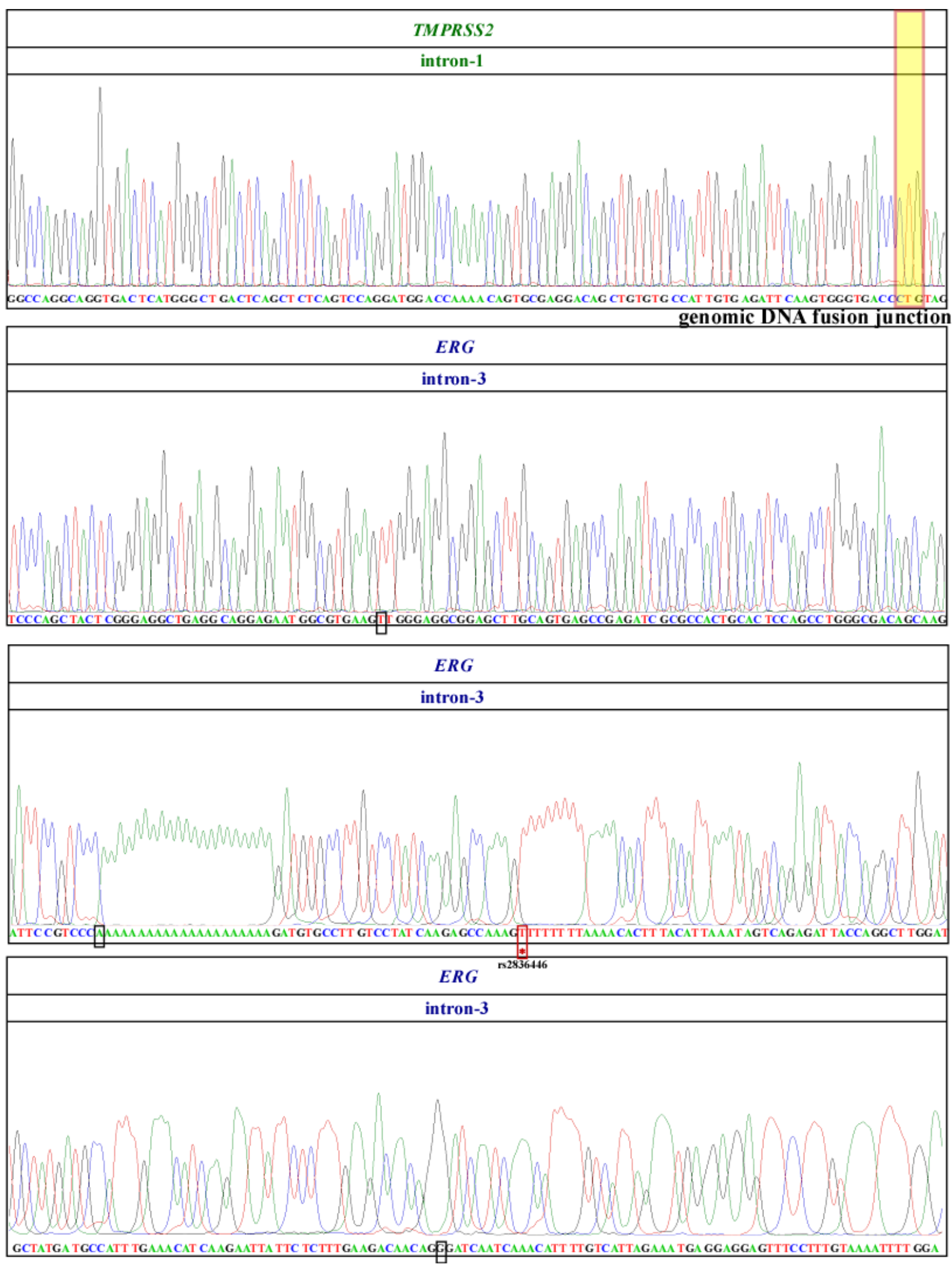


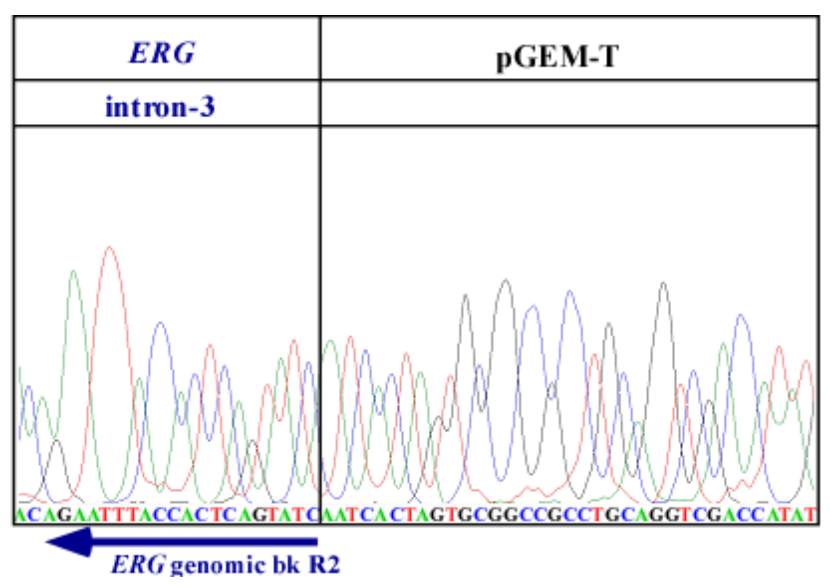

Fig. S16. Sanger sequencing confirmed that the induced TMPRSS2-ERG fusion is due to gene fusion resulting from genomic arrangement.

LNCaP cells transfected with antisense-5 were enriched for the TMPRSS2-ERG fusion transcript as described in Fig. S14. Genomic DNA PCR was performed on the enriched LNCaP population which yielded a gene fusion band of $\sim 862$ bp as shown in Fig. 3D in the main text. Sanger sequencing chromatograms confirmed that the 862 bp band contains 500 bp of TMPRSS2 intron-1 joined to $\sim 362$ bp of ERG intron-3. The locations of the primers used to amplify the PCR product are shown as the green and blue arrows for TMPRSS2 intron-1 and ERG intron-3, respectively under the chromatograms. The genomic breakpoint between TMPRSS2 intron-1 and $E R G$ intron-3 is indicated by the yellow shaded region that contains a three nucleotide 'CTG' microhomology. The pGEM-T plasmid was used for cloning the genomic DNA PCR product. Five mutations shown in black boxes are PCR artifacts because they are absent in other clones that were sequenced in parallel. The mutation denoted by the asterisk $(*)$ in the red box is present in all sequenced clones and is a known Single Nucleotide Polymorphism (rs2836446). 


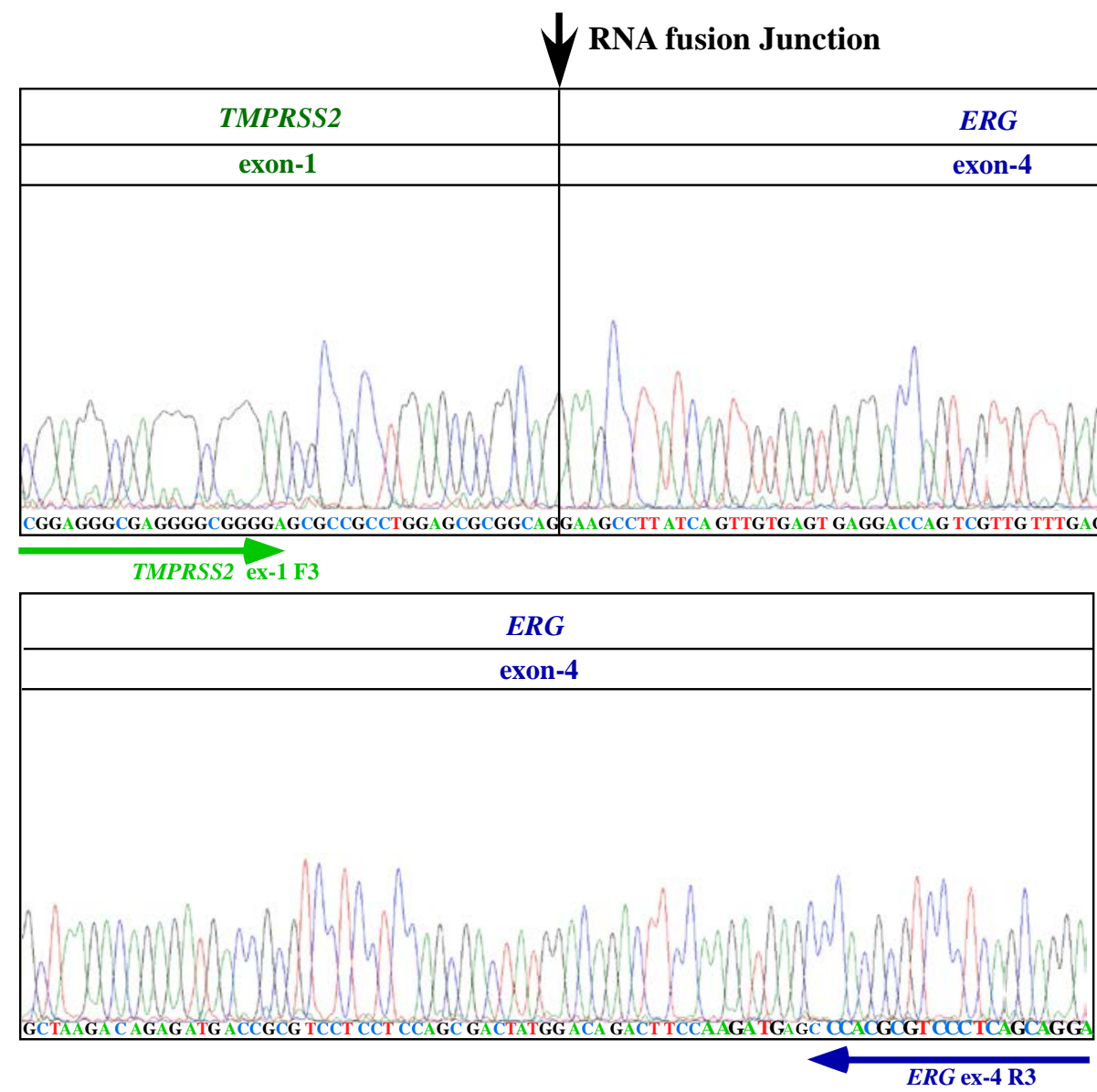

Fig. S17. Sanger sequencing confirmation of the induced TMPRSS2-ERG fusion transcript in PNT1A cells mediated by antisense-5 RNA.

Sanger sequencing confirmed that the induced fusion RNA in normal prostate epithelium cells (as shown in Fig. 3F in the main text) is the same fusion transcript containing TMPRSS2 exon-1 fused to ERG exon-4 as would be expected of mature endogenous TMPRSS2-ERG fusion mRNA. This indicates that induction of the TMPRSS2-ERG fusion transcript by input RNA is permissible in normal prostate epithelial cells prior to malignant transformation. The locations of the primers used to amplify the PCR product are shown as the green and blue arrows for TMPRSS2 exon-1 and ERG exon-4, respectively. The RNA fusion junction between TMPRSS2 exon-1 and ERG exon-4 is indicated by the black arrow. 

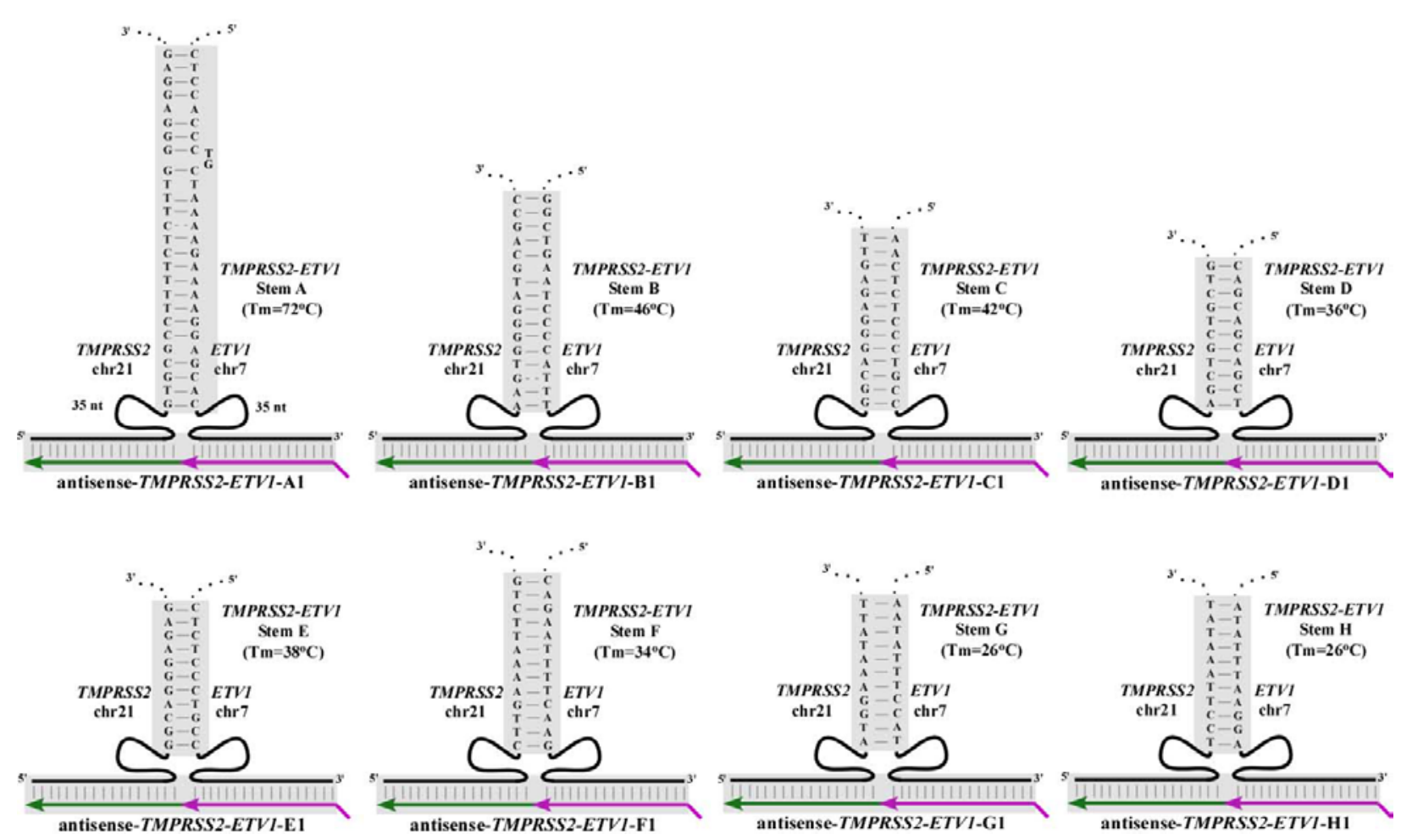

Fig. S18. Schematics of the putative three-way junctions formed between TMPRSS2 and ETV1 genomic DNA and the corresponding antisense input RNA.

We used BLAST alignment to identify eight stems that could be formed with varying degrees of stability by the sense genomic TMPRSS2 sequence paired with the sense genomic ETV1 sequence. Matching antisense RNAs were then designed to forge the three-way junction with these putative intron stems. Shaded regions indicate base pairing between antisense input RNA (in green and purple) and genomic DNA (in black). TMPRSS2 is on chromosome 21 whereas ETV1 is on chromosome 7. For both, the genomic minus strand is the sense strand. 


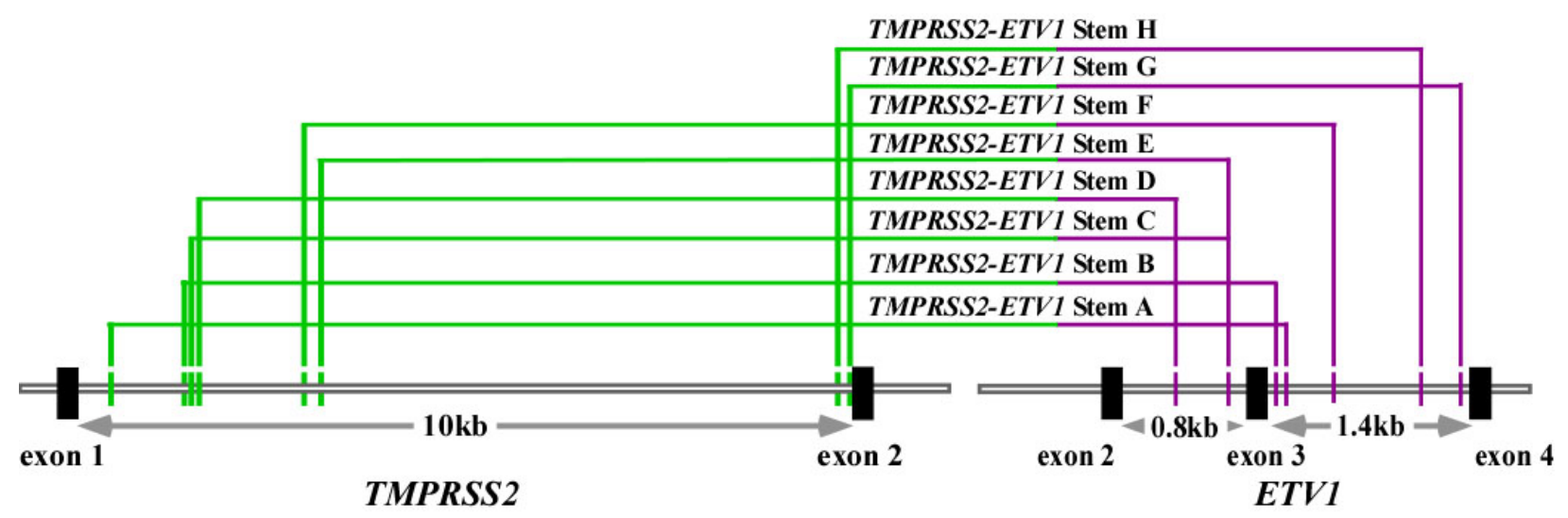

\begin{tabular}{|l|l|l|l|}
\hline TMPRSS2 (-strand) & ETV (-strand) & Tm \\
\hline TMPRSS2-ETV1 stem A & chr21:41507672-41507697 & chr7:13988013-13988040 & $72^{\circ} \mathrm{C}$ \\
\hline TMPRSS2-ETV1 stem B & chr21:41506698-41506713 & chr7:13988054-13988069 & $46^{\circ} \mathrm{C}$ \\
\hline TMPRSS2-ETV1 stem C & chr21:41506649-41506661 & chr7:13988373-13988385 & $42^{\circ} \mathrm{C}$ \\
\hline TMPRSS2-ETV1 stem D & chr21:41506584-41506594 & chr7:13988732-13988742 & $36^{\circ} \mathrm{C}$ \\
\hline TMPRSS2-ETV1 stem E & chr21:41505067-41505077 & chr7:13988373-13988383 & $38^{\circ} \mathrm{C}$ \\
\hline TMPRSS2-ETV1 stem F & chr21:41505211-41505223 & chr7:13987620-13987632 & $34^{\circ} \mathrm{C}$ \\
\hline TMPRSS2-ETV1 stem G & chr21:41498215-41498225 & chr7:13986643-13986753 & $26^{\circ} \mathrm{C}$ \\
\hline TMPRSS2-ETV1 stem H & chr21:41498359-41498369 & chr7:13986021-13987031 & $26^{\circ} \mathrm{C}$ \\
\hline EXONS & & & \\
\hline TMPRSS2 exon-1 & chr21:41508081-41508158 & & \\
\hline TMPRSS2 exon-2 & chr21:41498119-41498189 & & \\
\hline ETV1 exon-2 & & chr7:13989090-13989139 & \\
\hline ETV1 exon-3 & & chr7:13988140-13988173 & \\
\hline ETV1 exon-4 & & & \\
\hline
\end{tabular}

Fig. S19. Genome coordinates and Tm for the putative TMPRSS2-ETV1 genomic stems described in S18.

Upper panel: Locations of putative TMPRSS2-ETV1 stems A, B, C, D, E, F, G, and H identified by BLAST alignment. All stem sequences are located in TMPRSS2 intron-1 (chromosome 21) paired with sequences in ETV1 intron-2 or -3 (chromosome 7). Genome coordinates are based on GRCh38/hg38 version. Coordinates of the exons (TMPRSS2 exons-1 and -2; ETV1 exons-2, -3, and -4) are also shown. 
bioRxiv preprint doi: https://doi.org/10.1101/151241; this version posted June 16, 2017. The copyright holder for this preprint (which was not certified by peer review) is the author/funder, who has granted bioRxiv a license to display the preprint in perpetuity. It is made available under aCC-BY 4.0 International license.
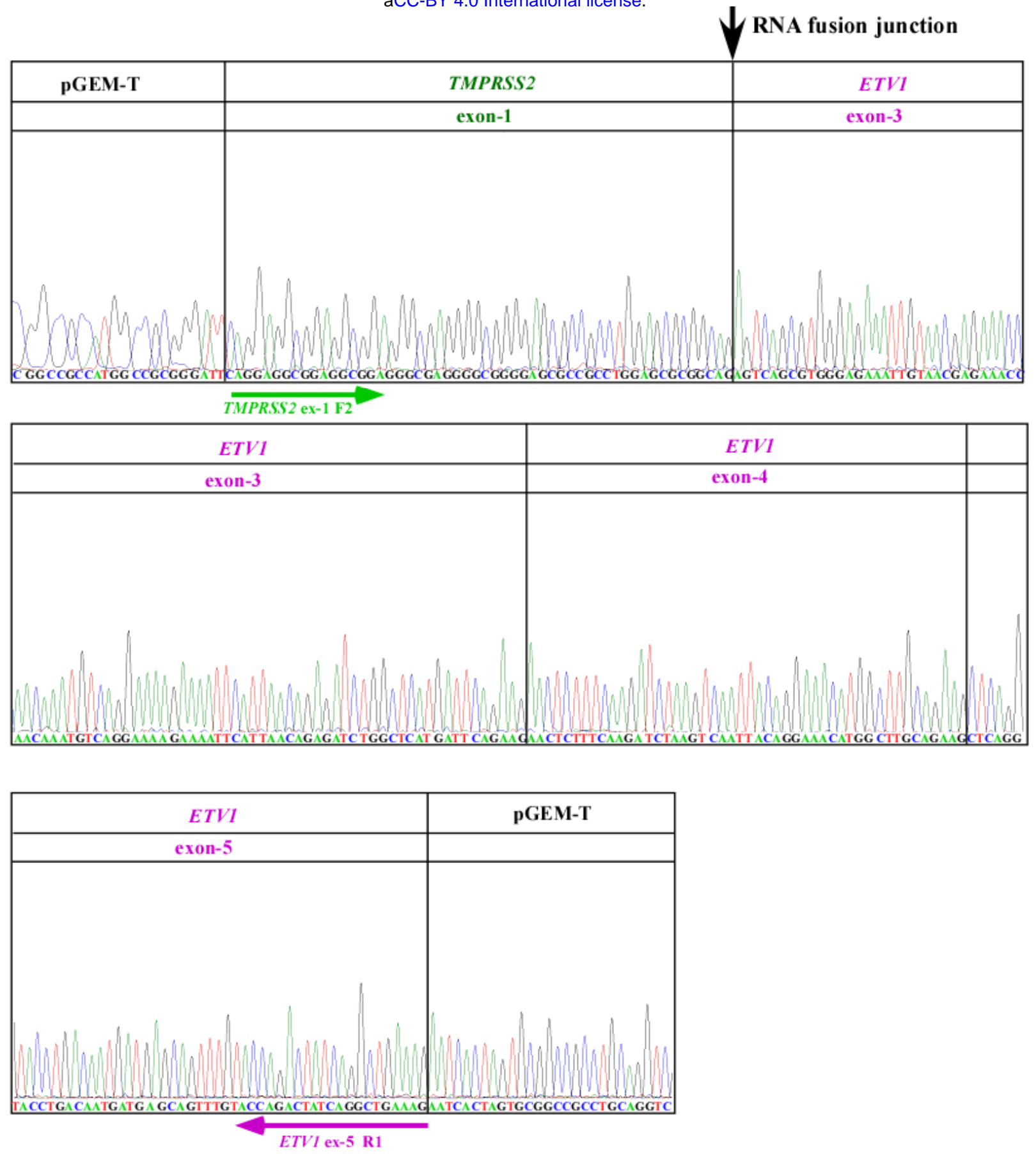

Fig. S20. Sanger sequencing confirmed the induced TMPRSS2-ETV1 fusion transcripts in LNCaP cells.

Sanger sequencing chromatograms confirmed that the induced RNA contains TMPRSS2 exon-1 fused to ETV1 exon-3 as would be expected of mature endogenous TMPRSS2-ETV1 fusion mRNA. These results suggest that RNA-mediated gene fusion in mammalian cells is not restricted to TMPRSS2 and ERG but appears to be generally permissible regardless of whether it is an intra-chromosomal (TMPRSS2-ERG) or inter-chromosomal (TMPRSS2-ETV1) fusion. The 
induced fusion transcript was converted to cDNA using oligo dT primers and PCR was performed using primers targeting TMPRSS2 exon-1 and ETV1 exon-5. The locations of the primers are shown as the green and purple arrow for TMPRSS2 exon-1 and ETV1 exon-5, respectively. The RNA fusion junction between TMPRSS2 exon-1 and ETV1 exon-3 is indicated by the black arrow. 


\section{Locations of 23 forward primers in TMPRSS2 intron-1}

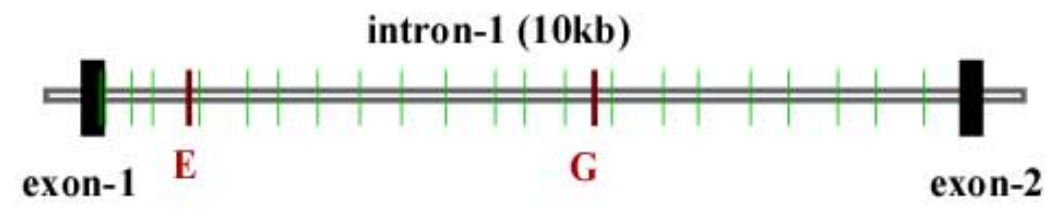

Locations of 7 reverse primers in ETV1 intron-2

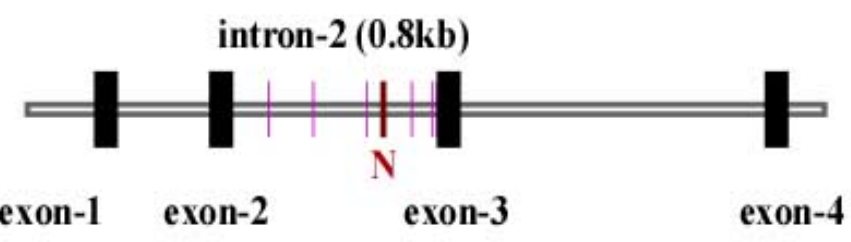

Fig. S21. Locations targeted by primers for mapping the TMPRSS2-ETV1 genomic breakpoints.

The locations of 23 forward primers (green) spacing across TMPRSS2 intron-1 ( 10 kb) and 7 reverse primers (purple) spacing across ETV1 intron-2 ( 0.8 kb). Each vertical line represents a target location by a primer or a set of nested primers. The specific primers that amplify the genomic breakpoint shown in Fig. 4E in the main text are labeled as red. 
bioRxiv preprint doi: https://doi.org/10.1101/151241; this version posted June 16, 2017. The copyright holder for this preprint (which was not certified by peer review) is the author/funder, who has granted bioRxiv a license to display the preprint in perpetuity. It is made available under aCC-BY 4.0 International license.
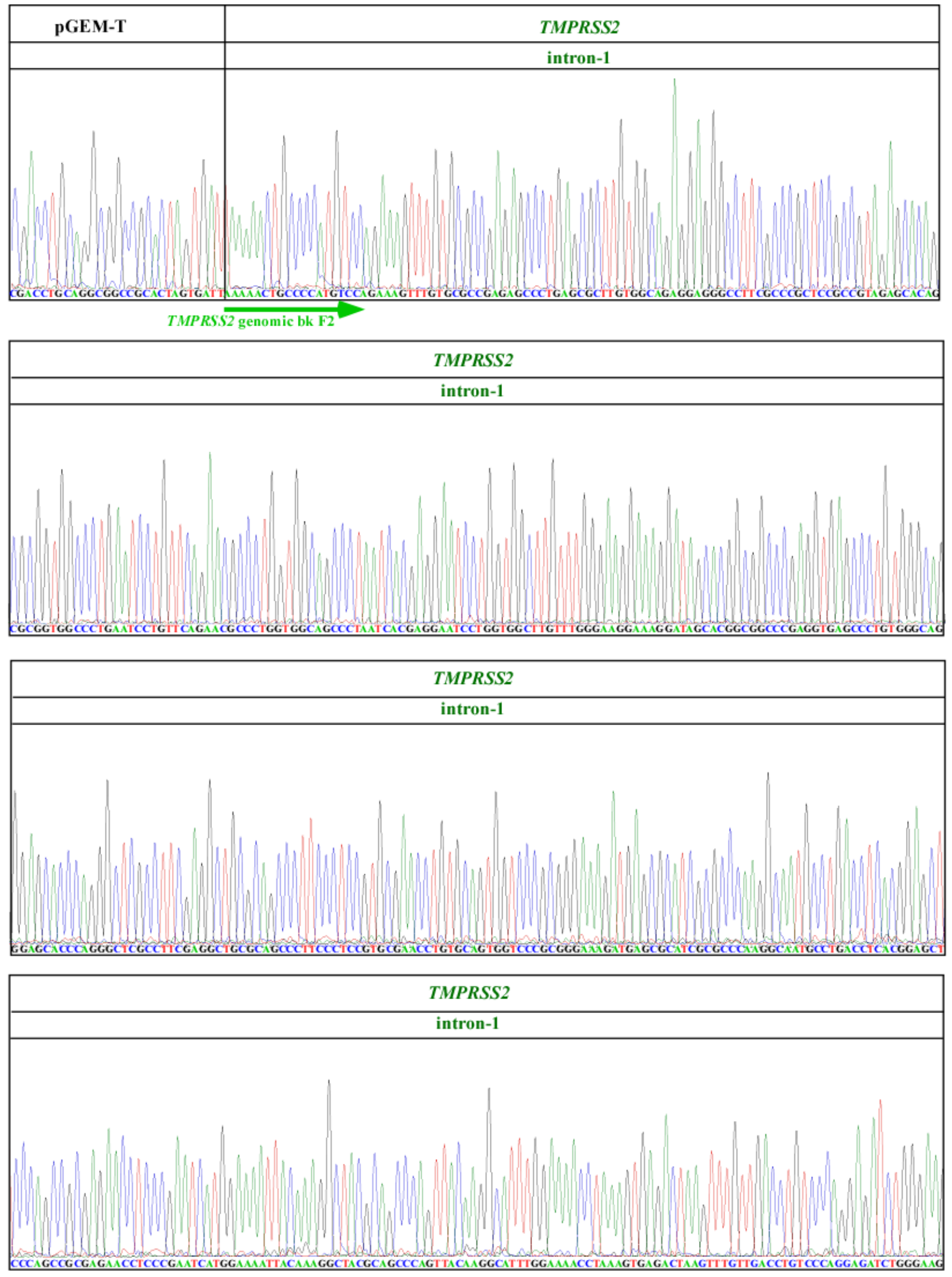
bioRxiv preprint doi: https://doi.org/10.1101/151241; this version posted June 16, 2017. The copyright holder for this preprint (which was not certified by peer review) is the author/funder, who has granted bioRxiv a license to display the preprint in perpetuity. It is made available under aCC-BY 4.0 International license.

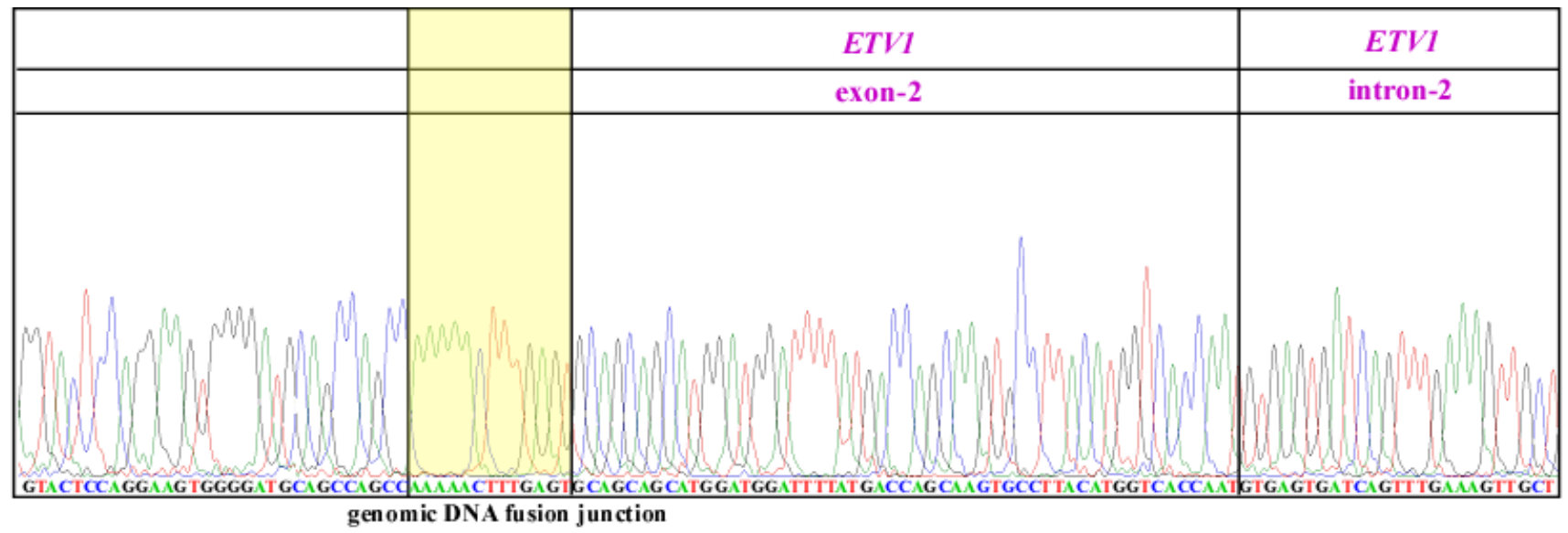

\section{ETV1}

intron-2
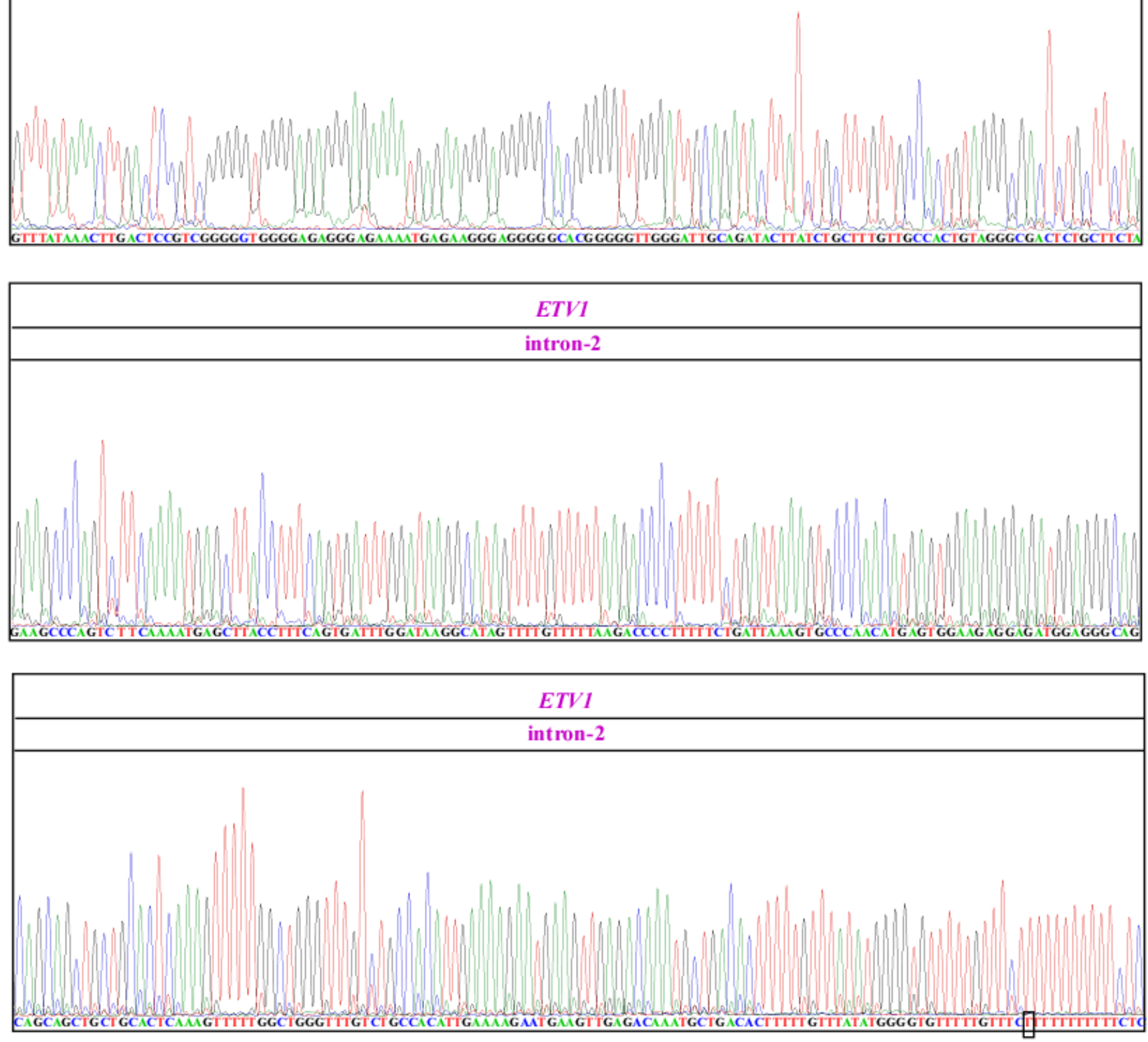

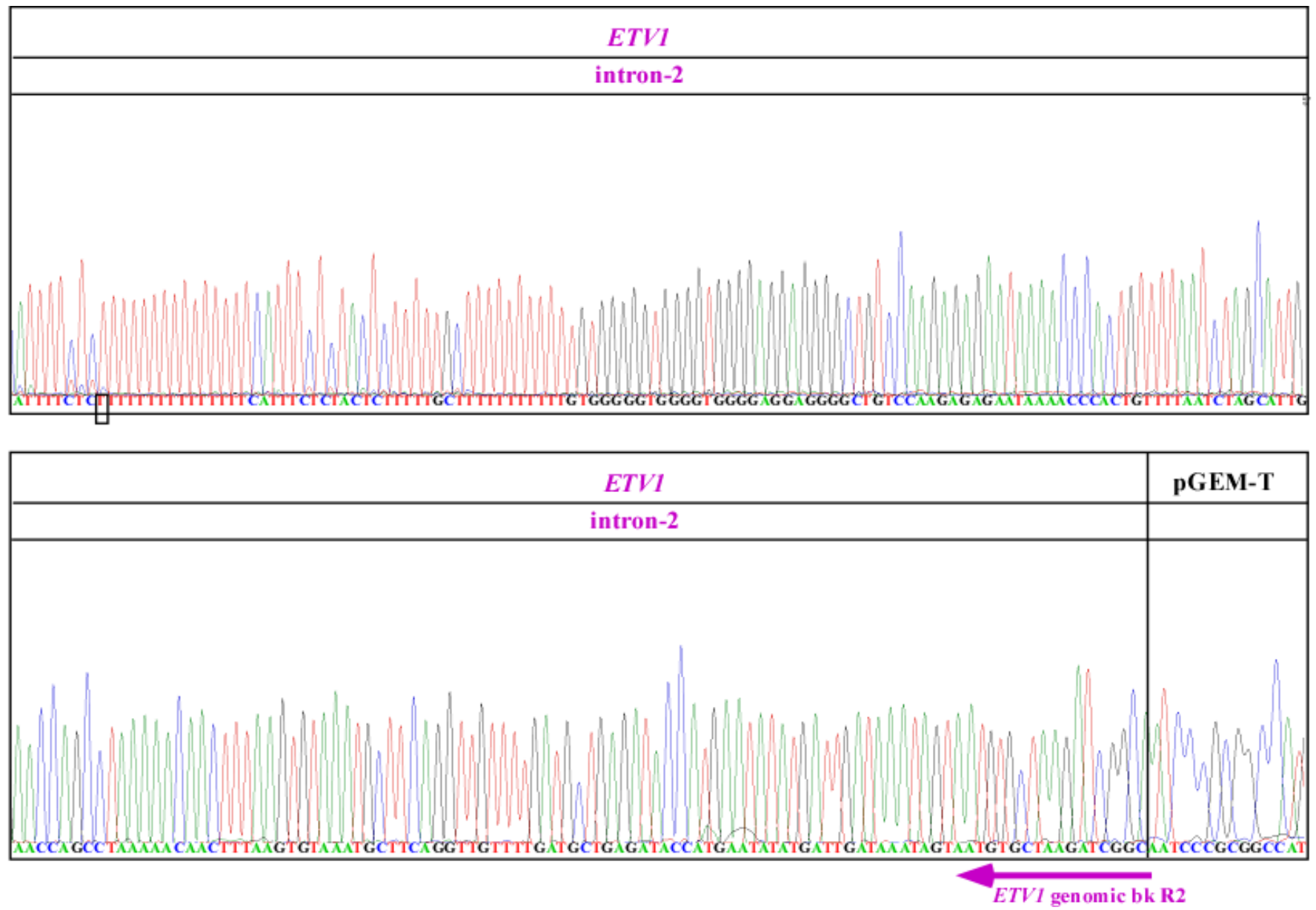

Fig. S22. Sanger sequencing confirmed that the induced TMPRSS2-ETV1 band $\mathrm{x}$ is due to gene fusion resulting from genomic arrangement.

LNCaP cells transfected with antisense-TMPRSS2-ETV1-A1 were enriched for the TMPRSS2ETV1 fusion transcript using a procedure similar to that described in Fig. S14. Genomic DNA PCR was performed on the enriched LNCaP population which yielded three gene fusion bands of 1150 bp (band x), 1044 bp (band y), 1043 bp (band z), as shown in Fig. 4E in the main text. Sanger sequencing chromatograms confirmed that the 1150 bp band $\mathrm{x}$ contains $\sim 485$ bp of TMPRSS2 intron-1 joined to 665 bp of ETV1 intron-3. The locations of the primers used to amplify the PCR product are shown as the green and purple arrows for TMPRSS2 intron-1 and ETV1 intron-3, respectively. Region of microhomology at the breakpoints are boxed by solid lines. The pGEM-T plasmid was used for cloning the genomic DNA PCR product. Mutations shown in black boxes are PCR artifacts because they are absent in other clones that were sequenced in parallel. 
bioRxiv preprint doi: https://doi.org/10.1101/151241; this version posted June 16, 2017. The copyright holder for this preprint (which was not certified by peer review) is the author/funder, who has granted bioRxiv a license to display the preprint in perpetuity. It is made available under

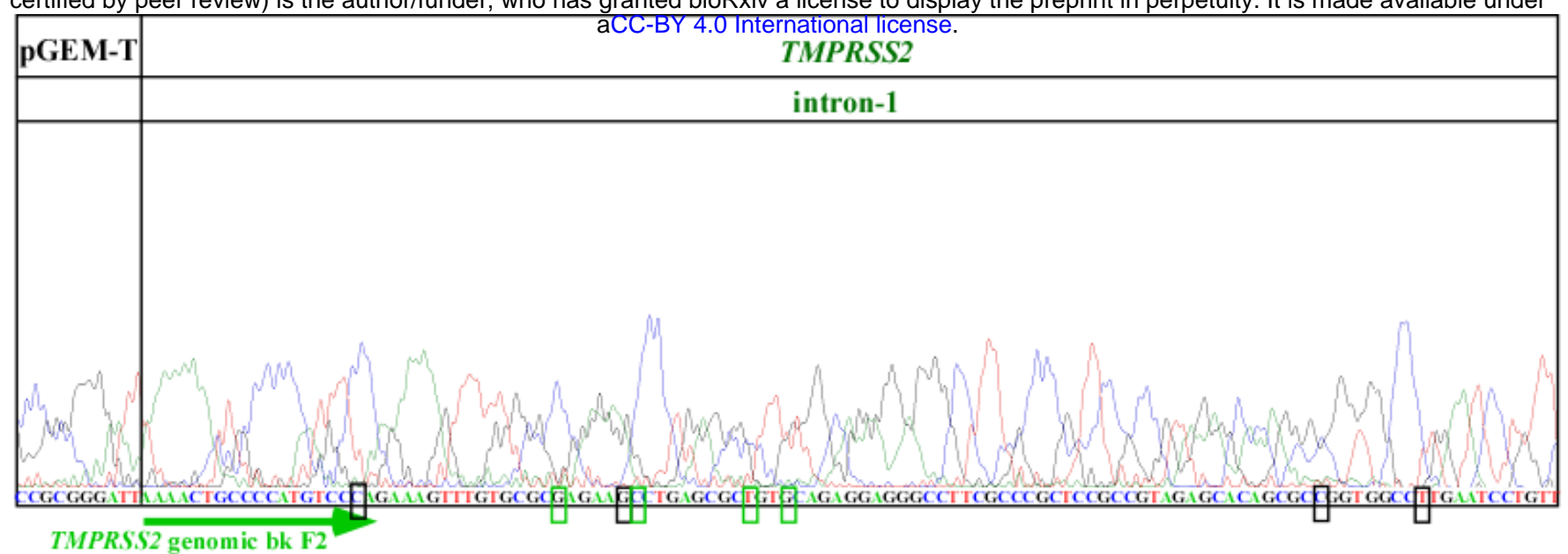

\section{TMPRSS2}

intron-1
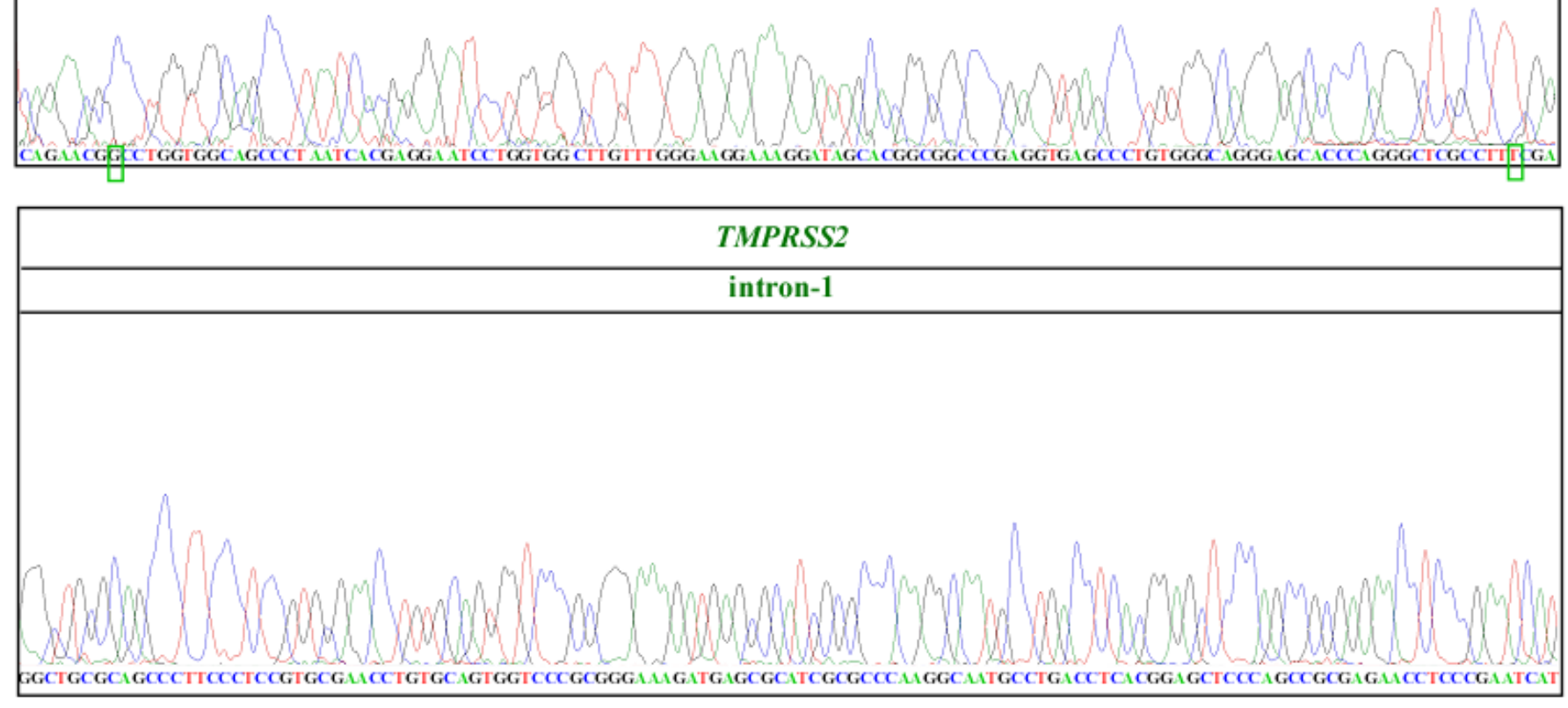

\section{TMPRSS2}

intron-1

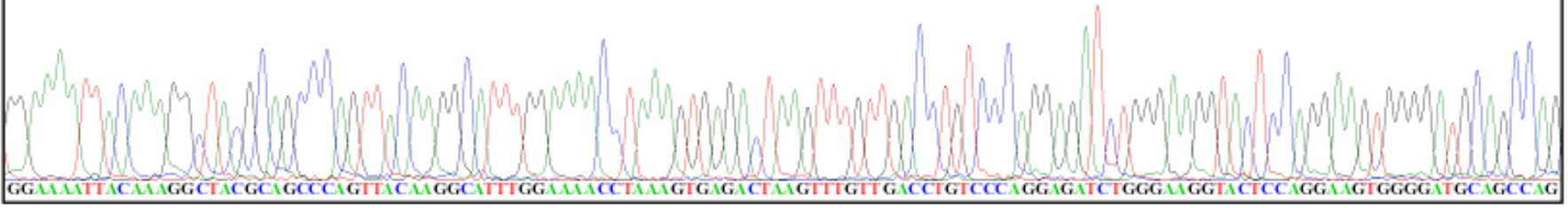


bioRxiv preprint doi: https://doi.org/10.1101/151241; this version posted June 16, 2017. The copyright holder for this preprint (which was not certified by peer review) is the author/funder, who has granted bioRxiv a license to display the preprint in perpetuity. It is made available under

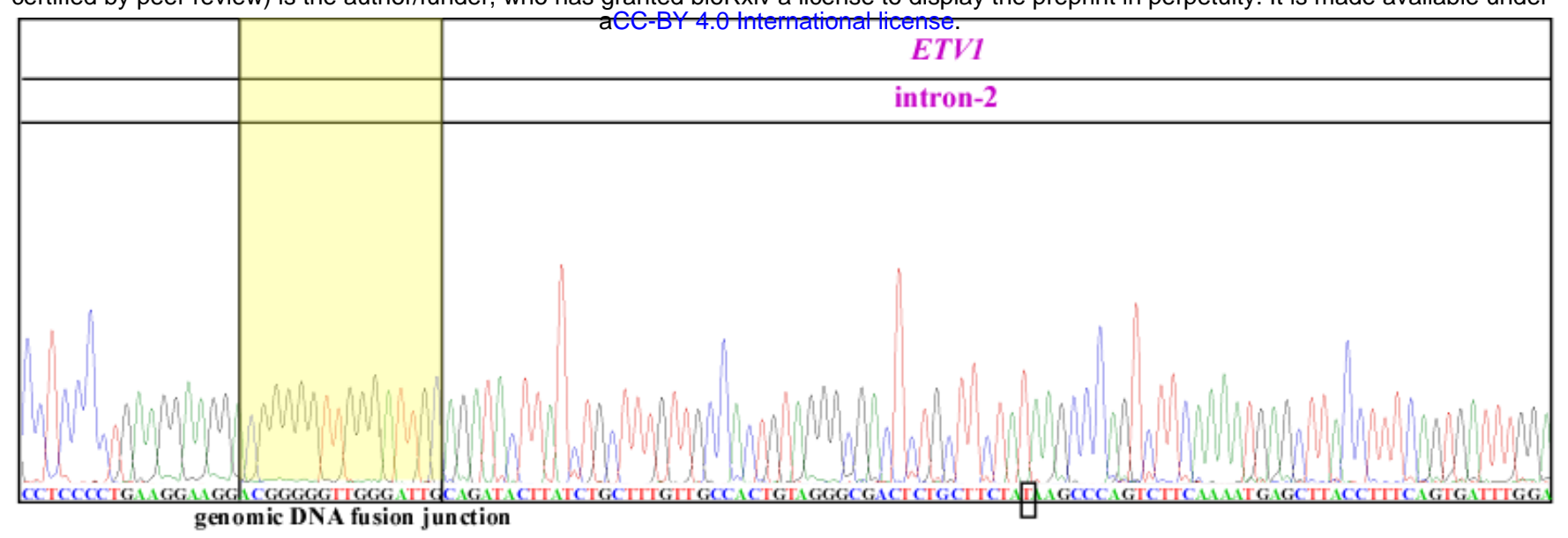

\section{ETV1}

intron-2
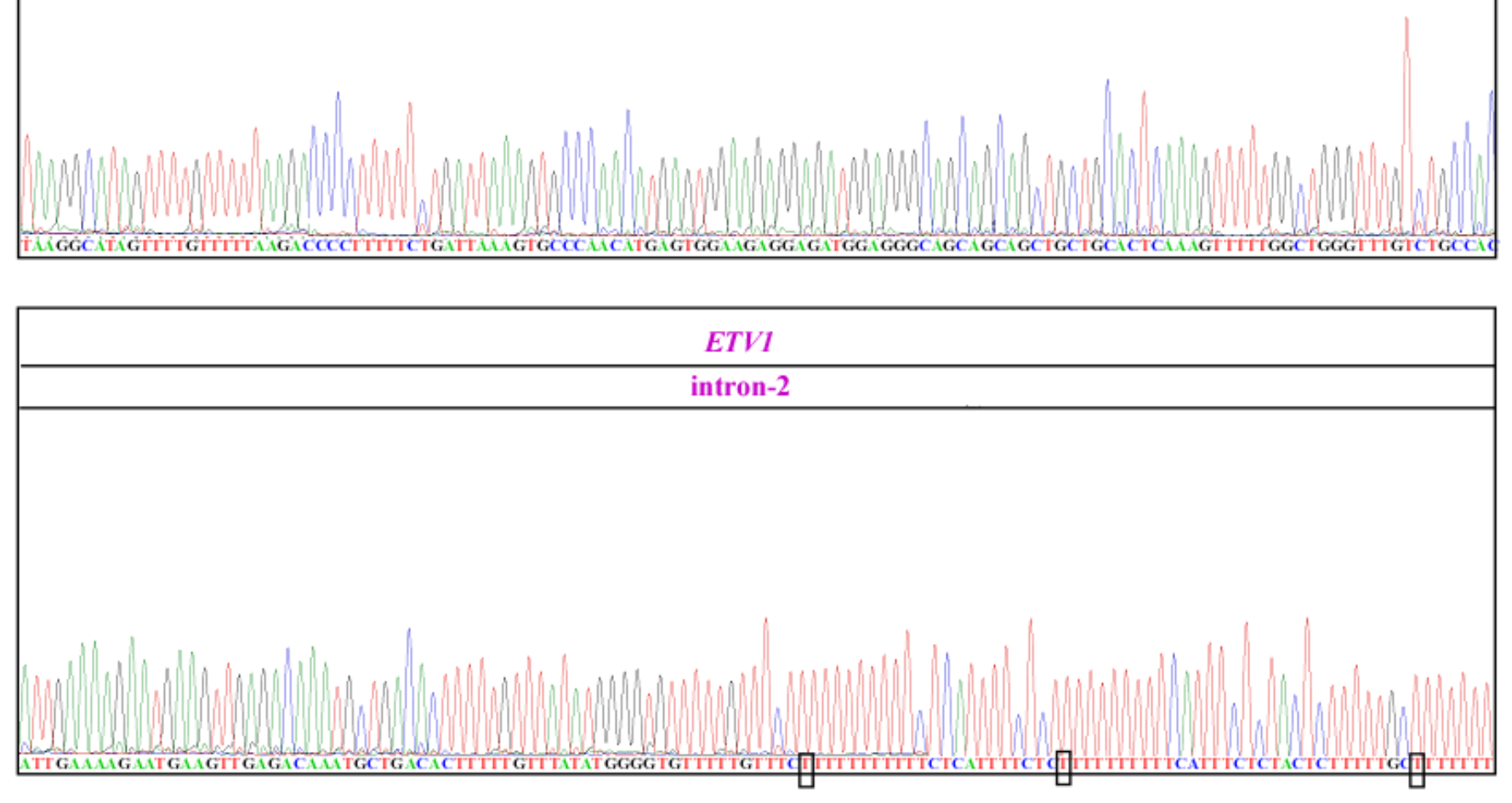

ETV1

intron-2

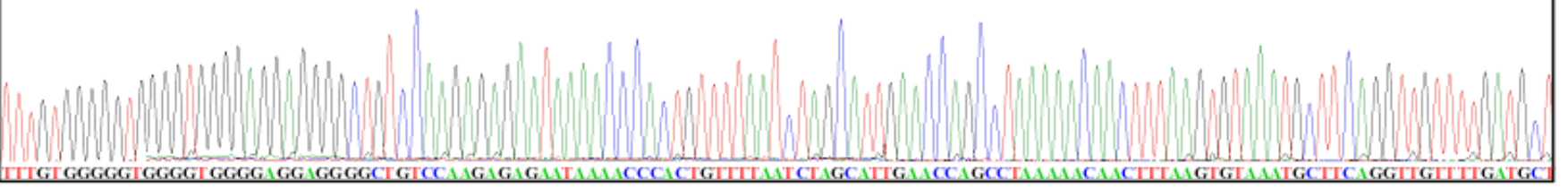




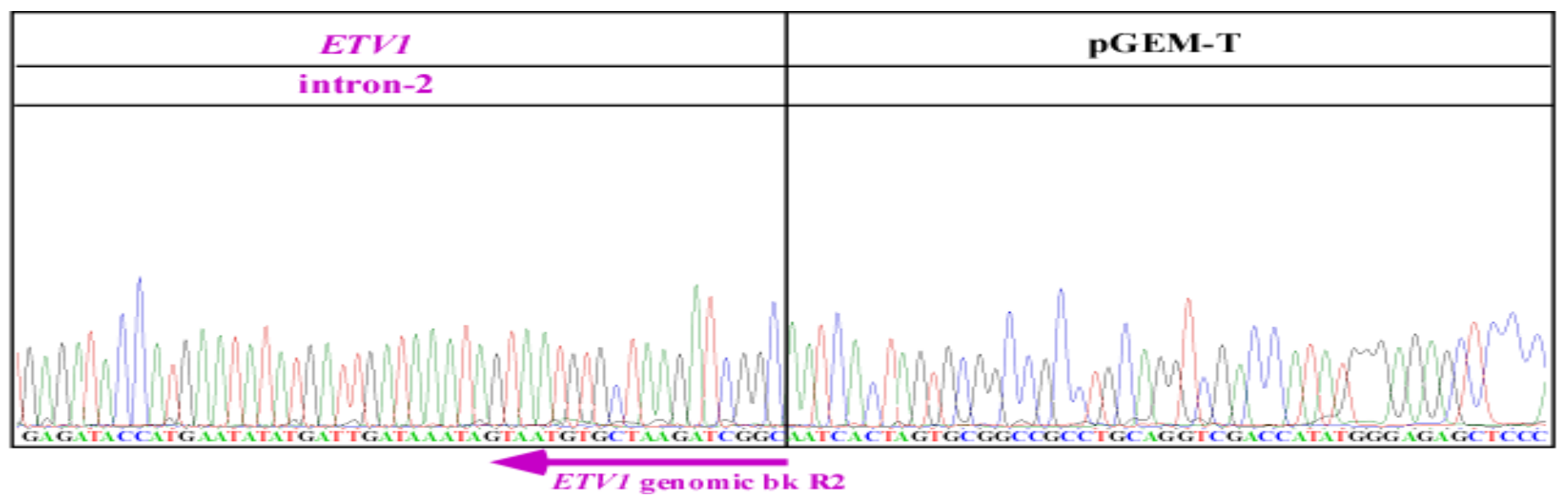

Fig. S23. Sanger sequencing confirmed that the induced TMPRSS2-ETV1 band $y$ is due to gene fusion resulting from genomic arrangement.

Similarly, Sanger sequencing was performed on the genomic PCR fusion bands 1044 bp (band y) shown in Fig. 4E in the main text. The resulting chromatograms confirmed that the $1044 \mathrm{bp}$ band y contains $~ 516$ bp of TMPRSS2 intron- 1 joined to $\sim 528$ bp of ETV1 intron-3. The locations of the primers used to amplify the PCR product are shown as the green and purple arrows for TMPRSS2 intron-1 and ETV1 intron-3, respectively. Region of microhomology and indels at the breakpoints are boxed by solid lines. The pGEM-T plasmid was used for cloning the genomic DNA PCR product. Mutations shown in small black boxes are PCR artifacts because they are absent in other clones that were sequenced in parallel, whereas mutations shown in small green boxes are either PCR introduced or not verified because of poor sequencing quality in other clones that were sequenced in parallel. 
bioRxiv preprint doi: https://doi.org/10.1101/151241; this version posted June 16, 2017. The copyright holder for this preprint (which was not certified by peer review) is the author/funder, who has granted bioRxiv a license to display the preprint in perpetuity. It is made available under

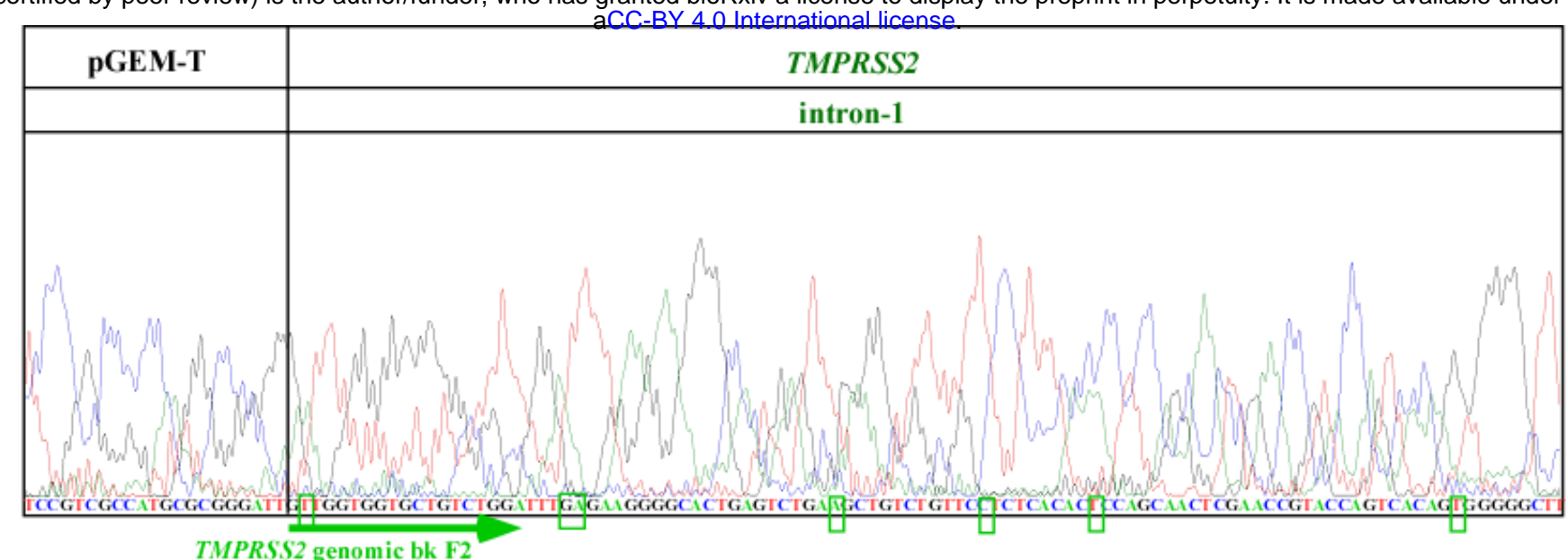
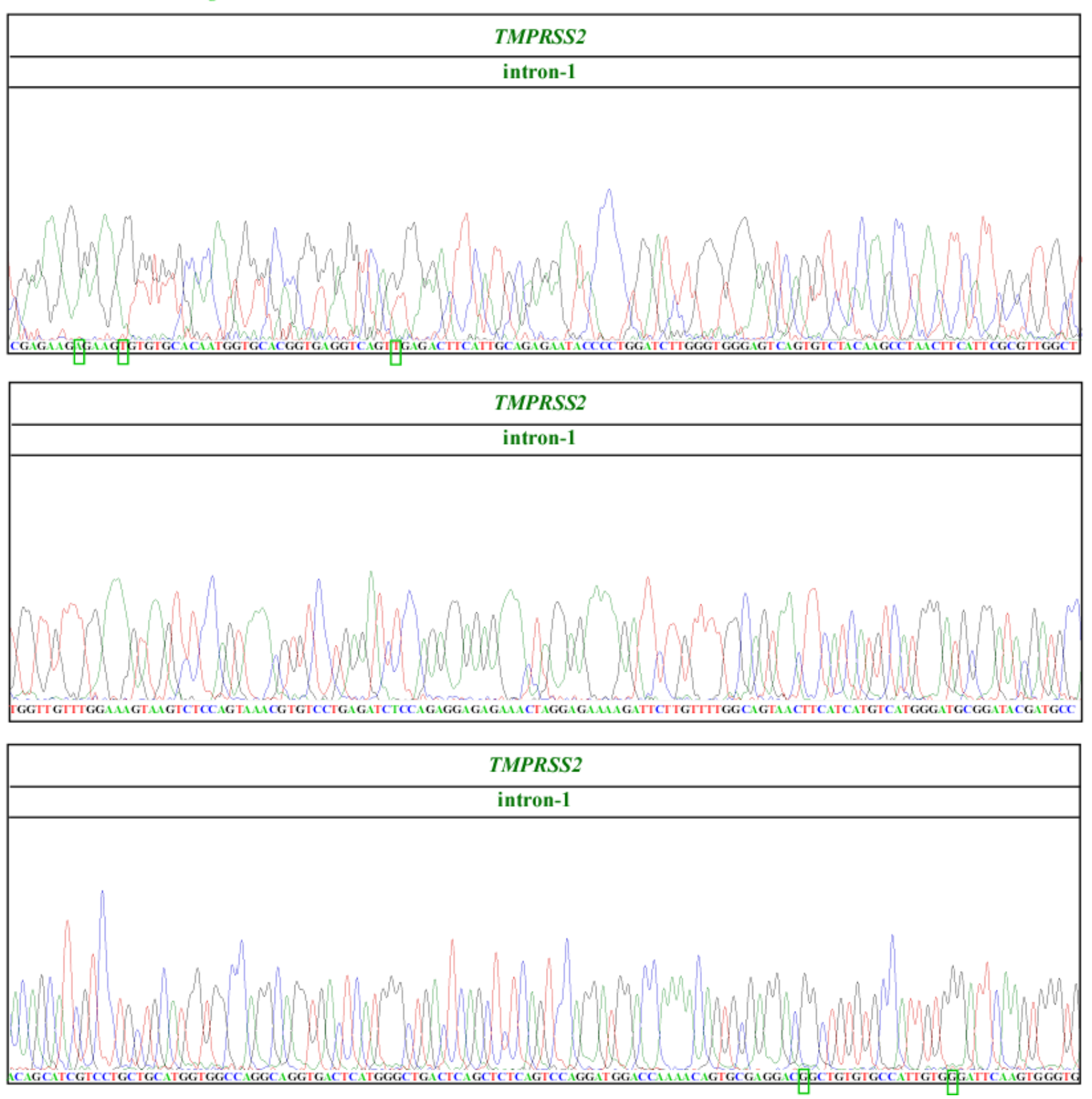
bioRxiv preprint doi: https://doi.org/10.1101/151241; this version posted June 16, 2017. The copyright holder for this preprint (which was not certified by peer review) is the author/funder, who has granted bioRxiv a license to display the preprint in perpetuity. It is made available under

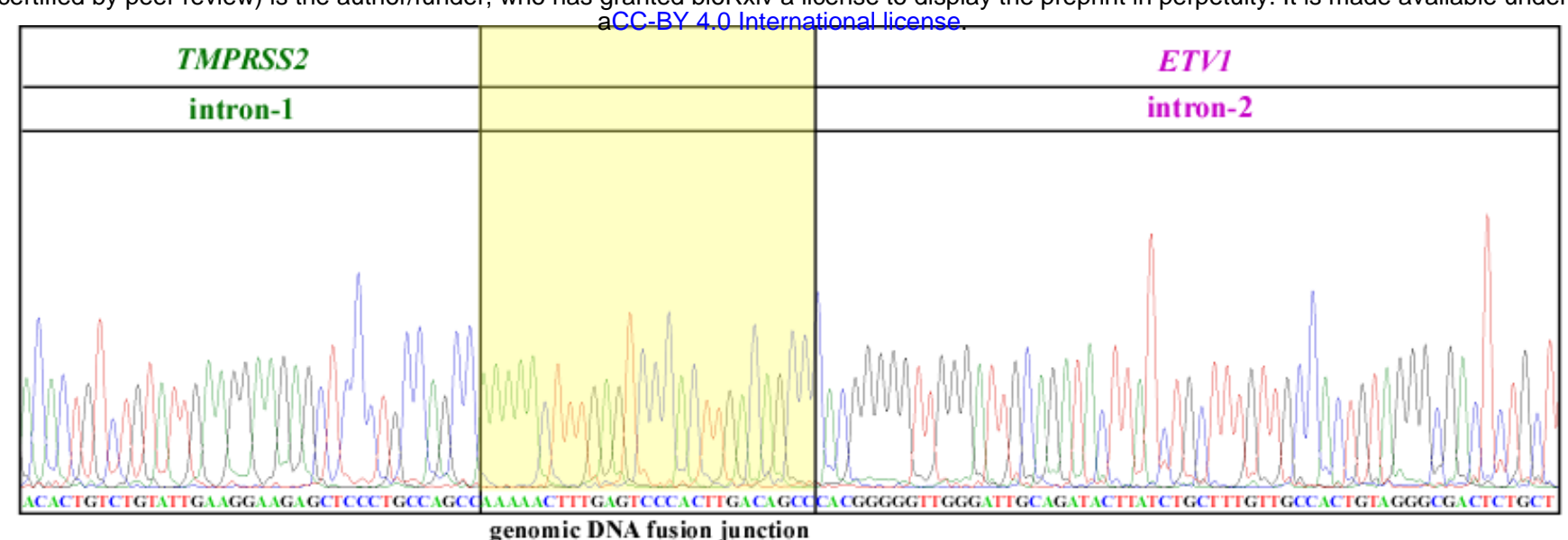

\section{ETV1}

intron-2
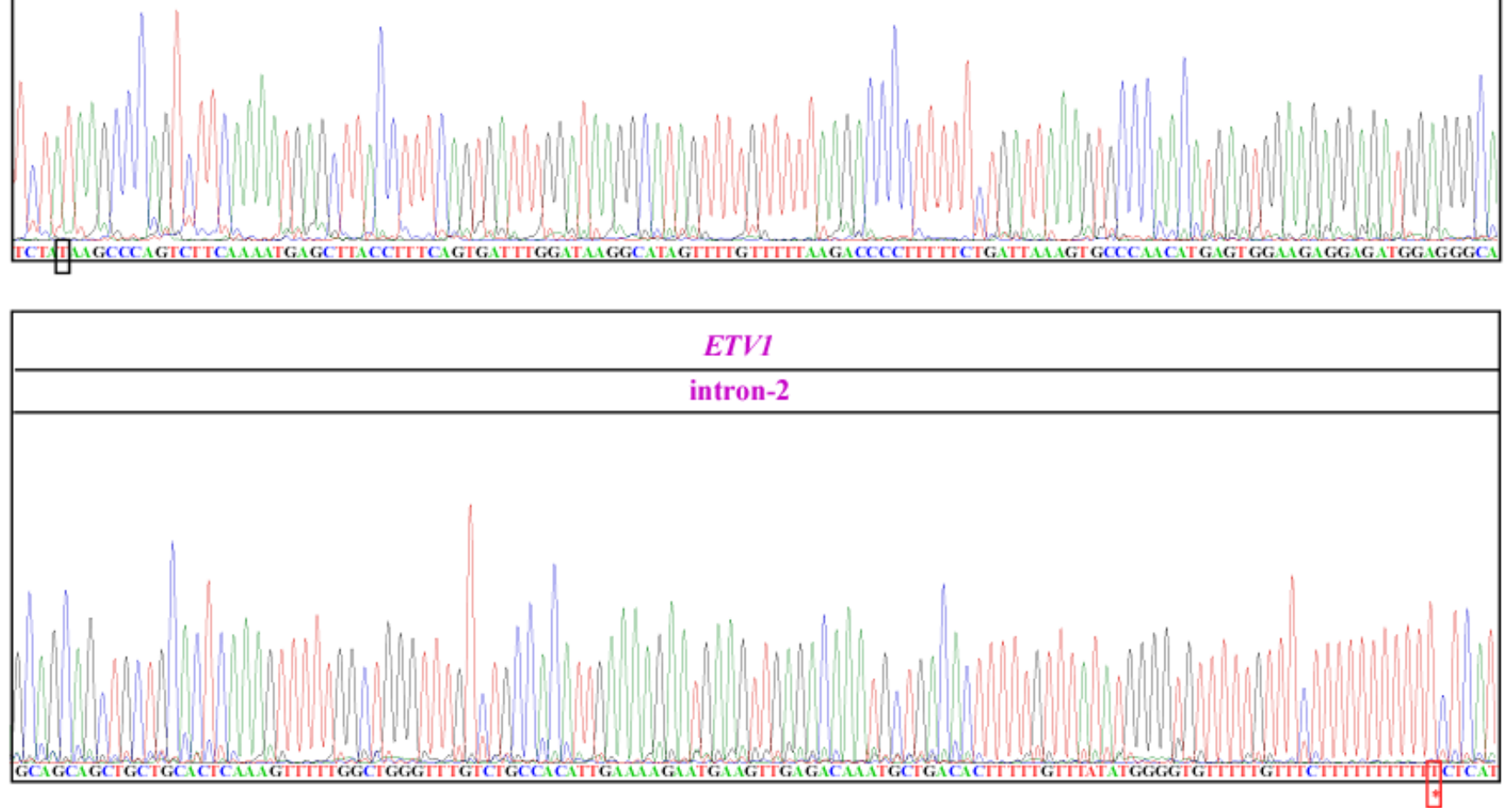

ETV1

intron-2

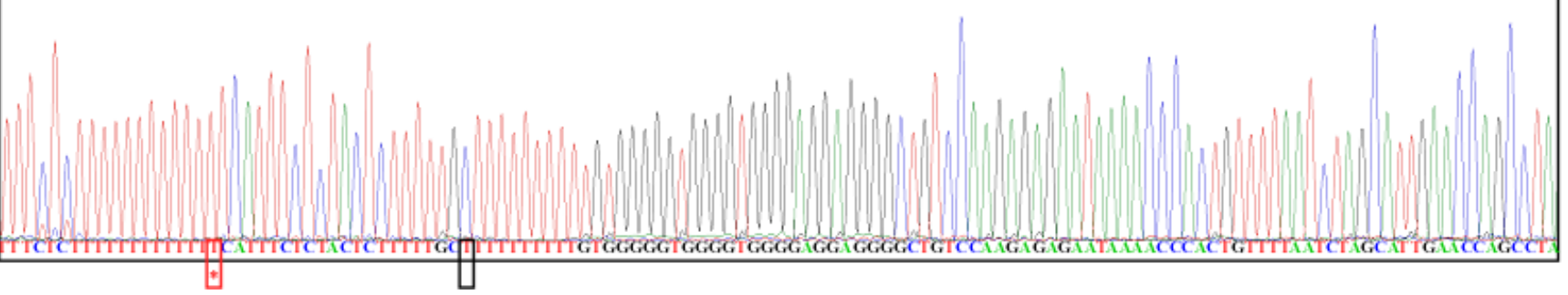




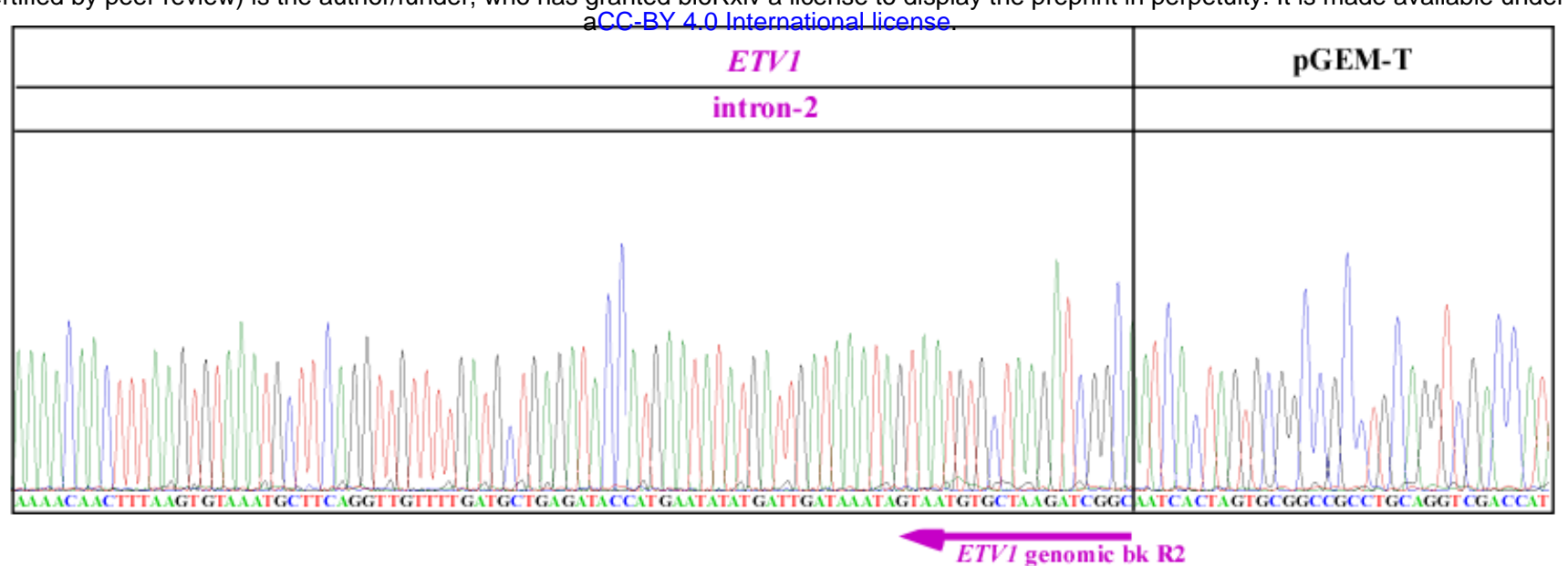

Fig. S24. Sanger sequencing confirmed that the induced TMPRSS2-ETV1 band $\mathrm{z}$ is due to gene fusion resulting from genomic arrangement.

Similarly, Sanger sequencing was performed on the genomic PCR fusion bands 1043 bp (band z) shown in Fig. 4E in the main text. The resulting chromatograms confirmed that the $1043 \mathrm{bp}$ band z contains $\sim 514$ bp of TMPRSS2 intron-1 joined to 529 bp of ETV1 intron-3. The locations of the primers used to amplify the PCR product are shown as the green and purple arrows for TMPRSS2 intron-1 and ETV1 intron-3, respectively. Region of microhomology and indels at the breakpoints are boxed by solid lines. The pGEM-T plasmid was used for cloning the genomic DNA PCR product. Mutations shown in small black boxes are PCR artifacts because they are absent in other clones that were sequenced in parallel, whereas mutations shown in small green boxes are either PCR introduced or not verified because of poor sequencing quality in other clones that were sequenced in parallel. 


\section{Movies S25a \& b. Examples of TMPRSS2-ETV1 fusion gene identified by 3D-microscopy FISH image reconstruction.}

The enriched TMPRSS2-ETV1 LNCaP cell population was fixed with formaldehyde followed by hybridization with FISH probes against TMPRSS2 gene (red) and ETV1 gene (green). Images were acquired on a GE Healthcare DeltaVision image restoration microscope with an Olympus UPLS Apo 100x/1.4 NA and 2k x 2k EDGE/sCMOS camera. Z stacks were acquired with spacing of $0.25 \mathrm{um}$. Images were deconvolved using conservative algorithm in Resolve3D SoftWorx (version 6.5.2). Max projection and volume visualization was also performed with the same software. S25a represents 3-D along x-axis and S25b along y-axis. 


\section{Materials and Methods}

\section{LNCaP cell culture}

LNCaP cells were routinely cultured in RPMI 1640 medium (RPM1 1640, 1X, with Lglutamine, \#10-040-CV, CORNING cellgro) containing 10\% fetal bovine serum (premium grade FBS, \#1500-500, Seradigm) and 1\% penicillin/streptomycin (\#15140-122, Gibco) in a $5 \% \mathrm{CO}_{2}$ humidified incubator. For experiments involving the induction of fusion gene by input RNA, regular fetal bovine serum in the culture medium was replaced by Charcoal:Dextran stripped fetal bovine serum (catalog\#100-119, Gemini Bioproducts) to remove hormones present in serum. LNCaP cells were cultured in this special medium for 24 hrs prior to plasmid transfection.

\section{PNT1A cell culture}

PNT1A cells were routinely cultured in RPMI 1640 medium containing 10\% fetal bovine serum (premium grade FBS, \#1500-500, Seradigm) and 1\% penicillin/streptomycin (\#15140122 , Gibco) in a $5 \% \mathrm{CO}_{2}$ humidified incubator.

\section{Transient transfection of plasmids for expressing the input RNAs}

Twenty hours prior to transfection, LNCaP cells were seeded in 12-wells plate (BioLite 12 Well Multidish, \#130185, Thermo Fisher Scientific) with a density of $5 \times 10^{5}$ cells/well and 1 $\mathrm{ml} /$ well of culture medium containing Charcoal:Dextran stripped fetal bovine as described above. Transfection was performed using Turbofect transfection reagent (Thermo Scientific, \#R0531) according to manufacturer's protocol. Briefly, $1 \mu \mathrm{g}$ of a particular plasmid was first diluted in $100 \mu \mathrm{l}$ of the serum-free DMEM followed by immediate mixing by pipetting. $4 \mu \mathrm{l}$ of the transfection reagent was then added to the diluted DNA followed by mixing and incubation for $20 \mathrm{~min}$. The DNA/transfection reagent mixture was then added drop wise to a well containing LNCaP cells in $1 \mathrm{ml}$ medium.

For transfection in PNT1A cells, $5 \times 10^{5}$ cells/well were plated in 12-wells plate in $1 \mathrm{ml} /$ well of cultured medium 24 hrs prior to transfection. Transfection was performed using the same formula described for LNCaP cells. For repetitive transfections, initially transfected PNT1A cell population were split every three days, half was processed for RT-PCR assay and half was seeded again in a new well for the next transfection.

\section{DHT preparation and treatment}

DHT (Dihydrotestosterone) was purchased from Sigma Adlrich (5 $\alpha$-Androstan-17 $\beta$-ol-3-one, \#A8380). Concentrated stock of $1500 \mu \mathrm{M}$ was prepared by dissolving $4.3566 \mathrm{mg}$ of DHT powder in $10 \mathrm{ml}$ of 100\% ethanol (200 proof ethanol, Koptec, \#V1016) and then aliquoted in $1 \mathrm{ml}$ tubes and stored at $-80^{\circ} \mathrm{C}$. 
For treating cultured cells, concentrated DHT stock was diluted as 10x working solutions (for example, for $0.9 \mu \mathrm{M}$ final concentration, $10 \mathrm{x}$ is prepared as $9.0 \mu \mathrm{M}$ ) with the appropriate complete culture medium and used immediately. Complete media for LNCaP cells: RPMI $1640+10 \%$ Charcoal:Dextran stripped fetal bovine serum $+1 \%$ penicillin/streptomycin. Complete media for PNT1A: RPMI $1640+10 \%$ fetal bovine serum $+1 \%$ penicillin/streptomycin. Six hrs post transfection, $111 \mu$ l of fresh 10x DHT working solutions was added to each well of 12-wells plate containing $1 \mathrm{ml}$ medium and transfected cells.

For long-term treatment, medium was changed with fresh DHT every three days.

\section{RNA isolation}

Total RNA from cultured cells was extracted using High Pure RNA isolation Kit according to manufacturer's instructions (\#11828665001, Roche). Briefly, cells were suspended in $200 \mu$ l of PBS buffer and were then lysed with $400 \mu$ l of lysis buffer. The sample was then passed through the filter assembly resulting in the binding of the nucleic acids to the filter. The filter containing nucleic acids was then incubated with DNase I dissolved in DNase incubation buffer to degrade genomic and plasmid DNAs. The column was then rinsed with wash buffer and total RNA then eluted in a new tube for further analysis.

For detection of residual genomic and plasmid DNA, eluted RNA was subject to PCR reaction with primers specific to intron regions of house-keeping gene GAPDH, and with primers specific to plasmid transfected. Total RNA was converted to cDNA only if it is validated as free of DNA contamination.

\section{Reverse transcription reaction}

$1 \mu \mathrm{g}$ of total RNA was used for each reverse transcription reaction according to manufacturer instruction (superscript III RT, \# 18080-051, Invitrogen). RNA was converted to cDNA either with Oligo dT primer (for induced fusion transcripts) or with random hexamers (for input RNAs expressed by U6 promoter). After the addition of dNTPs, the mixture was denatured at $65^{\circ} \mathrm{C}$ for 5 minutes. This was followed by the addition of a master-mix containing $1 \times$ superscript buffer, $10 \mathrm{mM}$ DTT, $5 \mathrm{mM}$ Magnesium chloride, RNaseOUT and Superscript III reverse transcriptase. Reactions were carried out at $50^{\circ} \mathrm{C}$ for 50 minutes and then terminated by incubation at $85^{\circ} \mathrm{C}$ for 5 minutes. cDNA was then treated with RNase-H for 20 minutes at $37^{\circ} \mathrm{C}$ to degrade RNA in DNA/RNA hybrid. $1 \mu \mathrm{l}$ of cDNA was used as template for each subsequent PCR reaction.

\section{RT-PCR for detecting induced fusion transcripts}

The majority of induced fusion RNAs in this manuscript were detected using one-round RTPCR. The following cases were assayed using three-round nested PCR: (1) the results of DHT treatment at physiological concentrations as shown in Fig. 1D and 6A, (2) the induction of TMPRSS2-ERG fusion transcript in non-malignant PNT1A cells as shown in Fig. 3F, (3) 
the specificity of input RNAs assayed in Fig. 4C, and (4) endogenous ERG level detection in LNCaP cells in Fig. S13. The following cases were assayed using two-round nested PCR: the induction of TMPRSS2-ETV1 fusion RNA as shown in Fig. 4A and 4B, and the detection of TMPRSS2-ETV1 fusion in the enriched population in Fig. 4D.

PCR was done with a standard three-step protocol using REDTaq DNA polymerase (\#D5684-1KU, Sigma) according to manufacturer instruction.

Reaction was set as follows:

\section{PCR reaction:}

Forward primer :

Reverse primer :

10x reaction buffer:

dNTPs:

DMSO:

cDNA:

Autoclaved Milli-Q water:

REDTaq DNA polymerase:
$1.0 \mu \mathrm{l}$ (from $10 \mu \mathrm{M}$ stock, Sigma)

$1.0 \mu \mathrm{l}$ (from $10 \mu \mathrm{M}$ stock, Sigma)

5.0 $\mu$ l (comes with REDTaq, Sigma)

1.0 $\mathrm{pl}$ (from 10mM stock, \#11969064001, Roche)

1.5 pl (\#154938, Sigma-Aldrich)

$1.0 \mu$ l (from $20 \mu \mathrm{l}$ stock prepared from $1 \mu \mathrm{g}$ RNA)

$38.5 \mu l$

1.0 $\mu \mathrm{l}$ (\#D5684-1KU, Sigma)

Total volume:

$50 \mu \mathrm{l}$

Standard one-round PCR conditions for TMPRSS2-ERG:

Pre-denaturation $\quad 94^{0} \mathrm{C}, 4 \mathrm{~min}$

Denaturation $\quad 94^{0} \mathrm{C}, 30 \mathrm{sec}$

32 cycles for induced fusion RNA

Annealing

$58^{0} \mathrm{C}, 45 \mathrm{sec}$

27 cycles for input RNA

Extension

$72^{\circ} \mathrm{C}, 60 \mathrm{sec}$

Final Extension $\quad 72^{0} \mathrm{C}, 5 \mathrm{~min}$

PCR conditions for three-round nested PCR for TMPRSS2-ERG:

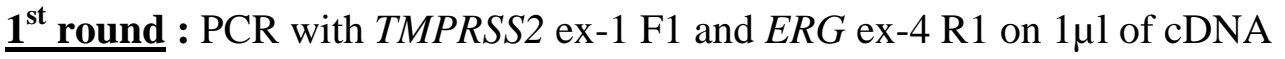

Pre-denaturation $94^{0} \mathrm{C}, 4 \mathrm{~min}$

Denaturation $\quad 94^{0} \mathrm{C}, 30 \mathrm{sec}$

Annealing $\quad 58^{0} \mathrm{C}, 45 \mathrm{sec}$

32 cycles for induced fusion RNA

Extension

$72^{0} \mathrm{C}, 60$ sec

Final Extension $\quad 72^{0} \mathrm{C}, 5 \mathrm{~min}$ 
$\underline{2^{\text {nd }}}$ round : PCR with TMPRSS2 ex-1 F2 and ERG ex-4 R2 on $1 \mu \mathrm{l}$ of $1^{\text {st }}$ round product, PCR conditions same as $1^{\text {st }}$ round.

$\underline{3}^{\text {rd }}$ round : PCR with TMPRSS2 ex-1 F3 and ERG ex-4 R3 on $1 \mu$ l of $2^{\text {nd }}$ round product, PCR conditions same as $1^{\text {st }}$ round.

PCR conditions for two-round nested PCR for TMPRSS2-ETV1:

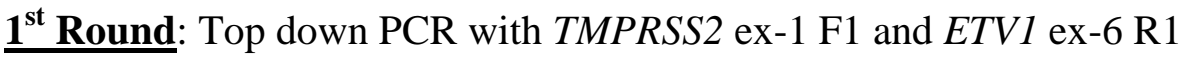

$\left.\begin{array}{lc}\text { Denaturation } & 94^{0} \mathrm{C}, 30 \mathrm{sec} \\ \text { Annealing } & * \quad, 45 \mathrm{sec} \\ \text { Extension } & 72^{0} \mathrm{C}, 60 \mathrm{sec}\end{array}\right]$ cycles

$* 2$ cycles at each temperature: $62^{0} \mathrm{C}, 61^{0} \mathrm{C}, 60^{\circ} \mathrm{C} \ldots \ldots \ldots \ldots . . .49^{0} \mathrm{C}$ followed by

$\left.\begin{array}{ll}\text { Denaturation } & 94^{0} \mathrm{C}, 30 \mathrm{sec} \\ \text { Annealing } & 48{ }^{\circ} \mathrm{C}, 45 \mathrm{sec} \\ \text { Extension } & 72^{\circ} \mathrm{C}, 60 \mathrm{sec}\end{array}\right]$ cycles

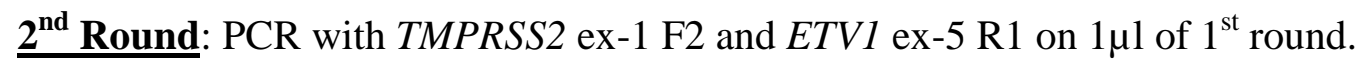

Pre-denaturation $94^{0} \mathrm{C}, 4 \mathrm{~min}$

Denaturation $\quad 94^{0} \mathrm{C}, 30 \mathrm{sec}$

Annealing $\quad 57^{0} \mathrm{C}, 45 \mathrm{sec} \quad 32$ cycles for Induced fusion RNA

Extension $\quad 72^{0} \mathrm{C}, 60 \mathrm{sec}$

Final Extension $\quad 72^{0} \mathrm{C}, 5 \mathrm{~min}$

$\underline{3}^{\text {rd }}$ round (for Fig. 4D): PCR with TMPRSS2 ex-1 F3 and ETV1 ex-5 R2 on $1 \mu$ l of $2^{\text {nd }}$ round, PCR conditions same as $2^{\text {nd }}$ round. 


\section{Long range PCR for detecting genomic DNA fusion junction}

Nested long-range PCRs according to the manufacturer's protocols using LA PCR kit (Takara, \# RR002M). 200 ng of genomic DNA was used in each reaction and PCR was performed with annealing and extension at $68^{\circ} \mathrm{C}$ for 20 minutes. $1 \mu l$ from the above reaction (1st round PCR) was used as template for the 2nd round PCR.

For the genomic breakpoint identified in this manuscript, 1st round long range PCR was done using primers TMPRSS2 genomic bk-F1 and ERG genomic bk-R1 shown in primer list below. $1 \mu$ l from the above reaction (1st round PCR) was used as template for the 2nd round PCR using inner primers TMPRSS2 genomic bk-F2 and ERG genomic bk-R2.

\section{Cloning and Sanger sequencing of induced fusion transcripts}

PCR amplified cDNA bands were excised from the gel and eluted using QIAquick Gel Extraction Kit (\#28706, Qiagen). The eluted bands were then cloned to pGEM-T vector (pGEM-T vector system I, \# A3600) following manufacturer instruction. Sanger sequencing was performed using the service of Beckman Coulter Genomics.

\section{Tm calculations}

Melting temperature (Tm) of putative genomic DNA stems were calculated using the following formula (Rychlik and Rhoads, 1989):

$$
\operatorname{Tm}\left({ }^{\circ} \mathrm{C}\right)=4 \times \text { (number of G's and C's) }+2 \times \text { (number of A's and T's) }
$$

A high energy $\mathrm{G} \cdot \mathrm{T}$ and $\mathrm{A} \cdot \mathrm{C}$ wobble pair known to have Watson-Crick like geometry in DNA double helix (Kimsey and Al-Hashimi, 2014; Watson and Crick, 1953) are considered as having the same stability as an A·T pair.

\section{Fluorescent in situ hybridization (FISH)}

Enriched population carrying TMPRRS2-ETV1 fusion events were first grown on $18 \mathrm{~mm}$ round \#1 coverglass in a 12-well cell culture plate at the initial density of $200-400 \mathrm{k} / \mathrm{well}$. Cells were then fixed with $4 \%$ (vol./vol.) formaldehyde followed by denaturation of DNA with $0.1 \mathrm{~N} \mathrm{HCl}$ for $5 \mathrm{~min}$ and with $70 \%$ formamide at $85^{\circ} \mathrm{C}$ for $7 \mathrm{~min}$. Hybridization of target DNA with probes were done at $37^{\circ} \mathrm{C}$ for $16 \mathrm{hr}$ in a humidified chamber. Cells were then washed, stained with DAPI and imaged with microscope. FISH probes for TMPRSS2 (RP1135C4, red) and ETV1 (RP11-769K2, green) were purchased from Empire Genomics. 


\section{$\alpha$-Amanitin assay}

Twenty hours prior to transfection, LNCaP cells were seeded in 12-wells plate (BioLite 12 Well Multidish, \#130185, Thermo Fisher Scientific) with a density of 5x10 $\mathrm{cells} /$ well and transfection was performed using Turbofect transfection reagent (Thermo Scientific, \#R0531) as described earlier. DHT was added at the final concentration of $0.9 \mu \mathrm{M}$ six hours post transfection. Following overnight incubation, cells were then treated with $4 \mu \mathrm{g} / \mathrm{ml} \alpha$ amanitin for various time periods (0, 2, 6, 12 and 24 hours). Cells were then revived in fresh medium containing $0.9 \mu \mathrm{M}$ DHT without $\alpha$-amanitin and RT-PCR was performed for either TMPRSS2-ERG or TMPRSS2-ETV1 fusion.

\section{Input RNA sequences}

\section{Sense-1}

+1tcagatcgcctggagacgccatccacgctgttttgacctccatagaagacaccgggacc gatccagcctccctcgaagctgatcctgagaacttcaggctcctgggcaacgtgctggtc tgtgtgctggcccatcactttggcaaagaattcGAGTAGGCGCGAGCTAAGCAGGAGGCGG AGGCGGAGGCGGAGGGCGAGGGGCGGGGAGCGCCGCCTGGAGCGCGGCAGGAAGCCTTATC AGTTGTGAGTGAGGACCAGTCGTTGTTTGAGTGTGCCTACGGAACGCCACACCTGGCTAAG ACAGAGATGACCGCGTCCTCCTCCAGCGACTATGGACAGACTTCCAAGATGAGCCCACGCG TCCCTCAGCAGGATTGGCTGTCTCAACCCCCAGCCAGGGTCACCATCAAAATGGAATGTAA CCCTAGCCAGGTGAATGGCTCAAGaagct tatcgataccgtcgacctcgagggcccagatc taattcaccccaccagtgcaggctgcctatcagaaagtggtggctggtgtggctaatgccc tggcccacaagtatcactaagctcgctttcttgctgtccaatttctattaaaggttccttt gttccctaagtccaactactaaactgggggatattatgaagggccttccggagcatctgga ttctgcctaataaaaaacatttatttcattgcaaaaaa........a

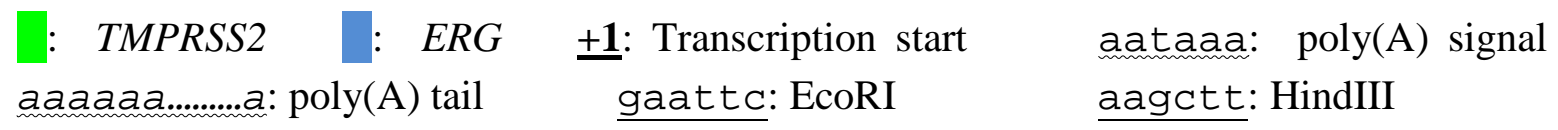

\section{Sense-2}

+1tgtgctcgcttcggcagcacatatactaacattggaacgatcctgcagGAGTAGGCGCG AGCTAAGCAGGAGGCGGAGGCGGAGGCGGAGGGCGAGGGGCGGGGAGCGCCGCCTGGAGCG CGGCAGGAAGCCTTATCAGTTGTGAGTGAGGACCAGTCGTTGTTTGAGTGTGCCTACGGAA CGCCACACCTGGCTAAGACAGAGATGACCGCGTCCTCCTCCAGCGACTATGGACAGACTTC CAAGATGAGCCCACGCGTCCCTCAGCAGGATTGGCTGTCTCAACCCCCAGCCAGGGTCACC ATCAAAATGGAATGTAACCCTAGCCAGGTGAATGGCTCAAG 
TMPRSS2 : ERG 푸: Transcription start $\underline{\text { ctgcag: }}$ PstI

\section{Sense-2 long}

+1tgtgctcgcttcggcagcacatatactaacattggaacgatcctgcagGAGTAGGCGCG AGCTAAGCAGGAGGCGGAGGCGGAGGCGGAGGGCGAGGGGCGGGGAGCGCCGCCTGGAGCG CGGCAGGAAGCCTTATCAGTTGTGAGTGAGGACCAGTCGTTGTTTGAGTGTGCCTACGGAA CGCCACACCTGGCTAAGACAGAGATGACCGCGTCCTCCTCCAGCGACTATGGACAGACTTC CAAGATGAGCCCACGCGTCCCTCAGCAGGATTGGCTGTCTCAACCCCCAGCCAGGGTCACC ATCAAAATGGAATGTAACCCTAGCCAGGTGAATGGCTCAAGGAACTCTCCTGATGAATGCA GTGTGGCCAAAGGCGGGAAGATGGTGGGCAGCCCAGACACCGTTGGGATGAACTACGGCAG CTACATGGAGGAGAAGCACATGCCACCCCCAAACATGACCACGAACGAGCGCAGAGTTATC GTGCCAGCAGATCCTACGCTATGGAGTACAGACCATGTGCGGCAGTGGCTGGAGTGGGCGG TGAAAGAATATGGCCTTCCAGACGTCAACATCTTGTTATTCCAGAACATCGATGGGAAGGA ACTGTGCAAGATGACCAAGGACGACTTCCAGAGGCTCACCCCCAGCTACAACGCCGACATC CTTCTCTCACATCTCCACTACCTCAGAGAGACTCCTCTTCCACATTTGACTTCAGATGATG TTGATAAAGCCTTA

: TMPRSS2 : ERG 푸: Transcription start ctgcag: Pst I

\section{Antisense-1}

+1tcagatcgcctggagacgccatccacgctgttttgacctccatagaagacaccgggacc gatccagcctcccctcgaagctgatcctgagaacttcaggctcctgggcaacgtgctggtc tgtgtgctggcccatcactttggcaaagaat tcCTTGAGCCATTCACCTGGCTAGGGTTAC ATTCCATTTTGATGGTGACCCTGGCTGGGGGTTGAGACAGCCAATCCTGCTGAGGGACGCG TGGGCTCATCTTGGAAGTCTGTCCATAGTCGCTGGAGGAGGACGCGGTCATCTCTGTCTTA GCCAGGTGTGGCGTTCCGTAGGCACACTCAAACAACGACTGGTCCTCACTCACAACTGATA AGGCTTCCTGCCGCGCTCCAGGCGGCGCTCCCCGCCCCTCGCCCTCCGCCTCCGCCTCCGC CTCCTGCTTAGCTCGCGCCTACTCaagcttatcgataccgtcgacctcgagggcccagatc taattcaccccaccagtgcaggctgcctatcagaaagtggtggctggtgtggctaatgccc tggcccacaagtatcactaagctcgctttcttgctgtccaattctattaaaggttcctt gttccctaagtccaactactaaactgggggatattatgaagggccttccggagcatctgga ttctgcctaataaaaaacatttatttcattgcaaaaaa..........

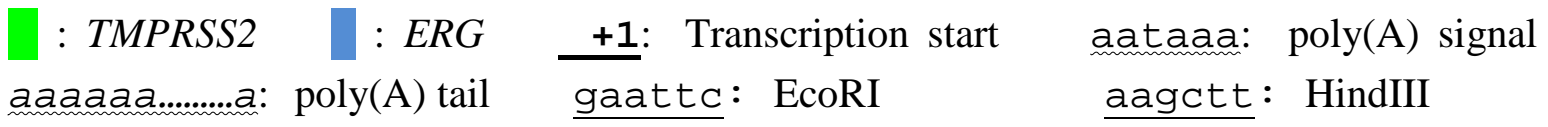




\section{Antisense-2}

+1tgtgctcgcttcggcagcacatatactaacattggaacgatcctgcagCTTGAGCCATT CACCTGGCTAGGGTTACATTCCATATTGATGGTGACCCTGGCTGGGGGTTGAGACAGCCAA TCCTGCTGAGGGACGCGTGGGCTCATCTTGGAAGTCTGTCCATAGTCGCTGGAGGAGGACG CGGTCATCTCTGTCTTAGCCAGGTGTGGCGTTCCGTAGGCACACTCAAACAACGACTGGTC CTCACTCACAACTGATAAGGCTTCCTGCCGCGCTCCAGGCGGCGCTCCCCGCCCCTCGCCC TCCGCCTCCGCCTCCGCCTCCTGCTTAGCTCGCGCCTACTC

: TMPRSS2 $\quad$ : ERG $\quad \underline{\mathbf{+ 1}}$ : Transcription start

A: $T$ to A change to inactivate the cryptic transcription termination by U6 promoter ctgcag: PstI

\section{Antisense-3}

+1tgtgctcgcttcggcagcacatatactaacattggaacgatcctgcagCTTGAGCCATT CACCTGGCTAGGGTTACATTCCATATTGATGGTGACCCTGGCTGGGGGTTGAGACAGCCAA TCCCGGACCCCGAGCCGGGACCCTGGTACCGGCGCCGCTCACCTGCCGCGCTCCAGGCGGC GCTCCCCGCCCCTCGCCCTCCGCCagacaggagtgagagatggaagctcgcgcctactC

: TMPRSS2 : ERG : Mutated region of TMPRSS2 풀 Transcription start $\underline{\text { A }}$ $\mathrm{T}$ to A change to inactivate the cryptic transcription termination by U6 promoter ctgcag: PstI

\section{Antisense-4}

+11tgtgctcgcttcggcagcacatatactaacattggaacgatcctgcagCTTGAGCCATT CACCTGGCTAGGGTTACATTCCATATTGATGGTGACCCTGGCTGGGGGTTGAGACAGCCAA TCCGACCCTGGTACCGGCGCCGCTCACCTGCCGCGCTCCAGGCGGCGCTCCCCGCCCCTCG CCCTCCGCCagacaggagtgagagatggaagctcgcgcctactc

: TMPRSS2 : ERG : Mutated region of TMPRSS2 +1: Transcription start $\underline{\mathrm{A}}$ : $\mathrm{T}$ to A change to inactivate the cryptic transcription termination by U6 promoter ctgcag: PstI

\section{Antisense-5}


+1tgtgctcgcttcggcagcacatatactaacattggaacgatcctgcagCTTGAGCCATT CACCTGGCTAGGGTTACATTCCATATTGATGGTGACCCTGGCTGGGGGTTGAGACAGCCAA TCCGCCGCTCACCTGCCGCGCTCCAGGCGGCGCTCCCCGCCCCTCGCCCTCCGCCagacag gagtgagagatggaagctcgcgcctactc

: TMPRSS2 : ERG : Mutated region of TMPRSS2 $\underline{\mathbf{+ 1}}$ : Transcription start $\underline{\mathrm{A}}$ : $\mathrm{T}$ to A change to inactivate the cryptic transcription termination by U6 promoter

ctgcag: PstI

\section{Antisense-6}

+1tgtgctcgcttcggcagcacatatactaacattggaacgatcctgcagCTTGAGCCATT CACCTGGCTAGGGTTACATTCCATATTGATGGTGACCCTGGCTGGGGGTTGAGACAGCCAA TCCCCAGGCGGCGCTCCCCGCCCCTCGCCCTCCGCCagacaggagtgagagatggaagctc gcgectactc

: TMPRSS2 : ERG : Mutated region of TMPRSS2 $\underline{\mathbf{+ 1}}$ : Transcription start $\underline{\mathrm{A}}$ : $\mathrm{T}$ to A change to inactivate the cryptic transcription termination by U6 promoter ctgcag: PstI

\section{Antisense-7}

+1tgtgctcgcttcggcagcacatatactaacattggaacgatcctgcagCTTGAGCCATT CACCTGGCTAGGGTTACATTCCATATTGATGGTGACCCTGGCTGGGGGTTGAGACAGCCAA TCCCGGACCCCGAGCCGGGACCCTGGTACCGGCGCCGCTCACCTGCCGCGCTCCAagacag gagtgagagatggaagctcgcgcctactc

: TMPRSS2 : ERG : Mutated region of TMPRSS2 풀: Transcription start $\underline{\mathrm{A}}$ : $\mathrm{T}$ to A change to inactivate the cryptic transcription termination by U6 promoter ctgcag: PstI

\section{Antisense-8}

+1tgtgctcgct tcggcagcacatatactaacat ggaacgatcctgcagCTTGAGCCATT CACCTGGCTAGGGTTACATTCCATATTGATGGTGACCCTGGCTGGGGGTTGAGACAGCCAA TCCCCAGGTTCCCCTCCCCAGCCCGGACCCCGAGCCGGGACCCTGGTACCGGCGCagacag gagtgagagatggaagctcgcgcctactc 


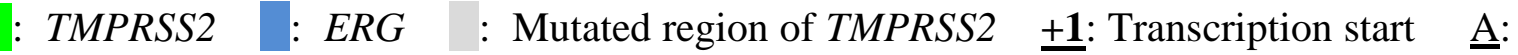
$\mathrm{T}$ to A change to inactivate the cryptic transcription termination by U6 promoter ctgcag: PstI

\section{Antisense-9}

+1tgtgctcgcttcggcagcacatatactaacattggaacgatcctgcagGAGACAGCCAA TCCTGCTGAGGGACGCGTGGGCTCATCTTGGAAGTCTGTCCATAGTCGCTGGAGGAGGACG CGGGCCGCTCACCTGCCGCGCTCCAGGCGGCGCTCCCCGCCCCTCGCCCTCCGCCagacag gagtgagagatggaagctcgcgcctactc

: TMPRSS2 : ERG : Mutated region of TMPRSS2 푸: Transcription start ctgcag: PstI

\section{Antisense-5A}

+1tgtgctcgcttcggcagcacatatactaacattggaacgatcctgcagGCCGCTCACCT GCCGCGCTCCAGGCGGCGCTCCCCGCCCCTCGCCCTCCGCCagacaggagtgagagatgga agctcgcgcctactc

: TMPRSS2 : Mutated region of TMPRSS2 $\underline{\mathbf{+ 1}}$ : Transcription start ctgcag: PstI

\section{Antisense-5B}

+1tgtgctcgcttcggcagcacatatactaacattggaacgatcctgcagCTTGAGCCATT CACCTGGCTAGGGTTACATTCCATATTGATGGTGACCCTGGCTGGGGGTTGAGACAGCCAA TCC

: ERG +1: Transcription start ctgcag: PstI $\quad \underline{\text { A: }}$ T to A change to inactivate the cryptic transcription termination by U6 promoter

\section{Antisense-B1}

+1tgtgctcgct tcggcagcacatatactaacat ggaacgatcctgcagTGGCCTGAGCC TTGAAGAATGGGGTGTACTGGGTAAATCAAAATGGTGGGGAGCATTTCCAGATGGAGAAAC TGCCTCACCTGCCGCGCTCCAGGCGGCGCTCCCCGCCCCTCGCCCTCCGCCTCCG

: TMPRSS2 : ERG 푸: Transcription start ctgcag: PstI 


\section{Antisense-B2}

+1tgtgctcgct tcggcagcacatatactaacat tggaacgatcctgcagTGGCCTGAGCC TTGAAGAATGGGGTGTACTGGGTAAATCAAAATGGTGGGGAGCATTTCCAGATGGAGAAAC TGCCCCTCCCCAGCCCGGACCCCGAGCCGGGACCCTGGTACCGGCGCCGCTCACC

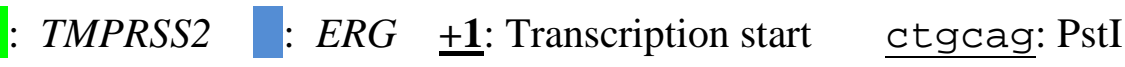

\section{Antisense-B3}

+1tgtgctcgct tcggcagcacatatactaacat tggaacgatcctgcagTGGGGAGCATT TCCAGATGGAGAAACTGCAAGGAAAAGCATAGAAGTGGGGCCACCCCTCGTGAGCTGGGGA GGGCTCACCTGCCGCGCTCCAGGCGGCGCTCCCCGCCCCTCGCCCTCCGCCTCCG

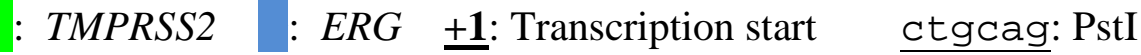

\section{Antisense-C1}

+1 tgtgctcgct tcggcagcacatatactaacat tggaacgatcctgcagTGAGCTCATGC TATTCCTATGACATAGATGAGCACTGGGTAGACCCCGTCCTGGTAACACTATTCATGCACT AACCCCAGGCGGGGGCCGTGGAGGGCAGGCGGACTAGGAGCCAGCTTTGGGGACC

: TMPRSS2 : ERG $\quad \mathbf{+ 1}:$ Transcription start cryptic transcription termination by U6 promoter
A: $T$ to A change to inactivate the ctgcag: PstI

\section{Antisense-C2}

+1tgtgctcgct tcggcagcacatatactaacat tggaacgatcctgcagTGAGCTCATGC TATTCCTATGACATAGATGAGCACTGGGTAGACCCCGTCCTGGTAACACTATTCATGCACT AACCAGCACTCCCAGTCCTCCTCCCCAAAGAGAAAAGGCGCACCGGTGCTCCCAG

: TMPRSS2 : ERG $\underline{\mathbf{+ 1}}$ : Transcription start cryptic transcription termination by U6 promoter
A: $\mathrm{T}$ to A change to inactivate the ctgcag: PstI

\section{Antisense-C3}


+1tgtgctcgcttcggcagcacatatactaacattggaacgatcctgcagGTCCTGGTAAC ACTATTCATGCACTAACAAGTTGGTTGCCAGTGAGACTTGATTATTATGACTCTGGGAGTG CTGCCCAGGCGGGGCCGTGGAGGGCAGGCGGACTAGGAGCCAGCTTTGGGGACC

: TMPRSS2 : ERG $\underline{\mathbf{+ 1}}$ : Transcription start

inactivate the cryptic transcription termination by U6 promoter
A: $T$ to A change to ctgcag: PstI

\section{Antisense-D1}

+1tgtgctcgcttcggcagcacatatactaacattggaacgatcctgcagGAGAGACAGAG AGAGAGAGGCTGGTAGAGGGAAGAGACAGAAGAAAGATGAAGGGATAAGTGTCCAGAATCC CTGAGCGCTCGACCCTCGGGCGCACTCACCTGCCGCGCCGCGCTCCTCACACCCG

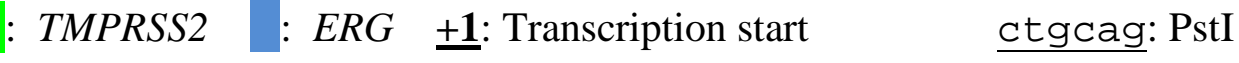

\section{Antisense-D2}

+1tgtgctcgcttcggcagcacatatactaacattggaacgatcctgcagGAGAGACAGAG AGAGAGAGGCTGGTAGAGGGAAGAGACAGAAGAAAGATGAAGGGATAAGTGTCCAGAATCC CTGCCCAGCACTCTCCCAGCACCCCGGGAGGCGCCCTGCCCGGCTGGCCCCAGCG

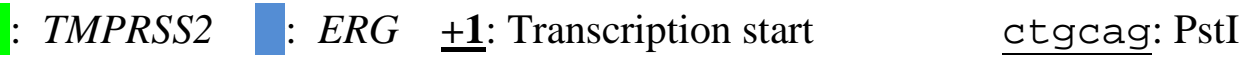

\section{Antisense-D3}

+1tgtgctcgcttcggcagcacatatactaacattggaacgatcctgcagTGAAGGGATAA GTGTCCAGAATCCCTGGATCTGGGATGGAATAAAGGATCTGGATGGTAAACGGAGAGTGCT GGGAGCGCTCGACCCTCGGGCGCACTCACCTGCCGCGCCGCGCTCCTCACACCCG

: TMPRSS2 : ERG $\underline{\mathbf{+ 1}}$ : Transcription start $\quad \underline{\text { ctgcag: PstI }}$

\section{Antisense-E1}

+1tgtgctcgcttcggcagcacatatactaacattggaacgatcctgcagAGGGTATTCAG TATTACTATTTGGCTTAGATAAGCTGGTAGTTACTTGCTAAAATTAATCTTTATTATAAAG CAGAAAGCCGCATTCTGACATCACTCTCCATGGACAAAGATTCTTCGCTTGATCA

: TMPRSS2 : ERG $\underline{\mathbf{+ 1}}$ : Transcription start the cryptic transcription termination by U6 promoter $\underline{\text { A: }}$ T to A change to inactivate ctgcag: PstI 


\section{Antisense-F1}

+1tgtgctcgcttcggcagcacatatactaacattggaacgatcctgcagAATGGATGAAT ACATAAAATAAATTGTGGTGGATATATACAACGGAATATCATTTAGCCTTTÁTTÁTTATTG AGAACTGTGCCGAGCCGGGCAGGACAGGATGAGGTGGACCGAAGCGCCCAGGTGC

TMPRSS2 : ERG $\underline{\mathbf{+ 1}}$ : Transcription start the cryptic transcription termination by U6 promoter
A: T to A change to inactivate ctgcag: PstI

\section{Antisense-G1}

+1tgtgctcgcttcggcagcacatatactaacattggaacgatcctgcagTTTATTCATGA GAAAATTCTGGCACAATGGAAACCCTGGCAAGCAAAAGATAGGGGCAGCAGATGTCCTGG CCTACAAAGAACTCCAAGCCCATCGTCCCTAGAAAGCATGGTCTCCCATGACCCC

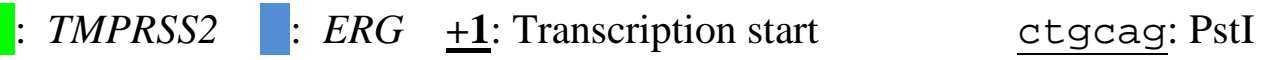

\section{Sense-3}

+1tgtgctcgcttcggcagcacatatactaacattggaacgatcctgcaggagtaggcgcg $\overline{a g} c t$ ccatctctcactcctgtctGGCGGAGGGCGAGGGGCGGGGAGCGCGCCTGGAGCG CGGCAGGTGAGCGGCGCCGGTACCAGGGTCCCGGCTCGGGGTCCGGGATTGGCTGTCTCAA CCCCAGCCAGGGTCACCATCAAAATGGAATGTAACCCTAGCCAGGTGAATGGCTCAAG

: TMPRSS2 : ERG : Mutated region of TMPRSS2 푸: Transcription start A: $\mathrm{T}$ to A change to inactivate the cryptic transcription termination by U6 promoter ctgcag: PstI

\section{Sense-4}

+1tgtgctcgcttcggcagcacatatactaacattggaacgatcctgcaggagtaggcgcg agct tccatctctcactcctgtctGGCGGAGGGCGAGGGGCGGGGAGCGCCGCCTGGAGCG CGGCAGGTGAGCGGCGCCGGTACCAGGGTCGGATTGGCTGTCTCAACCCCCAGCCAGGGTC ACCATCAATATGGAATGTAACCCTAGCCAGGTGAATGGCTCAAG

: TMPRSS2 : ERG : Mutated region of TMPRSS2 푸: Transcription start ctgcag: PstI

\section{Sense-5}


+1tgtgctcgcttcggcagcacatatactaacattggaacgatcctgcaggagtaggcgcg agcttccatctctcactcctgtctGGCGGAGGGCGAGGGGCGGGAGCGCCGCCTGGAGCG CGGCAGGTGAGCGGCGGATTGGCTGTCTCAACCCCCAGCCAGGGTCACCATCAAAATGGAA TGTAACCCTAGCCAGGTGAATGGCTCAAG

: TMPRSS2 : ERG : Mutated region of TMPRSS2 푸: Transcription start ctgcag: PstI

\section{Sense-6}

+1tgtgctcgcttcggcagcacatatactaacattggaacgatcctgcaggagtaggcgcg $\overline{a g}$ ct ccatctctcactcctgtctGGCGGAGGGCGAGGGGCGGGGAGCGCCGCCTGGGGAT TGGCTGTCTCAACCCCCAGCCAGGGTCACCATCAAAATGGAATGTAACCCTAGCCAGGTGA ATGGCTCAAG

: TMPRSS2 : ERG : Mutated region of TMPRSS2 푸: Transcription start ctgcag: PstI

\section{Sense-B1}

+1tgtgctcgcttcggcagcacatatactaacattggaacgatcctgcagCGGAGGCGGAG GGCGAGGGGCGGGGAGCGCCGCCTGGAGCGCGGCAGGTGAGGCAGTTTCTCCATCTGGAAA TGCTCCCCACCATATTGATTTACCCAGTACACCCCATTCTTCAAGGCTCAGGCCA

: TMPRSS2 : ERG $\underline{\mathbf{+ 1}}$ : Transcription start cryptic transcription termination by U6 promoter
A : T to A change to inactivate the ctgcag: PstI

\section{Sense-C1}

+1tgtgctcgct tcggcagcacatatactaacat ggaacgatcctgcagGGTCCCCAAAG CTGGCTCCTAGTCCGCCTGCCCTCCACGGCCCCCGCCTGGGGTTAGTGCATGAAGAGTGTT ACCAGGACGGGGTCTACCCAGTGCTCATCTATGTCATAGGAAGAGCATGAGCTCA

: TMPRSS2 : ERG 푸: Transcription start ctgcag: PstI

\section{Sense-D1}


+1tgtgctcgcttcggcagcacatatactaacattggaacgatcctgcagCGGGTGTGAGG AGCGCGGCGCGGCAGGTGAGTGCGCCCGAGGGTCGAGCGCTCAGGGATTCTGGACACTTAT CCCTTCATCTTTCTTCTGTCTCTTCCCTCTACCAGCCTCTCTCTCTCTGTCTCTC

: TMPRSS2 : ERG 푸: Transcription start promoter ctgcag: PstI

Antisense-TMPRSS2-ETV1-A1

+1tgtgctcget tcggcagcacatatactaacat tggaacgatcctgcagTGCCGCATTAT GTAAATCGTTCCAAGTTAAAGTCTTAGTTAGATTCAGTAGACTAGGAGCCAGCTTTGGGGA CCCCGGGGGACTCTCTTCCACCAACTGG

: TMPRSS2 : ETV1 푸: Transcription start $\quad$ ctgcag: PstI

\section{Antisense-TMPRSS2-ETV1-B1}

+1tgtgctcgcttcggcagcacatatactaacattggaacgatcctgcagGATTCAGTAAT TTCAAAGTTTATTATATTTAAGATAAGACTGAAGTGCTCAACAAACTTAGTCTCACTTTAG GTATTCCAAATGCCTTTGAACTGGGCTG

: TMPRSS2 : ETV1 푸: Transcription start $\underline{\text { A: }}$ T to A change to inactivate the cryptic transcription termination by U6 promoter ctgcag: PstI

\section{Antisense-TMPRSS2-ETV1-C1}

+1tgtgctcgcttcggcagcacatatactaacattggaacgatcctgcagCTCTGAATAGA $\overline{\text { AAAATAGAAGTCCATAGTATCAACTCTAATATTCATATTTGGCTGCATCCCCACTTCCTGG }}$ AGTACCTTCCCAGATCTCCTGGGACAGG

: TMPRSS2 : ETV1 푸: Transcription start $\quad \underline{\mathrm{A}}$ : T to A change to inactivate the cryptic transcription termination by U6 promoter ctgcag: PstI

\section{Antisense-TMPRSS2-ETV1-D1}

+1tgtgctcgcttcggcagcacatatactaacattggaacgatcctgcagATATAAACAAA

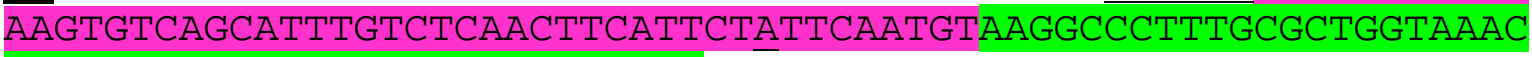
TCTCCСTGCCACACTCCСAACССССАTC 
: TMPRSS2 : ETV1 푸: Transcription start $\quad$ ctgcag: PstI

\section{Antisense-TMPRSS2-ETV1-E1}

+1tgtgctcgcttcggcagcacatatactaacattggaacgatcctgcagCTCTGAATAGA $\overline{A A A A T A G A A G T C C A T A G T A T C A A C T C T A A T A T T C A T A T T C T T C A G C A A C C A A A A C T G A A C A}$ AGCACTCCATTGACCATTCACCTTTCCT

: TMPRSS2 : ETV1 푸: Transcription start $\underline{\text { A: }}$ T to A change to inactivate the cryptic transcription termination by U6 promoter ctgcag: PstI

\section{Antisense-TMPRSS2-ETV1-F1}

+1tgtgctcgcttcggcagcacatatactaacattggaacgatcctgcagAGGAGGCCTTA AGTATGATTCAGTGAACACATTATGGTCGATAAACAAGGTGGGCCCCATTCTCAGAGTCTG ATGTAATAATTGGGACCAAGGCAATGA

: TMPRSS2 : ETV1 푸: Transcription start $\quad$ ctgcag: PstI

\section{Antisense-TMPRSS2-ETV1-G1}

+1tgtgctcgcttcggcagcacatatactaacattggaacgatcctgcagTGTAATTGACT TAGATCTTGAAAGAGTTCTAAAAAACAAGTCAAAGACATCTAGAAGAATCTCTAGATGAAG GTTACCTACAACAAAGACCAGTGTTGCC

: TMPRSS2 : ETV1 $\underline{\mathbf{+ 1}}$ : Transcription start $\quad$ ctgcag: PstI

\section{Antisense-TMPRSS2-ETV1-H1}

+1tgtgctcgcttcggcagcacatatactaacattggaacgatcctgcagTTAACAAACAG CTTAATAAATAAGCTCAGGGATACCAGAATTCACAAAAAGAAGCACTCTCCTCTGGGATCA GAGTGGGTAGGAGGATGGGGTGCAATTG

: TMPRSS2 : ETV1 푸: Transcription start $\quad$ ctgcag: PstI 


\section{Sense-TMPRSS2-ETV1-A1}

+1tgtgctcgcttcggcagcacatatactaacattggaacgatcctgcagCCAGTTGGTGG AAGAGAGTCCCCCGGGGTCCCCAAAGCTGGCTCCTAGTCTACTGAATCTAACTAAGACTTT AACTTGGAACGATTTACATAATGCGGCA

: TMPRSS2 : ETV1 푸: Transcription start $\quad$ ctgcag: PstI

\section{List of primers used}

RT-PCR primers for amplifying induced fusion RNAs:

\begin{tabular}{|c|c|}
\hline \multicolumn{2}{|c|}{ Induced fusion RNA (TMPRSS2 ex1-ERG ex7) } \\
\hline TMPRSS2 ex-1 F1 & 5’- TAGGCGCGAGCTAAGCAGGAG-3’' \\
\hline ERG ex-7 R1 & 5'- TAGCATGCATTAACCGTGGAGA-3' \\
\hline \multicolumn{2}{|c|}{ Induced fusion RNA (TMPRSS2 ex1-ERG ex4) } \\
\hline TMPRSS2 ex-1 F1 & 5’- TAGGCGCGAGCTAAGCAGGAG -3’' \\
\hline ERG ex-4 R1 & 5’-CTTGAGCCATTCACCTGGCTAG-3' \\
\hline \multicolumn{2}{|c|}{ Induced fusion RNA (TMPRSS2 ex1-ETV ex5) } \\
\hline TMPRSS2 ex-1 F1 & 5’- TAGGCGCGAGCTAAGCAGGAG-3’ \\
\hline ETV1 ex-6 R1 & 5'- TTCTTGACTGCAGGCAGAGCT -3' \\
\hline TMPRSS2 ex-1 F2 & 5’- CAGGAGGCGGAGGCGGA-3’’ \\
\hline ETV1 ex-5 R1 & 5’-CTTTCAGCCTGATAGTCTGGT-3’ \\
\hline TMPRSS2 ex-1 F3 & 5’' CGGAGGGCGAGGGGCGGGGA-3’' \\
\hline ETV1 ex-5 R2 & 5’-AACTGCTCATCATTGTCAGGT-3’ \\
\hline
\end{tabular}

Primers used in three-round PCR for amplifying induced fusion RNAs: 


\begin{tabular}{|c|c|}
\hline $1^{\text {st }}$ round PCR & \\
\hline TMPRSS2 ex-1 F1 & 5’- TAGGCGCGAGCTAAGCAGGAG -3’ \\
\hline ERG ex-4 R1 & 5’- CTTGAGCCATTCACCTGGCTAG -3’ \\
\hline $2^{\text {nd }}$ round PCR & \\
\hline TMPRSS2 ex-1 F2 & 5’- CAGGAGGCGGAGGCGGA -3’ \\
\hline ERG ex-4 R2 & 5’- TGACCCTGGCTGGGGGTTGAGA -3’ \\
\hline $3^{\text {rd }}$ round $\mathrm{PCR}$ & \\
\hline TMPRSS2 ex-1 F3 & 5’- CGGAGGGCGAGGGGCGGGGA -3’ \\
\hline ERG ex-4 R3 & 5’- TCCTGCTGAGGGACGCGTGG -3’' \\
\hline
\end{tabular}

\section{RT-PCR primers for amplifying endogenous parental mRNAs:}

\begin{tabular}{|c|c|}
\hline \multicolumn{2}{|c|}{\begin{tabular}{|l} 
TMPRSS2 parental mRNA (TMPRSS2 ex2-TMPRSS2 ex4) \\
\end{tabular}} \\
\hline TMPRSS2 ex-2 F1 & 5’- GTCATATTGAACATTCCAGA-3’ \\
\hline TMPRSS2 ex-4 R1 & 5’- GCGCAGCTCCCACGAGGAAGGT-3’ \\
\hline \multicolumn{2}{|c|}{ ERG parental mRNA (ERG ex3-ERG ex7): one-round PCR } \\
\hline$E R G$ ex-3 F1 & 5’-CAGGTTCTGAACAGCTGGTA-3’ \\
\hline ERG ex-7 R1 & 5’- TAGCATGCATTAACCGTGGAGA-3’ \\
\hline ERG ex-1 F1 & 5'- CCCCCGAGGGACATGAGAGAA-3' \\
\hline$E R G$ ex-4 R1 & 5’- TGGGGGTTGAGACAGCCAAT-3’ \\
\hline \multicolumn{2}{|c|}{ ERG parental mRNA (ERG ex3-ERG ex7): three-round PCR } \\
\hline ERG ex-3 F1 & 5’-CAGGTTCTGAACAGCTGGTA-3’ \\
\hline$E R G$ ex-7 R1 & 5'- TAGCATGCATTAACCGTGGAGA-3’ \\
\hline$E R G$ ex-3 F2 & 5’- TGGGCTGGCTTACTGAAGGA-3’ \\
\hline ERG ex-7 R2 & 5’- TTGTAAGGCTTTATCAACAT-3’ \\
\hline ERG ex-3 F3 & 5'- ATGATTCAGACTGTCCCGGA-3' \\
\hline ERG ex-7 R3 & 5’-CATCTGAAGTCAAATGTGGA-3' \\
\hline
\end{tabular}




\begin{tabular}{|l|l|}
\hline \multicolumn{2}{|l|}{$E R G$ parental mRNA (ERG ex1-ERG ex4): three-round PCR } \\
\hline$E R G$ ex-1 F1 & 5'- CCCCCGAGGGACATGAGAGAA-3' \\
\hline ERG ex-4 R1 & 5'- TGGGGGTTGAGACAGCCAAT-3’ \\
\hline ERG ex-1 F2 & 5'- AGGGACATGAGAGAAGAGGA-3' \\
\hline ERG ex-4 R2 & 5'- TGAGGGACGCGTGGGCTCAT-3' \\
\hline$E R G$ ex-1 F3 & 5'- GAGAGAAGAGGAGCGGCGCT-3' \\
\hline$E R G$ ex-4 R3 & 5'- CTTGGAAGTCTGTCCATAGT-3' \\
\hline
\end{tabular}

RT-PCR primers for amplifying BCAM-AKT2 chimeric RNA:

\section{$\mathbf{1}^{\text {st }}$ round PCR}

\begin{tabular}{|l|l|}
\hline BCAM-AKT2 F1 & 5'- GTGAGCAGCTCTCTGACCCTGA -3' \\
\hline BCAM-AKT2 R1 & 5'- CGCACCAGGATGACTTTGCCA-3' \\
\hline $\mathbf{2}^{\text {nd }}$ round PCR
\end{tabular}

\begin{tabular}{|l|l}
\hline BCAM-AKT2 F2 & 5’-TGTCTTCTACTGCGTGAGACGCA-3’ \\
\hline BCAM-AKT2 R2 & 5’- TCTCCTCAGTCGTGGAGGAGT-3’
\end{tabular}

\section{RT-PCR primers for amplifying input RNAs:}

\begin{tabular}{|c|c|}
\hline \multicolumn{2}{|l|}{ Sense-1 } \\
\hline Sense $1 \mathrm{~F} 1$ & 5'- ACGGAATTCGAGTAGGCGCGAGCTAAGCA-3' \\
\hline Sense $1 \mathrm{R} 1$ & 5’- TAGAAGCTTCTTGAGCCATTCACCTGGCT-3’ \\
\hline \multicolumn{2}{|l|}{ Sense-2 } \\
\hline Sense 2 F1 & 5’- ACGCTGCAGGAGTAGGCGCGAGCTAAGCA-3’' \\
\hline Sense 2 R1 & 5’- TAGAAGCTTAAAAAACTTGAGCCATTCACCTGGCT-3’ \\
\hline \multicolumn{2}{|l|}{ Sense-2 long } \\
\hline Sense 2 long F1 & 5’- ACGCTGCAGGAGTAGGCGCGAGCTAAGCA-3’' \\
\hline
\end{tabular}


bioRxiv preprint doi: https://doi.org/10.1101/151241; this version posted June 16, 2017. The copyright holder for this preprint (which was not certified by peer review) is the author/funder, who has granted bioRxiv a license to display the preprint in perpetuity. It is made available under aCC-BY 4.0 International license.

\begin{tabular}{|c|c|}
\hline Sense 2 long R1 & 5’- TAGAAGCTTAAAAAATAAGGCTTTATCAACATCAT-3' \\
\hline \multicolumn{2}{|l|}{ Antisense-1 } \\
\hline Antisense $1 \mathrm{~F} 1$ & 5’- ACGGAATTCCTTGAGCCATTCACCTGGCT-3’ \\
\hline Antisense $1 \mathrm{R} 1$ & 5’- TAGAAGCTTGAGTAGGCGCGAGCTAAGCA-3’' \\
\hline \multicolumn{2}{|l|}{ Antisense-2 } \\
\hline Antisense 2 F1 & 5’- ACGCTGCAGCTTGAGCCATTCACCTGGCTAGGGTT-3’ \\
\hline Antisense 2 R1 & 5’- TAGAAGCTTAAAAAAGAGTAGGCGCGAGCTAAGCA-3’ \\
\hline \multicolumn{2}{|l|}{ Antisense-3 } \\
\hline Antisense $3 \mathrm{~F} 1$ & 5’- ACGCTGCAGCTTGAGCCATTCACCTGGCTAGGGTT-3’’ \\
\hline Antisense $3 \mathrm{R} 1$ & 5'- TAGAAGCTTAAAAAAGAGTAGGCGCGAGCTTCCAT-3’ \\
\hline \multicolumn{2}{|l|}{ Antisense-4 } \\
\hline Antisense 4 F1 & 5’- ACGCTGCAGCTTGAGCCATTCACCTGGCTAGGGTT-3’ \\
\hline Antisense 4 R1 & 5’- TAGAAGCTTAAAAAAGAGTAGGCGCGAGCTTCCAT-3’ \\
\hline \multicolumn{2}{|l|}{ Antisense-5 } \\
\hline Antisense 5 F1 & 5’- ACGCTGCAGCTTGAGCCATTCACCTGGCTAGGGTT-3’ \\
\hline Antisense 5 R1 & 5'- TAGAAGCTTAAAAAAGAGTAGGCGCGAGCTTCCAT-3’ \\
\hline \multicolumn{2}{|l|}{ Antisense-6 } \\
\hline Antisense 6 F1 & 5’- ACGCTGCAGCTTGAGCCATTCACCTGGCTAGGGTT-3’ \\
\hline Antisense 6 R1 & 5’- TAGAAGCTTAAAAAAGAGTAGGCGCGAGCTTCCAT-3’ \\
\hline \multicolumn{2}{|l|}{ Antisense-7 } \\
\hline Antisense 7 F1 & 5’- ACGCTGCAGCTTGAGCCATTCACCTGGCTAGGGTT-3’’ \\
\hline Antisense $7 \mathrm{R} 1$ & 5’- TAGAAGCTTAAAAAAGAGTAGGCGCGAGCTTCCAT-3’ \\
\hline \multicolumn{2}{|l|}{ Antisense-8 } \\
\hline Antisense $8 \mathrm{~F} 1$ & 5’- ACGCTGCAGCTTGAGCCATTCACCTGGCTAGGGTT-3’ \\
\hline Antisense $8 \mathrm{R} 1$ & 5’- TAGAAGCTTAAAAAAGAGTAGGCGCGAGCTTCCAT-3’ \\
\hline Antisense-9 & \\
\hline
\end{tabular}


bioRxiv preprint doi: https://doi.org/10.1101/151241; this version posted June 16, 2017. The copyright holder for this preprint (which was not certified by peer review) is the author/funder, who has granted bioRxiv a license to display the preprint in perpetuity. It is made available under aCC-BY 4.0 International license.

\begin{tabular}{|c|c|}
\hline Antisense 9 F1 & 5’- ACGCTGCAGGAGACAGCCAATCCTGCTGAGGGACGCGTGGGC-3’ \\
\hline Antisense 9 R1 & 5’- TAGAAGCTTAAAAAAGAGTAGGCGCGAGCTTCCAT-3’ \\
\hline \multicolumn{2}{|l|}{ Antisense-5A } \\
\hline Antisense 5A F1 & 5’ - TAGAAGCTTAAAAAAGAGTAGGCGCGAGCTTCCAT-3’’ \\
\hline Antisense 5A R1 & 5’-TAGCTGCAGGCCGCTCACCTGCCGCGCTCC-3’’ \\
\hline \multicolumn{2}{|l|}{ Antisense-5B } \\
\hline Antisense 5B F1 & 5’- ACGAAGCTTAAAAAAGGATTGGCTGTCTCAACCCCCA-3’’ \\
\hline Antisense 5B R1 & 5’- ACGCTGCAGCTTGAGCCATTCACCTGGCTAGGGTT-3’’ \\
\hline \multicolumn{2}{|l|}{ Antisense-B1 } \\
\hline Antisense B1 F1 & 5’-TAGCTGCAGTGGCCTGAGCCTTGAAGAAT-3’ \\
\hline Antisense B1 R1 & 5’-ACGAAGCTTAAAAAACGGAGGCGGAGGGCGAGGGG-3’ \\
\hline \multicolumn{2}{|l|}{ Antisense-B2 } \\
\hline Antisense B2 F1 & 5’- TAGCTGCAGTGGCCTGAGCCTTGAAGAAT-3’ \\
\hline Antisense B2 R1 & 5’- ACGAAGCTTAAAAAAGGTGAGCGGCGCCGGTAC-3’ \\
\hline \multicolumn{2}{|l|}{ Antisense-B3 } \\
\hline Antisense B3 F1 & 5’- TAGCTGCAGTGGGGAGCATTTCCAGATGGAGAAACTGCAAGG-3’ \\
\hline Antisense B3 R1 & 5’- ACGAAGCTTAAAAAACGGAGGCGGAGGGCGAGGG-3’ \\
\hline \multicolumn{2}{|l|}{ Antisense-C1 } \\
\hline Antisense C1 F1 & 5’- TAGCTGCAGTGAGCTCATGCTATTCCTATGA-3’’ \\
\hline Antisense C1 R1 & 5’- ACGAAGCTTAAAAAAGGTCCCCAAAGCTGGCTCCT-3’’ \\
\hline \multicolumn{2}{|l|}{ Antisense-C2 } \\
\hline Antisense C2 F1 & 5’- TAGCTGCAGTGAGCTCATGCTATTCCTATGA -3’ \\
\hline Antisense C2 R1 & 5’- ACGAAGCTTAAAAAACTGGGAGCACCGGTGCGC-3’ \\
\hline \multicolumn{2}{|l|}{ Antisense-C3 } \\
\hline Antisense C3 F1 & 5’- TAGCTGCAGGTCCTGGTAACACTATTCATGCACTAACAAGTT-3’ \\
\hline Antisense C3 R1 & 5’- ACGAAGCTTAAAAAAGGTCCCCAAAGCTGGCTCCT-3’ \\
\hline
\end{tabular}


bioRxiv preprint doi: https://doi.org/10.1101/151241; this version posted June 16, 2017. The copyright holder for this preprint (which was not certified by peer review) is the author/funder, who has granted bioRxiv a license to display the preprint in perpetuity. It is made available under aCC-BY 4.0 International license.

\begin{tabular}{|c|c|}
\hline \multicolumn{2}{|l|}{ Antisense-D1 } \\
\hline Antisense D1 F1 & 5’-TAGCTGCAGGAGAGACAGAGAGAGAGAGG-3’' \\
\hline Antisense D1 R1 & 5’- ACGAAGCTTAAAAAACGGGTGTGAGGAGCGCGGCG-3' \\
\hline \multicolumn{2}{|l|}{ Antisense-D2 } \\
\hline Antisense D2 F1 & 5’- TAGCTGCAGGAGAGACAGAGAGAGAGAGG-3' \\
\hline Antisense D2 R1 & 5’- ACGAAGCTTAAAAAACGCTGGGGCCAGCCGGGC-3’' \\
\hline \multicolumn{2}{|l|}{ Antisense-D3 } \\
\hline Antisense D3 F1 & 5’- TAGCTGCAGTGAAGGGATAAGTGTCCAGAATCCCTGGATCTG-3’ \\
\hline Antisense D3 R1 & 5’- ACGAAGCTTAAAAAACGGGTGTGAGGAGCGCGGCG-3’ \\
\hline \multicolumn{2}{|l|}{ Antisense-E1 } \\
\hline Antisense E1 F1 & 5’- TAGCTGCAGAGGGTATTCAGTATTACTATTT-3’’ \\
\hline Antisense E1 R1 & 5’- ACGAAGCTTAAAAAATGATCAAGCGAAGAATCTTTGTCCATG-3’ \\
\hline \multicolumn{2}{|l|}{ Antisense-F1 } \\
\hline Antisense F1 F1 & 5’- TAGCTGCAGAATGGATGAATACATAAAATAA-3’ \\
\hline Antisense F1 R1 & 5’- ACGAAGCTTAAAAAAGCACCTGGGCGCTTCGGTCCACCTCAT-3’ \\
\hline \multicolumn{2}{|l|}{ Antisense-G1 } \\
\hline Antisense G1 F1 & 5’- TAGCTGCAGTTTATTCATGAGAAAATTCTGG-3’ \\
\hline Antisense G1 R1 & 5’- ACGAAGCTTAAAAAAGGGGTCATGGGAGACCATGCTTTCTAG-3’ \\
\hline \multicolumn{2}{|l|}{ Sense-3 } \\
\hline Sense $3 \mathrm{~F} 1$ & 5’- ACGCTGCAGGAGTAGGCGCGAGCTTCCAT-3’’ \\
\hline Sense 3 R1 & 5’- TAGAAGCTTAAAAAACTTGAGCCATTCACCTGGCT-3’ \\
\hline \multicolumn{2}{|l|}{ Sense-4 } \\
\hline Sense $4 \mathrm{~F} 1$ & 5’- ACGCTGCAGGAGTAGGCGCGAGCTTCCAT-3’’ \\
\hline Sense $4 \mathrm{R} 1$ & 5’- TAGAAGCTTAAAAAACTTGAGCCATTCACCTGGCT-3’ \\
\hline \multicolumn{2}{|l|}{ Sense-5 } \\
\hline Sense 5 F1 & 5’- ACGCTGCAGGAGTAGGCGCGAGCTTCCAT-3’’ \\
\hline
\end{tabular}


bioRxiv preprint doi: https://doi.org/10.1101/151241; this version posted June 16, 2017. The copyright holder for this preprint (which was not certified by peer review) is the author/funder, who has granted bioRxiv a license to display the preprint in perpetuity. It is made available under aCC-BY 4.0 International license.

\begin{tabular}{|c|c|}
\hline Sense $5 \mathrm{R} 1$ & 5’- TAGAAGCTTAAAAAACTTGAGCCATTCACCTGGCT-3’’ \\
\hline \multicolumn{2}{|l|}{ Sense-6 } \\
\hline Sense 6 F1 & 5’- ACGCTGCAGGAGTAGGCGCGAGCTTCCAT-3’’ \\
\hline Sense 6 R1 & 5’- TAGAAGCTTAAAAAACTTGAGCCATTCACCTGGCT-3’' \\
\hline \multicolumn{2}{|l|}{ Sense-B1 } \\
\hline Sense B1 F1 & 5’- TAGCTGCAGCGGAGGCGGAGGGCGAGGGG-3’ \\
\hline Sense B1 R1 & 5’- ACGAAGCTTAAAAAATGGCCTGAGCCTTGAAGAAT-3’ \\
\hline \multicolumn{2}{|l|}{ Sense-C1 } \\
\hline Sense C1 F1 & 5’-TAGCTGCAGGGTCCCCAAAGCTGGCTCCT-3’ \\
\hline Sense C1 R1 & 5’- ACGAAGCTTAAAAAATGAGCTCATGCTCTTCCTAT-3’’ \\
\hline \multicolumn{2}{|l|}{ Sense-D1 } \\
\hline Sense D1 F1 & 5’- TAGCTGCAGCGGGTGTGAGGAGCGCGGCG-3’' \\
\hline Sense D1 R1 & 5’- ACGAAGCTTAAAAAAGAGAGACAGAGAGAGAGAGG-3’ \\
\hline \multicolumn{2}{|c|}{ Antisense TMPRSS2-ETV1-A1 } \\
\hline $\begin{array}{l}\text { Antisense } \\
\text { TMPRSS2- } \\
\text { ETV1-A1 F1 }\end{array}$ & 5’- TAGCTGCAGTGCCGCATTATGTAAATCGTTCCAAGTTAAAGTC-3’ \\
\hline $\begin{array}{l}\text { Antisense } \\
\text { TMPRSS2- } \\
\text { ETV1-A1 R1 }\end{array}$ & $\begin{array}{l}\text { 5'- ACGAAGCTTAAAAAACCAGTTGGTGGAAGAGAGTCCCCCGGG- } \\
\text { 3' }\end{array}$ \\
\hline \multicolumn{2}{|c|}{ Antisense TMPRSS2-ETV1-B1 } \\
\hline $\begin{array}{l}\text { Antisense } \\
\text { TMPRSS2- } \\
\text { ETV1-B1 F1 }\end{array}$ & 5’- TAGCTGCAGGATTCAGTAATTTCAAAGTTTATTATATTTAAGA-3’ \\
\hline $\begin{array}{l}\text { Antisense } \\
\text { TMPRSS2- } \\
\text { ETV1-B1 R1 }\end{array}$ & 5’- ACGAAGCTTAAAAAACAGCCCAGTTACAAGGCATTTGGAATA-3’ \\
\hline \multicolumn{2}{|c|}{ Antisense TMPRSS2-ETV1-C1 } \\
\hline $\begin{array}{l}\text { Antisense } \\
\text { TMPRSS2- }\end{array}$ & 5’- TAGCTGCAGCTCTGAATAGAAAAATAGAAGTCCATAGTATCAA- \\
\hline
\end{tabular}


bioRxiv preprint doi: https://doi.org/10.1101/151241; this version posted June 16, 2017. The copyright holder for this preprint (which was not certified by peer review) is the author/funder, who has granted bioRxiv a license to display the preprint in perpetuity. It is made available under aCC-BY 4.0 International license.

\begin{tabular}{|c|c|}
\hline ETV1-C1 F1 & 3’ \\
\hline $\begin{array}{l}\text { Antisense } \\
\text { TMPRSS2- } \\
\text { ETV1-C1 R1 }\end{array}$ & 5’- ACGAAGCTTAAAAAACCTGTCCCAGGAGATCTGGGAAGGTAC-3’ \\
\hline \multicolumn{2}{|c|}{ Antisense TMPRSS2-ETV1-D1 } \\
\hline $\begin{array}{l}\text { Antisense } \\
\text { TMPRSS2- } \\
\text { ETV1-D1 F1 }\end{array}$ & $\begin{array}{l}\text { 5’- TAGCTGCAGATATAAACAAAAAGTGTCAGCATTTGTCTCAACT- } \\
\text { 3' }\end{array}$ \\
\hline $\begin{array}{l}\text { Antisense } \\
\text { TMPRSS2- } \\
\text { ETV1-D1 R1 }\end{array}$ & $\begin{array}{l}\text { 5’- ACGAAGCTTAAAAAAGATGGGGGTTGGGAGTGTGGCAGGGAG- } \\
\text { 3' }\end{array}$ \\
\hline \multicolumn{2}{|c|}{ Antisense TMPRSS2-ETV1-E1 } \\
\hline $\begin{array}{l}\text { Antisense } \\
\text { TMPRSS2- } \\
\text { ETV1-E1 F1 }\end{array}$ & $\begin{array}{l}\text { 5’- TAGCTGCAGCTCTGAATAGAAAAATAGAAGTCCATAGTATCAA- } \\
\text { 3’ }\end{array}$ \\
\hline $\begin{array}{l}\text { Antisense } \\
\text { TMPRSS2- } \\
\text { ETV1-E1 R1 }\end{array}$ & $\begin{array}{l}\text { 5’- ACGAAGCTTAAAAAAAGGAAAGGTGAATGGTCAATGGAGTGC- } \\
\text { 3’ }\end{array}$ \\
\hline \multicolumn{2}{|c|}{ Antisense TMPRSS2-ETV1-F1 } \\
\hline $\begin{array}{l}\text { Antisense } \\
\text { TMPRSS2- } \\
\text { ETV1-F1 F1 }\end{array}$ & $\begin{array}{l}\text { 5’- TAGCTGCAGAGGAGGCCTTAAGTATGATTCAGTGAACACATTA- } \\
\text { 3' }\end{array}$ \\
\hline $\begin{array}{l}\text { Antisense } \\
\text { TMPRSS2- } \\
\text { ETV1-F1 R1 }\end{array}$ & 5’- ACGAAGCTTAAAAAAATCATTGCCTTGGTCCCAATTATTACA-3’ \\
\hline \multicolumn{2}{|c|}{ Antisense TMPRSS2-ETV1-G1 } \\
\hline $\begin{array}{l}\text { Antisense } \\
\text { TMPRSS2- } \\
\text { ETV1-G1 F1 }\end{array}$ & $\begin{array}{l}\text { 5’- TAGCTGCAGTGTAATTGACTTAGATCTTGAAAGAGTTCTAAAA- } \\
\text { 3’ }\end{array}$ \\
\hline $\begin{array}{l}\text { Antisense } \\
\text { TMPRSS2- } \\
\text { ETV1-G1 R1 }\end{array}$ & 5’- ACGAAGCTTAAAAAAGGCAACACTGGTCTTTGTTGTAGGTAA-3’ \\
\hline Antisense $T M$ & 2-ETV1-H1 \\
\hline
\end{tabular}




\begin{tabular}{|c|c|}
\hline $\begin{array}{l}\text { Antisense } \\
\text { TMPRSS2- } \\
\text { ETV1-H1 F1 }\end{array}$ & $\begin{array}{l}\text { 5’- TAGCTGCAGTTAACAAACAGCTTAATAAATAAGCTCAGGGATA- } \\
\text { 3' }\end{array}$ \\
\hline $\begin{array}{l}\text { Antisense } \\
\text { TMPRSS2- } \\
\text { ETV1-H1 R1 }\end{array}$ & 5’- ACGAAGCTTAAAAAACAATTGCACCССАТССТCСТАСССАСТ-3’ \\
\hline \multicolumn{2}{|c|}{ Sense TMPRSS2-ETV1-A1 } \\
\hline $\begin{array}{l}\text { Sense } \\
\text { TMPRSS2- } \\
\text { ETV1-A1 F1 }\end{array}$ & 5’- TAGCTGCAGCCAGTTGGTGGAAGAGAGT-3’ \\
\hline $\begin{array}{l}\text { Sense } \\
\text { TMPRSS2- } \\
\text { ETV1-A1 R1 }\end{array}$ & 5’- ACGAAGCTTAAAAAATGCCGCATTATGTAAATC-3’ \\
\hline
\end{tabular}

PCR primers used for amplifying the identified TMPRSS2-ERG genomic DNA breakpoint:

\begin{tabular}{|l|l|}
\hline \multicolumn{2}{|l|}{ TMPRSS2 intron 1} \\
\hline TMPRSS2 genomic bk F1 & 5'- ATGTGATATTAGTGCGGTTA -3' \\
\hline TMPRSS2 genomic bk F2 & 5'- GGCTGGGATGTGTCCGTGGA -3' \\
\hline ERG intron 3 & 5'- CCATAAGTTTTACTGCGTCT-3' \\
\hline ERG genomic bk R1 & 5'- GATACTGAGTGGTAAATTCT-3' \\
\hline ERG genomic bk R2 &
\end{tabular}

The rest of PCR primers used for genomic breakpoint analyses are not listed, but their locations are shown in Fig. S14A.

PCR primers used for amplifying the identified TMPRSS2-ETV1 genomic DNA breakpoint:

\section{TMPRSS2}

TMPRSS2 genomic bk pt A1

5’ - TCTGCTCGAGCACGGGTCCA-3’ 


\begin{tabular}{|l|l|}
\hline TMPRSS2 genomic bk pt A2 & 5’-AAAACTGCCCCATGTCCAG-3' \\
\hline TMPRSS2 genomic bk pt B1 & 5'- CAACCTGGGAGGCCCTGCCT-3' \\
\hline TMPRSS2 genomic bk pt B2 & 5'- CAGCAACAGCACAAGCTTGT-3' \\
\hline TMPRSS2 genomic bk pt C1 & 5'- GGCTGGGATGTGTCCGTGGA-3' \\
\hline TMPRSS2 genomic bk pt C2 & 5'- TGGTGGTGGTGCTGTCTGGA-3' \\
\hline TMPRSS2 genomic bk pt D1 & 5'- CAGGAGAATCACTTGAACCT-3' \\
\hline TMPRSS2 genomic bk pt D2 & 5'- AGGCCACTGCACTCCAGCCT-3' \\
\hline ERG intron 3 & 5'-GCAAGTCTCGTTGATCGCCA-3' \\
\hline ETV1 genomic bk pt M1 & 5'-TTGCACACGTTTGCGAATCA-3' \\
\hline ETV1 genomic bk pt M2 & 5'-AGGGAGAGTTGCTTCCCAGT-3' \\
\hline ETV1 genomic bk pt N1 & 5'-GCCGATCTTAGCACATTACT-3' \\
\hline ETV1 genomic bk pt N2 &
\end{tabular}

\section{References for Supplementary Information}

Kimsey, I., and Al-Hashimi, H.M. (2014). Increasing occurrences and functional roles for high energy purine-pyrimidine base-pairs in nucleic acids. Curr Opin Struct Biol 24, 72-80.

Mertz, K.D., Setlur, S.R., Dhanasekaran, S.M., Demichelis, F., Perner, S., Tomlins, S., Tchinda, J., Laxman, B., Vessella, R.L., Beroukhim, R., et al. (2007). Molecular characterization of TMPRSS2-ERG gene fusion in the NCI-H660 prostate cancer cell line: a new perspective for an old model. Neoplasia 9, 200-206.

Rychlik, W., and Rhoads, R.E. (1989). A computer program for choosing optimal oligonucleotides for filter hybridization, sequencing and in vitro amplification of DNA. Nucleic Acids Res 17, 8543-8551.

Teles Alves, I., Hiltemann, S., Hartjes, T., van der Spek, P., Stubbs, A., Trapman, J., and Jenster, G. (2013). Gene fusions by chromothripsis of chromosome $5 q$ in the VCaP prostate cancer cell line. Hum Genet 132, 709-713.

Watson, J.D., and Crick, F.H. (1953). Genetical implications of the structure of deoxyribonucleic acid. Nature 171, 964-967.

Weier, C., Haffner, M.C., Mosbruger, T., Esopi, D.M., Hicks, J., Zheng, Q., Fedor, H., Isaacs, W.B., De Marzo, A.M., Nelson, W.G., et al. Nucleotide resolution analysis of TMPRSS2 and ERG rearrangements in prostate cancer. J Pathol 230, 174-183. 